TRANSACTIONS OF THE

AMERICAN MATHEMATICAL SOCIETY

Volume 348, Number 7, July 1996

\title{
PROPAGATION OF GEVREY REGULARITY FOR A CLASS OF HYPOELLIPTIC EQUATIONS
}

\author{
ANTONIO BOVE AND DAVID S. TARTAKOFF
}

\begin{abstract}
We prove results on the propagation of Gevrey and analytic wave front sets for a class of $C^{\infty}$ hypoelliptic equations with double characteristics.
\end{abstract}

\section{INTRODUCTION}

It is well known that a (pseudo-differential) operator with semi-definite principal part and at most double characteristics is not, in general, $C^{\infty}$ hypoelliptic; however if the lower order terms satisfy some supplementary assumptions then there is $C^{\infty}$ hypoellipticity (see e.g. [7], vol. III, [6]).

As far as analytic hypoellipticity is concerned, the situation is more involved. There are in fact examples of operators being $C^{\infty}$ hypoelliptic (i.e. whose lower order terms satisfy the "Levi" conditions) but not analytic hypoelliptic. At the same time, it has been proved that if the operator's principal part vanishes exactly of order 2 on a manifold $\Sigma$ in the cotangent bundle, if its lower order terms satisfy the $C^{\infty}$ hypoellipticity conditions and if $\Sigma$ is symplectic (i.e. the symplectic form $\sigma=d \xi \wedge d x$ has maximal rank on $T \Sigma$ ) then there is analytic hypoellipticity (see e.g. [10], [14], [16], [15]).

The situation becomes more involved if the symplectic form does not have maximal rank or if it can degenerate on a submanifold (subset) of the double characteristic manifold. It has been proved by Métivier ([12], [9]) that actually there is propagation of the analytic singularities on the leaves of the characteristic manifold if the operator satisfies the conditions for $C^{\infty}$ hypoellipticity with loss of one derivative. Essentially, Métivier constructs microlocal null solutions for certain microlocal models having a non empty analytic wave front set. Moreover, analyzing the same microlocal model, he proved a theorem of propagation of the analytic regularity.

A much deeper analysis has been carried out by Sjöstrand, [13], using F.B.I.S. transform with Lipschitz Lagrangian manifold, considering also the case in which the rank of the fundamental matrix of the principal symbol may degenerate on a submanifold of the double characteristic manifold. In particular he gave another proof of Métivier's theorem on the propagation of the regularity.

The purpose of the present paper is to give another proof of those microlocal hypoellipticity results for operators with double characteristics satisfying the conditions for $C^{\infty}$ hypoellipticity with loss of one derivative. A first result essentially states that the Gevrey wave front set $W F_{s}$ (or rather its complement, i.e. the set

Received by the editors November 21, 1994

1991 Mathematics Subject Classification. Primary 35H05, 35G05, 35D10, 46F99.

(C)1996 American Mathematical Society 
of points of $G^{s}$-regularity), $s \geq 1$, propagates along the leaves of the characteristic manifold $\Sigma$ (in particular for symplectic manifolds we get analytic hypoellipticity). The second result says that an operator in the above mentioned class is actually $G^{s}$ hypoelliptic if $s \geq 2$. This second result, using different techniques, has been obtained by Kajitani-Wakabayashi in [8].

Our technique is to deduce a priori $G^{s}$ bounds using as a starting point the a priori hypoellipticity estimates in the $C^{\infty}$ case. To do this we need a careful microlocalization procedure in the directions tangent to $\Sigma$. This is accomplished by a technique due to the second author, [14], [15], and already used to give an alternative proof of Métivier's theorem [10], although the full details are still unpublished. We feel that this technique can be useful in more generality and in degenerate situations.

The first five sections of the paper are devoted to establishing the notation, introducing the microlocalization and proving the first theorem. The sixth section is concerned with the proof of the second theorem. An appendix collects some general-purpose material used throughout the paper.

Finally the first author would like to take this opportunity to thank the Department of Mathematics of the University of Illinois at Chicago, where he stayed for three weeks during the preparation of the final version of this paper. This allowed him to enjoy lots of mathematics and to short cut the clumsiness of e-mail!

\section{Preparations and Statement of Results}

Let $P\left(x, D_{x}\right)=P_{m}\left(x, D_{x}\right)+P_{m-1}\left(x, D_{x}\right)+\cdots$ be a classical $G^{s}$ (pseudo)differential operator of order $m, s \geq 1$, and denote by $p_{m-j}$ the symbols of the $P_{m-j}$, which are (positively) homogeneous of degree $m-j$ with respect to $\xi$. We shall make the following assumptions:

$\left(\mathbf{H}_{1}\right): \quad(\mathbf{a}): p_{m}(x, \xi) \geq 0$, for every $(x, \xi) \in T^{*} \mathbb{R}^{n} \backslash\{0\}$.

(b): Let $\Sigma=\left\{p_{m}(x, \xi)=0\right\}$. Then $\Sigma$ is a real $G^{s}$ manifold in $T^{*} \mathbb{R}^{n} \backslash\{0\}$.

(c): $p_{m}$ vanishes on $\Sigma$ exactly of order 2, i.e. $p_{m}(x, \xi) \geq$ Const $d_{\Sigma}^{2}(x, \xi)$, where $d_{\Sigma}(x, \xi)$ denotes the distance of the point $(x, \xi) \in T^{*} \mathbb{R}^{n} \backslash\{0\}$ from $\Sigma$.

(d): Let $F_{p_{m}}\left(x_{0}, \xi_{0}\right)$ denote the Hamilton map of $p_{m}$ at $\left(x_{0}, \xi_{0}\right) \in \Sigma$, defined by

$$
\left\langle F_{p_{m}}\left(x_{0}, \xi_{0}\right) t, d \varphi\right\rangle=\frac{1}{2}\left\langle t, d\left(H_{p_{m}} \varphi\right)\left(x_{0}, \xi_{0}\right)\right\rangle,
$$

where $t \in T_{\left(x_{0}, \xi_{0}\right)}\left(T^{*} \mathbb{R}^{n} \backslash\{0\}\right)$ and $\varphi$ is a smooth function. Then

$$
\operatorname{dim} \operatorname{ker} F_{p_{m}}\left(x_{0}, \xi_{0}\right)=\operatorname{dim} T_{\left(x_{0}, \xi_{0}\right)}^{\Sigma}
$$

and

$$
\sigma_{\mid \Sigma} \quad \text { has constant rank. }
$$

Without any loss of generality we shall suppose henceforth that $m=2$, the general case being recovered multiplying by an elliptic pseudo-differential factor.

Denote by $p_{1}^{s}(x, \xi)$ the subprincipal symbol of $P$, defined by

$$
p_{1}^{s}(x, \xi)=p_{1}(x, \xi)+\frac{i}{2} \sum_{j=1}^{n} \frac{\partial^{2} p_{2}(x, \xi)}{\partial x_{j} \partial \xi_{j}} ;
$$


it is invariantly defined at points $(x, \xi)$ belonging to $\Sigma$. Furthermore we shall denote by

$$
\operatorname{Tr}^{+} F_{p_{m}}(x, \xi)=\sum_{\substack{i \mu \in \operatorname{sp} \\ \mu>0}} \mu .
$$

Since we are interested in micolocal results we shall always work in a microlocal neighborhood, $U$, of $\left(x_{0}, \xi_{0}\right) \in \Sigma$.

We make the following assumption on the lower order terms:

$\left(\mathbf{H}_{2}\right)$ : If $\left(x_{0}, \xi_{0}\right) \in \Sigma$, then $p_{1}^{s}\left(x_{0}, \xi_{0}\right)+\operatorname{Tr}^{+} F_{p_{2}}\left(x_{0}, \xi_{0}\right) \notin \overline{\mathbb{R}^{-}}$.

Because of $\left(\mathrm{H}_{1}\right)$ and $\left(\mathrm{H}_{2}\right)$ we can find a canonical $G^{s}$ transformation, $\Phi$, defined in $U$, such that

$$
\begin{gathered}
\Phi\left(x_{0}, \xi_{0}\right)=\left(0, e_{n}\right) \\
\text { if }(y, \eta)=\Phi(x, \xi), \quad y=\left(y^{\prime}, y^{\prime \prime}, y^{\prime \prime \prime}\right) \in \mathbb{R}^{k+\ell+n-k-\ell},
\end{gathered}
$$

$2 k$ being the rank of $\sigma_{\mid \Sigma}, \ell=\operatorname{dim} \operatorname{Im} F_{p_{2}}(x, \xi)-2 k$.

Moreover in the coordinates $(y, \eta), P$ can be written as

$$
\begin{gathered}
\sum_{i, j=1}^{k} a_{i j}(y, \eta) X_{i} X_{j}^{*}+\sum_{j=1}^{k} \sum_{s=1}^{\ell}\left(b_{j s}(y, \eta) X_{j} Y_{s}+b_{j s}^{*}(y, \eta) X_{j}^{*} Y_{s}\right) \\
+\sum_{r, s=1}^{\ell} c_{r s}(y, \eta) Y_{r} Y_{s}+\tilde{p}_{1}(y, \eta)+\sum_{j=1}^{k} \alpha_{j}^{\prime}(y, \eta) X_{j}+\sum_{j=1}^{k} \alpha_{j}^{\prime \prime}(y, \eta) X_{j}^{*} \\
+\sum_{s=1}^{\ell} \beta_{s}(y, \eta) Y_{s}+\tilde{p}_{0}(y, \eta)
\end{gathered}
$$

where, denoting by $A(y, \eta)=\left[a_{i j}(y, \eta)\right]_{i, j=1, \ldots, k}, C(y, \eta)=\left[c_{r s}(y, \eta)\right]_{r, s=1, \ldots, \ell}$, and $B(y, \eta)=\left[b_{j s}(y, \eta)\right]_{\substack{j=1, \ldots, k \\ s=1, \ldots, \ell}}$, the matrix

$$
\left[\begin{array}{cc}
A & B \\
B^{*} & C
\end{array}\right]
$$

is a self-adjoint, positive definite matrix of pseudo-differential operators of order $0 ; \tilde{p}_{1}(y, \eta)$ is a first-order pseudo-differential operator such that $\tilde{p}_{1}(y, \eta)_{\mid \Sigma}=$ $\left(p_{1}^{s}(x, \xi)+\operatorname{Tr}^{+} F_{p_{2}}\right)_{\mid \Sigma} ; \alpha_{j}^{\prime}, \alpha_{j}^{\prime \prime}, j=1, \cdots, k, \beta_{s}, s=1, \cdots, \ell$, and $\tilde{p}_{0}$ are pseudodifferential operators of order 0 . Moreover

$$
\sqrt{2} X=D_{y^{\prime}}-i y^{\prime}\left|D_{y_{n}}\right|, \quad Y=D_{y^{\prime \prime}}
$$

Using Métivier's technique of addition of variables and making another analytic canonical transformation we can write the operator in (2.3) as

$$
\left\langle\mathcal{A}(x, \xi)\left[\begin{array}{c}
X \\
Y
\end{array}\right],\left[\begin{array}{c}
X^{*} \\
Y
\end{array}\right]\right\rangle+\left\langle L(x, \xi),\left[\begin{array}{c}
X^{*} \\
Y
\end{array}\right]\right\rangle+\tilde{p}_{1}(x, \xi)+\tilde{p}_{0}(x, \xi),
$$

where

$$
\begin{cases}X_{j}=\frac{1}{i} \frac{\partial}{\partial x_{j}}-x_{k+j}\left|D_{x_{n}}\right|, & 1 \leq j \leq k, \\ X_{k+j}=\frac{\partial}{\partial x_{k+j}}, & 1 \leq j \leq k, \\ Y_{s}=\frac{\partial}{\partial x_{2 k+s}}, & 1 \leq s \leq \ell\end{cases}
$$


$X=\left(X_{1}, \ldots, X_{k}, X_{k+1}, \ldots, X_{2 k}\right), Y=\left(Y_{1}, \ldots, Y_{\ell}\right), \mathcal{A}$ is a self adjoint positive definite matrix of size $2 k+\ell$ of pseudo-differential operators of order 0 , and $L$ is a (complex) $2 k+\ell$ dimensional vector of pseudo-differential operators of order 0 .

If $\left(x_{0}, \xi_{0}\right) \in \Sigma$, we denote by $P_{\left(x_{0}, \xi_{0}\right)}\left(x, D_{x}\right)$ the pseudo-differential operator obtained by freezing the coefficients of $(2.4)$ at $\left(x_{0}, \xi_{0}\right)$ :

$$
\begin{gathered}
P_{\left(x_{0}, \xi_{0}\right)}\left(x, D_{x}\right)=\left\langle\mathcal{A}\left(x_{0}, \xi_{0}\right)\left[\begin{array}{c}
X\left(x, D_{x}\right) \\
Y\left(x, D_{x}\right)
\end{array}\right],\left[\begin{array}{c}
X^{*}\left(x, D_{x}\right) \\
Y\left(x, D_{x}\right)
\end{array}\right]\right\rangle \\
\quad+\left\langle L\left(x_{0}, \xi_{0}\right),\left[\begin{array}{c}
X\left(x, D_{x}\right) \\
Y\left(x, D_{x}\right)
\end{array}\right]\right\rangle+\tilde{p}_{1}\left(x_{0}, \xi_{0}\right) T+\tilde{p}_{0}\left(x_{0}, \xi_{0}\right),
\end{gathered}
$$

where $T=\frac{\partial}{\partial x_{n}}$.

Due to $\left(\mathrm{H}_{2}\right)$ it is easy to get an a priori estimate for $P_{\left(x_{0}, \xi_{0}\right)}\left(x, D_{x}\right)$ (see e.g. [7], Chapter XXII):

$$
\begin{aligned}
& \sum_{|\alpha| \leq 2}\left\|X^{\alpha} u\right\|^{2}+\sum_{\beta \leq 2}\left\|Y^{\beta} u\right\|^{2}+\|u\|_{1}^{2} \\
& \quad \leq C\left(\left\|P_{\left(x_{0}, \xi_{0}\right)}\left(x, D_{x}\right) u\right\|^{2}+\|u\|^{2}\right), \quad u \in C_{0}^{\infty},
\end{aligned}
$$

where $C>0$ is independent of $u, \alpha \in \mathbb{Z}_{+}^{2 k}, \beta \in \mathbb{Z}_{+}^{\ell}$ are multi-indices, and $\|\cdot\|_{s}$ means the microlocal $H^{s}$ norm near $\left(x_{0}, \xi_{0}\right)$.

By Assumption $\left(\mathrm{H}_{1}\right),(\mathrm{b})-(\mathrm{d})$, we know that $\Sigma$ is canonically foliated with leaves of dimension $\ell$. If $\left(x_{0}, \xi_{0}\right) \in \Sigma$ let us denote by $\Gamma_{\left(x_{0}, \xi_{0}\right)}$ the leaf through $\left(x_{0}, \xi_{0}\right)$ and by $T_{\left(x_{0}, \xi_{0}\right)} \Gamma_{\left(x_{0}, \xi_{0}\right)}$ its tangent space at $\left(x_{0}, \xi_{0}\right)$. Since we work in a neighborhood of $\left(x_{0}, \xi_{0}\right)$ in $T^{*} \mathbb{R}^{n} \backslash\{0\}$, in the sequel we will identify $\Gamma$ and its tangent space.

We are ready to state our results:

Theorem 2.1. Let $P$ be as above, verifying $\left(\mathrm{H}_{1}\right)$ and $\left(\mathrm{H}_{2}\right)$. Let $\left(x_{0}, \xi_{0}\right) \in \Sigma$ and $W$ be a neighborhood of $\left(x_{0}, \xi_{0}\right)$. Suppose $1 \leq s<2$ and that $\left(x_{0}, \xi_{0}\right) \notin W F_{s}(P u)$; then if $\Gamma_{\left(x_{0}, \xi_{0}\right)} \cap\left(W \backslash\left\{\left(x_{0}, \xi_{0}\right)\right\}\right) \cap W F_{s}(u)=\varnothing$ we have $\left(x_{0}, \xi_{0}\right) \notin W F_{s}(u)$.

Theorem 2.2. Under the same assumptions as in Theorem 2.1, let $s \geq 2$ and $\left(x_{0}, \xi_{0}\right) \notin W F_{s}(P u)$. Then $\left(x_{0}, \xi_{0}\right) \notin W F_{s}(u)$, i.e. $P$ is $G^{s}$-microhypoelliptic.

Remark 2.1. When $s=+\infty$ Theorem 2.2 is the well known result of Hörmander [6], Boutet-Grigis-Helffer [3]. When $s=1$ Theorem 2.1 is due to Métivier [10].

Example. We give here the example of an operator satisfying our assumptions. Assume that the variable $x \in \mathbb{R}^{n}$ is partitioned in three groups which we write $x=\left(x^{\prime}, x^{\prime \prime}, x^{\prime \prime \prime}\right) \in \mathbb{R}^{k} \times \mathbb{R}^{l} \times \mathbb{R}^{n-k-l}$. Consider the operator:

$$
P(x, \xi)=\sum_{j=1}^{k} \mu_{j}\left(D_{x_{j}}^{2}+x_{j}^{2}\left|D^{\prime \prime \prime}\right|^{2}\right)+\sum_{i=k+1}^{k+l} a_{i} D_{x_{i}}^{2}+\alpha\left|D^{\prime \prime \prime}\right|,
$$

where the $\mu_{j}$ 's and the $a_{i}$ 's are positive numbers, $j=1, \ldots, k, i=k+1, \ldots, k+l$, and $\alpha$ is a real (complex) number such that $\alpha+\sum_{j=1}^{k} \mu_{j}\left(2 n_{j}+1\right) \neq 0, n=$ $\left(n_{1}, \ldots, n_{k}\right) \in \mathbb{Z}_{+}^{k}$. The characteristic double manifold for $P$ is given by $\Sigma=$ $\left\{(x, \xi) \in T^{*} \mathbb{R}^{n} \backslash\{0\}\left|x^{\prime}=\xi^{\prime}=0, \xi^{\prime \prime}=0,\right| \xi^{\prime \prime \prime} \mid \neq 0\right\}$. Let $\left(x_{0}, \xi_{0}\right) \in \Sigma$, i.e. $\left(x_{0}, \xi_{0}\right)=\left(0, \bar{x}^{\prime \prime}, \bar{x}^{\prime \prime \prime}, 0,0, \bar{\xi}^{\prime \prime \prime}\right)$, for certain vectors $\bar{x}^{\prime \prime}, \bar{x}^{\prime \prime \prime}$ and $\bar{\xi}^{\prime \prime \prime}$ with $\left|\bar{\xi}^{\prime \prime \prime}\right| \neq 0$; we obtain then that the leaf through $\left(x_{0}, \xi_{0}\right), \Gamma_{\left(x_{0}, \xi_{0}\right)}$, is given by $\Gamma_{\left(x_{0}, \xi_{0}\right)}=\{(x, \xi) \in$ $\left.T^{*} \mathbb{R}^{n} \backslash\{0\} \mid(x, \xi)=\left(0, x^{\prime \prime}, \bar{x}^{\prime \prime \prime}, 0,0, \bar{\xi}^{\prime \prime \prime}\right), x^{\prime \prime} \in \mathbb{R}^{l}\right\}$. It is known that with the preceding assumptions $P$ is $C^{\infty}$ hypoelliptic. On the other hand Theorem 2.1 says that 
$P$ is $G^{s}$ hypoelliptic for $s \geq 2$, whereas Theorem 2.2 states that there is "propagation" of the Gevrey $s$ singularities along the leaf $\Gamma_{\left(x_{0}, \xi_{0}\right)}$ (i.e. in the $x^{\prime \prime}$ variables) if $1 \leq s<2$.

\section{Technical Considerations}

Definition 3.0.1. We say that $\left(x_{0}, \xi_{0}\right) \notin W F_{s}(u), u \in \mathcal{D}^{\prime}, s \geq 1$, if there exists an open conic neighborhood of $\left(x_{0}, \xi_{0}\right), V_{0} \times \Gamma_{0}$, and a constant $C>0$ and a bounded sequence $v_{N} \in \mathcal{E}^{\prime}$, such that for every $N \in \mathbb{N}, v_{N}=u$ in $V_{0}$, and moreover

$$
\left|\hat{v}_{N}(\xi)\right| \leq C^{N}\left(1+\frac{|\xi|^{1 / s}}{N}\right)^{-N}, \quad \xi \in \Gamma_{0} .
$$

In order to prove Theorems 2.1 and 2.2 we shall use the estimate (2.6) and some microlocalizations of high derivatives with respect to characteristic directions.

3.1. The localizing functions. The definition of $W F_{s}$ given in (3.0.1) refers to a fixed conic neighborhood $V_{0} \times \Gamma_{0}$ of $\left(x_{0}, \xi_{0}\right)$, valid for all $N$, and we shall prove (3.0.1) for $u$ and $V_{0} \times \Gamma_{0}$, given (3.0.1) for $P u$ and $\tilde{V}_{0} \times \tilde{\Gamma}_{0}$, with $V_{0} \subset \tilde{V}$ and $\Gamma_{0} \subset \overline{\tilde{\Gamma}}$. In doing this we shall need to nest many such neighborhoods, using carefully chosen cut-off functions, $\varphi(x), \psi(x)$. In fact, once the definition of $W F_{s}$ has been rewritten in terms of $L^{2}$ norms, (2.7) will allow us to estimate derivatives of order $\leq N$ in $V_{0} \times \Gamma_{0}$ in terms of those of order $\leq N / 2$ without changing cut-off functions; at this point we switch to a new pair for the reduction to order $N / 4$ etc., requiring $\log _{2} N$ pairs in all. Actually it is helpful to have two pairs for each step, with two additional pairs initially.

Thus we shall use $2 \log _{2} N+2$ pairs of functions $\{\varphi, \psi\}:\left\{\varphi_{j}, \psi_{j}\right\},\left\{\varphi_{j}^{\prime}, \psi_{j}^{\prime}\right\}$, $j=-1,0,1, \ldots, \log _{2} N$, satisfying the following properties:

$$
\varphi_{j} \quad\left(\psi_{j}\right) \equiv 1 \quad \text { near supp } \varphi_{j} \quad\left(\operatorname{supp} \psi_{j}\right), \quad j=0,1, \ldots, \log _{2} N .
$$

$\varphi_{-1} \equiv 1 \quad$ near $\quad V_{0}, \quad \varphi_{j}\left(\varphi_{j}^{\prime}\right) \in C_{0}^{\infty}(\tilde{V}), \forall j$

$\psi_{-1} \equiv 1$ near $\quad \Gamma_{0} \cap\{|\xi| \geq 2 N\} \quad$ and

$$
\psi_{-1} \equiv 0 \quad \text { for } \quad|\xi| \leq N \text {. }
$$

$$
\begin{array}{ccl}
\psi_{j}(\xi)\left(\psi_{j}^{\prime}(\xi)\right) \equiv 0 \quad \text { for } \quad & |\xi| \leq N / 2^{j} \\
\psi_{j} \in C_{0}^{\infty}(\tilde{\Gamma}) & \text { for every } & j \geq 1
\end{array}
$$

$$
\left|D^{\alpha} \varphi_{j}^{(\prime)}(x)\right| \leq\left(K_{j} N / 2^{j}\right)^{|\alpha|}, \quad|\alpha| \leq 3 N / 2^{j}, \quad j=-1,0, \ldots, \log _{2} N .
$$

$$
\left|D^{\alpha} \psi_{j}^{(\prime)}(\xi)\right| \leq\left(K_{j} N /\left(2^{j}|\xi|\right)\right)^{|\alpha|}, \quad|\alpha| \leq 3 N / 2^{j}, \quad j=-1,0, \ldots, \log _{2} N .
$$

$$
\prod_{j=-1}^{\log _{2} N} K_{j}^{N / 2^{j}} \leq C^{N} .
$$




$$
\text { All the constants } K_{j}, C \text { are independent of } N \text { and depend only on } V_{0} \text {. }
$$

We shall also need a form for the $\varphi_{j}^{(')}$ more adapted to the geometry involved in our problem. If $x \in \mathbb{R}^{n}$, let $x=\left(x^{\prime}, x^{\prime \prime}, x^{\prime \prime \prime}, x_{n}\right)$ denote a partition of the variables according to (2.5), i.e. $x^{\prime} \in \mathbb{R}^{2 k}, x^{\prime \prime} \in \mathbb{R}^{\ell}, x^{\prime \prime \prime} \in \mathbb{R}^{n-2 k-\ell-1}$. Then for every $j$

$$
\varphi_{j}^{(\prime)}(x)=\varphi_{j}^{(\prime) \#}\left(x^{\prime \prime}\right) \tilde{\varphi}_{j}^{(\prime)}\left(x^{\prime}, x^{\prime \prime \prime}, x_{n}\right) .
$$

The construction of the $\varphi_{j}^{(\prime)}(x)$ and $\psi_{j}^{(\prime)}(\xi)$ is easy; moreover these pairs of functions have been used by the second author before ([14]), although the idea of using cutoff functions which behave in an analytic fashion up to a given order is due to L. Ehrenpreis and has been exploited by L. Hörmander, K.G. Andersson, ([5], [1]) and others. We give here a sketch of the construction. Let $\Psi_{i}(t) \equiv 0$ for $|t| \leq N_{i}$, $\Psi_{i}(t) \equiv 1$ for $|t| \geq 2 N_{i}$ and satisfy

$$
\left|D_{t}^{\alpha} \Psi_{i}(t)\right| \leq C^{|\alpha|+1}, \quad \text { for } \alpha \leq 3 N_{i},
$$

where $N_{i}=N / 2^{i}, \Psi_{i} \in C^{\infty}\left(\mathbb{R}^{n}\right)$ and the constant $C$ is independent of $N$ and $i$. Such a function is obtained by convolving the characteristic function of $\left\{|t| \leq 3 N_{i} / 2\right\}$ with $3 N_{i}$ identical non-negative functions of integral one and support in $\{|t| \leq 1 / 6\}$.

Let $U_{0} \subset \subset V_{-1} \subset \subset V_{-1}^{\prime} \subset \subset V_{0} \subset \subset \ldots \subset \subset V_{\log _{2} N}^{\prime} \subset \subset \tilde{V}$, with

$$
\max \left\{\operatorname{dist}\left(V_{i}, V_{i}^{\text {comp }}\right), \operatorname{dist}\left(V_{i}^{\prime}, V_{i+1}^{\text {comp }}\right)\right\}=d_{i},
$$

such that

$$
d_{i}=d_{0} / 2^{i}
$$

Without loss of generality we may take each of the above mentioned nested open sets in product form: $V_{j}^{(\prime) \#} \times \tilde{V}_{j}^{(\prime)}$, where $V_{j}^{(\prime) \#} \subset \mathbb{R}^{\ell}, \tilde{V}_{j}^{(\prime)} \subset \mathbb{R}^{n-\ell}$. Then we construct $\varphi_{i}^{(\prime) \#}\left(x^{\prime \prime}\right), \tilde{\varphi}_{i}^{(\prime)}\left(x^{\prime}, x^{\prime \prime \prime}, x_{n}\right)$ (and hence $\left.\varphi_{i}^{(\prime)}(x)\right)$ just as we $\operatorname{did} \Psi_{i}(t)$, but scaling by a factor $d_{i} N_{i}$ (any positive order derivative of $\Psi_{i}$ had support on a set of size $N_{i}$; now this distance is $\left.d_{0} / 2^{i}\right)$.

The $\psi_{i}^{(\prime)}(\xi)$ are similarly defined by nesting open cones $\Gamma_{0} \subset \subset \tilde{\Gamma}_{-1} \subset \subset \tilde{\Gamma}_{-1}^{\prime} \subset$ $\subset \ldots \subset \subset \tilde{\Gamma}_{\log _{2} N}^{\prime} \subset \subset \tilde{\Gamma}$, with separations on the unit sphere of $e_{i}=e_{0} / 2^{i}$. Thus repeating on the unit sphere the construction of the $\varphi_{i}^{(\prime)}$ - but disregarding the product form - we construct the $\tilde{\psi}_{i}^{(\prime)}$. We then extend the $\tilde{\psi}_{i}^{(\prime)}$ to be homogeneous of degree zero and take the product with $\Psi_{i}(|\xi|)$.

Note that the functions thus obtained, but not the constants, depend on $N$.

3.2. Constants. A note on the use of constants. Any use of the letter $C$ denotes a constant different from line to line, depending only on the dimension of the space and the operator $P$, but independent of $N$ and $u$.

The constants $K_{i}$ or $\tilde{K}_{i}$ will be reserved for constants satisfying (3.1.7) and, like $C$, may change from line to line.

3.3. Underlining. Often we will be interested in the number of terms of a given form which appear in an expansion. This is denoted by underlining a coefficient, 
and may denote an upper bound rather than the exact count:

$$
\begin{aligned}
\left(\frac{\partial}{\partial x}\right)^{a}\left(x^{b} f(x)\right) & =\sum_{c \leq \max \{a, b\}}\left(\begin{array}{l}
a \\
c
\end{array}\right)\left(\begin{array}{l}
b \\
c
\end{array}\right) c ! x^{b-c} f^{(a-c)}(x) \\
& =\sum_{c \leq \max \{a, b\}}{\underline{2^{a} b^{c}}}^{b-c} f^{(a-c)}(x) .
\end{aligned}
$$

Another example, proved in the Appendix and often used below is

$$
x^{\alpha_{1}}\left(\frac{\partial}{\partial x}\right)^{\beta_{1}} \ldots x^{\alpha_{r}}\left(\frac{\partial}{\partial x}\right)^{\beta_{r}}=\sum_{\delta \leq \alpha, \beta} C^{|\beta|}|\alpha|^{|\delta|} x^{\alpha-\delta}\left(\frac{\partial}{\partial x}\right)^{\beta-\delta},
$$

where $\alpha_{i}, \beta_{i}$ are multi-indices, $|\alpha|=\alpha=\sum \alpha_{i},|\beta|=\beta=\sum \beta_{i}$.

3.4. Pseudo-differential operators. We shall use the Gevrey $s$ pseudo-differential operators ( $G^{s}$ pdo's) of Boutet de Monvel and Krée ([4]); i.e. if $v \in C_{0}^{\infty}\left(\mathbb{R}^{n}\right)$,

$$
p\left(x, D_{x}\right) v(x)=\int e^{i x \cdot \xi} p(x, \xi) \hat{v}(\xi) d \xi,
$$

where $d \xi=(2 \pi)^{-n} d \xi, p(x, \xi) \sim \sum_{k} p_{k}(x, \xi)$ and $p(x, \xi)\left(p_{k}(x, \xi)\right)$ is real analytic in $\Omega \times\left(\mathbb{R}^{n} \backslash\{0\}\right), \Omega \subset \mathbb{R}^{n}$ open, and the $p_{k}(x, \xi)$ are positively homogeneous of degree $r-k$ with respect to $\xi$ and satisfy the following: $\forall K \subset \subset \Omega, K$ compact, there exist constants $C, A$ such that for any integer $k$, any $\alpha, \beta \in \mathbb{Z}_{+}^{n}$ and any $x \in K$ we have

$$
\left|D_{x}^{\alpha} D_{\xi}^{\beta} p_{k}(x, \xi)\right| \leq C A^{k+|\alpha+\beta|}|\xi|^{r-k-|\beta|}(k+|\alpha|) !^{s}|\beta| !
$$

and in addition, for any integer $N$ we have

$$
\left|D_{x}^{\alpha} D_{\xi}^{\beta}\left(p(x, \xi)-\sum_{k=0}^{N-1} p_{k}(x, \xi)\right)\right| \leq C A^{N+|\alpha+\beta|}|\xi|^{r-N-|\beta|}(N+|\alpha|) !^{s}|\beta| !
$$

(with $|\xi|^{r-N-|\beta|}$ replaced by $(1+|\xi|)^{r-N-|\beta|}$ if $r-N-|\beta|>0$ ). Here $D_{x}=\frac{1}{i} \partial / \partial x$ and $D_{\xi}=\frac{1}{i} \partial / \partial \xi$.

\section{Gevrey Hypoellipticity}

\subsection{Preliminary remarks.}

Proposition 4.1.1. To prove (3.0.1) for $u$ it suffices to show that for $p \leq \frac{n}{s}+$ $\frac{n+1}{2}$

$$
\left\|T^{p} \psi_{-1}(D) \varphi_{-1}(x) u\right\| \leq C C^{N} N^{p s} .
$$

In other words it suffices to show that for $p \leq N,|\lambda| \leq N$,

$$
\left\|T^{p} \psi_{-1}^{(\lambda)}(D) \varphi_{-1(\lambda)}(x) u\right\| \leq C C^{N} N^{s(p+|\lambda|)}
$$

and that

$$
\begin{aligned}
R_{1, N}(u) & \equiv\left\|T^{p}\left(\psi_{-1}(D) \varphi_{-1}(x)-\sum_{|\lambda| \leq N} \frac{1}{\lambda !}\left(D_{x}^{\lambda} \varphi_{-1}\right)(x)\left(\partial_{\xi}^{\lambda} \psi_{-1}\right)(D)\right) u\right\| \\
& \leq\left(C K_{-1}\right)^{N} N !^{s}
\end{aligned}
$$

where $K_{-1}$ satisfies (3.1.8). 
Proof. First we show that (3.0.1) is implied by the first estimate

$$
\left\|T^{p} \psi_{-1}(D) \varphi_{-1}(x) u\right\| \leq C C^{N} N^{p s}, \quad p \leq \frac{N}{s}+\frac{n+1}{2} .
$$

In fact, replacing $u_{N}$ by $\varphi_{-1}(x) u$-we may assume that $\left(x_{0}, \xi_{0}\right)=\left(0, e_{n}\right)$ and that $u_{N}=u$ in the open neighborhood of $0, U_{0}$, with compact support there-we have the relation that

is equivalent to

$$
\left|\hat{u}_{N}(\xi)\right| \leq C^{N}\left(1+\frac{|\xi|^{1 / s}}{N}\right)^{-N}
$$

$$
\left|\xi_{n}^{k} \hat{u}_{N}(\xi)\right| \leq C^{N} N^{k s}, \quad k \leq \frac{N}{s} .
$$

This is easy to show since, possibly adjusting the constant $C, c|\xi| \leq\left|\xi_{n}\right| \leq c^{\prime}|\xi|$ in a small cone near $\left(0, e_{n}\right)$. The last inequality allows us to show that

$$
\left|\xi_{n}^{k} \psi_{-1}(\xi) \widehat{\varphi_{-1}(x)} u\right| \leq C^{N} N^{k s}, \quad k \leq \frac{N}{s}, \quad \text { if } \quad|\xi| \geq 2 N .
$$

When $|\xi| \leq 2 N$ we have:

$$
\begin{gathered}
\left|\xi_{n}^{k} \int \hat{\varphi}_{-1}(\eta) \hat{u}(\xi-\eta) d \eta\right| \leq C(1+|\xi|)^{k+M} \cdot \sup _{\eta}\left[(1+|\eta|)^{M+n+1}\left|\hat{\varphi}_{-1}(\eta)\right|\right] \\
\leq C^{N} N^{(k+M) s} C^{M+n+1} N^{M+n+1} \leq C^{N} N^{k s}
\end{gathered}
$$

where, by the Paley-Wiener theorem, $|\hat{u}(\eta)| \leq C(1+|\eta|)^{M}, M>0$ depending on $n$. The insertion of a converging factor $(1+|\xi|)^{-n-1}$ together with the above remark that $|\xi| \sim\left|\xi_{n}\right|$ yields the first assertion of Proposition 4.1.1.

Let us now turn to the second part of Proposition 4.1.1. It suffices to prove (4.1.2), since (4.1.1) will follow by general arguments of the calculus of pdo's. We have

$$
\begin{aligned}
& \psi_{-1}\left(D_{x}\right) \varphi_{-1}(x) \sim O p\left(\sum_{|\alpha| \geq 0} \frac{1}{\alpha !} \partial_{\xi}^{\alpha} \psi_{-1} D_{x}^{\alpha} \varphi_{-1}(x)\right) \\
& \sim \sum_{|\alpha| \leq N} \frac{1}{\alpha !} \varphi_{-1}^{(\alpha)}(x) \psi_{-1(\alpha)}\left(D_{x}\right)+\psi_{-1}\left(D_{x}\right) \varphi_{-1}(x)-\left\{\psi_{-1} \circ \varphi_{-1}\right\}_{N}\left(x, D_{x}\right),
\end{aligned}
$$

where we used the notation

$$
\left\{\psi_{-1} \circ \varphi_{-1}\right\}_{N}(x, \xi)=\sum_{0 \leq|\alpha| \leq N} \frac{1}{\alpha !} D_{x}^{\alpha} \varphi_{-1}(x) \partial_{\xi}^{\alpha} \psi_{-1} .
$$

Thus

$$
\begin{aligned}
& \left\|T^{p} \psi_{-1}\left(D_{x}\right) \varphi_{-1}(x) u\right\|_{L^{2}} \\
& \quad \leq \sum_{0 \leq|\lambda| \leq N} \frac{1}{\lambda !}\left\|T^{p} \varphi_{-1(\lambda)}(x) \psi_{-1}^{(\lambda)}\left(D_{x}\right) u\right\|_{L^{2}}+R_{1, N}(u),
\end{aligned}
$$

where $R_{1, N}(u)$ is given by (4.1.2). It is then enough to show that $N^{|\lambda|} \leq C^{N} \lambda$ ! for some positive constant $C$, and for $|\lambda| \leq N$.

This completes the proof of Proposition 4.1.1.

Proposition 4.1.2. Using the same notation as in Proposition 4.1.1, we have that the estimate (4.1.2) holds. 
Proof. Recalling Lemma A.1 of the Appendix we have that

$$
\begin{aligned}
& \left\|R_{1, N}(u)\right\|_{L^{2}}=\left\|T^{p}\left(\psi_{-1}\left(D_{x}\right) \varphi_{-1}(x)-\sum_{0 \leq|\beta| \leq N} \frac{1}{\beta !} \varphi_{-1(\beta)}(x) \psi_{-1}^{(\beta)}\left(D_{x}\right)\right) u\right\|_{L^{2}} \\
& =\sum_{c_{1}} \underline{C^{p+n}}\left\|\int e^{i x \cdot \xi}\left(\int r_{c_{1}}(x, \eta, \xi) d \eta\right) \hat{u}(\xi) d \xi\right\|
\end{aligned}
$$

where $c_{1}$ takes the values specified below and

$$
\begin{aligned}
& r_{c_{1}}(x, \eta, \xi)=\sum_{|\varepsilon|=N} \frac{(1+|\eta|)^{-n-1}}{\varepsilon !} e^{i x \cdot \xi}\left(\varphi_{-1\left(\varepsilon+c_{1}+b_{2}\right)}\right)^{\wedge}(\eta) \\
& \times\left(\int_{0}^{1} \psi_{-1}^{(\varepsilon)}(\xi+\rho \eta)(\xi+\rho \eta)^{p e_{n}-c_{1}+b_{1}}(1-\rho)^{\left|c_{1}+\varepsilon\right|} d \rho\right)
\end{aligned}
$$

here $\left|b_{1}+b_{2}\right|=n+1, c_{1} \leq p e_{n}+b_{1}$, and we have to study the symbol $\int r_{c_{1}}(x, \eta, \xi) d \eta$.

Now $D_{x}^{\mu} r_{c_{1}}$ has a similar expression where

$$
\left(\varphi_{-1\left(\varepsilon+c_{1}+b_{2}\right)}\right)^{\wedge} \quad \text { is replaced by } \quad \eta^{\mu}\left(\varphi_{-1\left(\varepsilon+c_{1}+b_{2}\right)}\right)^{\wedge},
$$

which is rapidly decreasing in $\eta$ by the Paley-Wiener theorem. Furthermore the part under the integral sign is bounded by

$$
\sup _{\sigma \in \mathbb{R}^{n},|\varepsilon|=N}\left|\sigma^{\varrho e_{n}-c_{1}+b_{1}} \psi_{-1}^{(\varepsilon)}(\sigma)\right| \leq C^{N} N !
$$

by (3.1.3) and (3.1.6). Hence we consider

$$
\begin{gathered}
\sum_{|\mu| \leq n+1} \int\left|D_{x}^{\mu} r_{c_{1}}(x, \eta, \xi)\right| d x d \eta \\
=\sum_{|a| \leq n+1} \int \frac{1}{\left(1+|x|^{2}\right)^{(n+1) / 2}}\left(1+|x|^{2}\right)^{(n+1) / 2}\left|D_{x}^{\mu} r_{c_{1}}(x, \eta, \xi)\right| d \eta d x .
\end{gathered}
$$

It is easily seen that integrating by part with respect to $\eta$ we get an absolutely convergent integral. We may then conclude that the symbol $\int r_{C_{1}} d \eta$ is $L_{2}$ continuous; thus

$$
\left\|R_{1, N}(u)\right\|_{L^{2}} \leq C^{N}\|u\|_{L^{2}} N \text { ! }
$$

where $C=C^{\prime} K_{1}, K_{1}$ given by (3.1.5) - (3.1.8).

To deduce the microlocal Gevrey regularity of $u$ we shall obtain an upper bound for a slightly more complicated expression. First some notation. Recalling the definition (2.5) let us denote by $X=\left(X^{\prime}, X^{\prime \prime}\right)$ the symplectic vector fields: $X_{j}^{\prime}=$ $X_{j}=\frac{\partial}{\partial x_{j}}-x_{j+k} \frac{\partial}{\partial x_{n}}, X_{j}^{\prime \prime}=X_{j+k}=\frac{\partial}{\partial x_{j+k}}, j=1, \cdots, k, Y_{s}=\frac{\partial}{\partial x_{2 k+s}}, 1 \leq s \leq \ell$, $T=\frac{\partial}{\partial x_{n}}$. We shall also denote by $Z$ either an $X$ - or a $Y$-vector field.

If $I \in \mathbb{Z}_{+}^{2 k+\ell}$ is a multi-index, the expression $Z^{I}$ means $X_{1}^{I_{1}} X_{2}^{I_{2}} \ldots X_{2 k}^{I_{2 k}} Y_{2 k+1}^{I_{2 k+1}}$ $\ldots Y_{2 k+\ell}^{I_{2 k+\ell}}$, and similarly $X^{I}, I \in \mathbb{Z}_{+}^{2 k}$, means $X_{1}^{I_{1}} \ldots X_{2 k}^{I_{2 k}}$. Analogously we write $X^{\prime \alpha^{\prime}}, X^{\prime \prime \alpha^{\prime \prime}} \ldots$ etc, for $\alpha^{\prime}, \alpha^{\prime \prime} \in \mathbb{Z}_{+}^{k}$. 

by

In the proof of the microlocal regularity the quantity in (4.1.1) will be replaced

$$
\sup _{|J| \leq 2}\left\|Z^{J} T^{p} \varphi_{-1(\lambda)}(x) \psi_{-1}^{(\lambda)}\left(D_{x}\right) u\right\|_{L_{2}} \leq C^{N+1} N^{s(p+|\lambda|)}, \quad|\lambda| \leq N, p \leq N .
$$

To proceed further a more effective microlocalization will be needed.

\subsection{Effective localization of powers of $T$.}

Definition 4.2.1. For any $\varphi(x), \psi\left(D_{x}\right), p \geq 0$, set

$$
\left(T^{p}\right)_{\varphi \psi}=\sum_{|\alpha+\beta| \leq p} \frac{(-1)^{|\alpha|}}{\alpha ! \beta !} X^{\prime \prime \alpha} X^{\prime \beta} T^{p-|\alpha+\beta|} \circ a d_{X^{\prime}}^{\alpha} a d_{X^{\prime \prime}}^{\beta}(\varphi \psi),
$$

where $X^{\prime \prime \alpha}$ has been defined above,

$$
a d_{X_{i}}^{\alpha_{i}}(W)=\underbrace{\left[X_{i},\left[X_{i}, \ldots,\left[X_{i}, W\right] \ldots\right]\right]}_{\alpha_{i} \text { times }}
$$

and $a d_{X^{\prime}}^{\alpha}(W)=a d_{X_{1}}^{\alpha_{1}} \circ \cdots \circ a d_{X_{k}}^{\alpha_{k}}(W)$.

Remark 4.2.1. Recalling the definition (3.1.9) of $\varphi_{j}$, we have

$$
\left(T^{p}\right)_{\varphi_{j} \psi_{j}}=\varphi_{j}^{\#}\left(x^{\prime \prime}\right)\left(T^{p}\right)_{\tilde{\varphi}_{j} \psi_{j}} .
$$

Definition 4.2.2. We shall write $Z^{p}$ for $Z^{I}$ with $|I|=p$ and $X^{p}$ for $X^{J},|J|=p$ and so on. Analogously $a d_{X}^{p}$ means $a d_{X}^{\alpha},|\alpha|=p$ etc. If $F$ is an operator, we shall write

$$
F \equiv{ }_{p} 0
$$

if

$$
F=\underline{C^{p}} \frac{1}{p !} X^{p} a d_{X}^{p}(\varphi \psi)
$$

where $C$ is a universal constant. The index $p$ will be dropped if there is no possibility of misunderstanding.

Proposition 4.2.1. We have the following properties of the microlocalizations defined in (4.2.1):

$$
\begin{gathered}
{\left[X_{j}^{\prime},\left(T^{p}\right)_{\varphi \psi}\right] \equiv_{p} 0, \quad 1 \leq j \leq k} \\
{\left[X_{j}^{\prime \prime},\left(T^{p}\right)_{\varphi \psi}\right] \equiv_{p} X_{j}^{\prime \prime}\left(T^{p-1}\right)_{a d_{T}(\varphi \psi)}, \quad 1 \leq j \leq k ;} \\
{\left[Y_{j},\left(T^{p}\right)_{\varphi \psi}\right]=\left(T^{p}\right)_{a d_{Y}(\varphi) \psi}, \quad 1 \leq j \leq \ell .}
\end{gathered}
$$

Proof. This proof is accomplished by a calculation. (4.2.7) is almost obvious since the vector field $Y_{j}$ commutes with all the $X^{\prime}, X^{\prime \prime}$. Thus we are left with the verification of (4.2.5) and (4.2.6). As for (4.2.5) we have

$$
\left[X_{j}^{\prime},\left(T^{p}\right)_{\varphi \psi}\right]=\left[X_{j}^{\prime}, \sum_{|\alpha+\beta| \leq p} A_{\alpha \beta}^{(p)} B_{\alpha \beta}(\varphi \psi)\right]
$$

where

$$
A_{\alpha \beta}^{(p)}=\frac{(-1)^{|\alpha|}}{\alpha ! \beta !} X^{\prime \prime \alpha} X^{\prime \beta} T^{p-|\alpha+\beta|}
$$




$$
B_{\alpha \beta}(\varphi \psi)=a d_{X^{\prime}}^{\alpha} a d_{X^{\prime \prime}}^{\beta}(\varphi \psi)
$$

Now

so that

$$
\begin{aligned}
& {\left[X_{j}^{\prime}, A_{\alpha \beta}^{(p)}\right]=}-\frac{(-1)^{\left|\alpha-e_{j}\right|}}{\left(\alpha-e_{j}\right) ! \beta !} X^{\prime \prime \alpha-e_{j}} X^{\prime \beta} T^{p-\left|\alpha-e_{j}+\beta\right|} \\
&=-A_{\alpha-e_{j} \beta}^{(p)}, \\
& {\left[X_{j}^{\prime}, B_{\alpha \beta}(\varphi \psi)\right]=a d_{X^{\prime}}^{\alpha+e_{j}} a d_{X^{\prime \prime}}^{\beta}(\varphi \psi)=B_{\alpha+e_{j} \beta}(\varphi \psi), }
\end{aligned}
$$

$$
\begin{aligned}
{\left[X_{j}^{\prime},\left(T^{p}\right)_{\varphi \psi}\right]=} & -\sum_{|\alpha+\beta| \leq p-1} A_{\alpha \beta}^{(p)} B_{\alpha+e_{j} \beta}(\varphi \psi) \\
& +\sum_{|\alpha+\beta| \leq p} A_{\alpha \beta}^{(p)} B_{\alpha+e_{j} \beta}(\varphi \psi) \\
= & \sum_{|\alpha+\beta|=p} A_{\alpha \beta}^{(p)} B_{\alpha+e_{j} \beta}(\varphi \psi) \equiv_{p} 0 .
\end{aligned}
$$

Let us now turn to (4.2.6). This time

$$
\begin{aligned}
{\left[X_{j}^{\prime \prime}, A_{\alpha \beta}^{(p)}\right] } & =-\frac{(-1)^{|\alpha|}}{\alpha !\left(\beta-e_{j}\right) !} X^{\prime \prime \alpha} X^{\prime \beta-e_{j}} T^{p-\left|\alpha+\beta-e_{j}\right|} \\
& =-A_{\alpha \beta-e_{j}}^{(p)}
\end{aligned}
$$

and since $a d_{X_{j}^{\prime \prime}} a d_{X^{\prime}}^{\alpha}(v)=a d_{X^{\prime}}^{\alpha} a d_{X_{j}^{\prime \prime}}(v)-\alpha_{j} a d_{X^{\prime}}^{\alpha-e_{j}} a d_{T}(v)$,

$$
\left[X_{j}^{\prime \prime}, B_{\alpha \beta}(\varphi \psi)\right]=a d_{X^{\prime}}^{\alpha} a d_{X^{\prime \prime}}^{\beta+e_{j}}(\varphi \psi)-\alpha_{j} a d_{X^{\prime}}^{\alpha-e_{j}} a d_{X^{\prime \prime}}^{\beta} a d_{T}(\varphi \psi),
$$

so that

$$
\begin{aligned}
& {\left[X_{j}^{\prime \prime},\left(T^{p}\right)_{\varphi \psi}\right]=}-\sum_{|\alpha+\beta| \leq p-1} A_{\alpha \beta}^{(p)} B_{\alpha} \beta+e_{j}(\varphi \psi)+\sum_{|\alpha+\beta| \leq p} A_{\alpha \beta}^{(p)} B_{\alpha \beta+e_{j}}(\varphi \psi) \\
&+\sum_{|\alpha+\beta| \leq p} \frac{(-1)^{\left|\alpha-e_{j}\right|}}{\left(\alpha-e_{j}\right) ! \beta !} X_{j}^{\prime \prime} X^{\prime \prime \alpha-e_{j}} X^{\prime \beta} T^{p-1-\left|\alpha-e_{j}+\beta\right|} \\
& \circ a d_{X^{\prime}}^{\alpha-e_{j}} a d_{X^{\prime \prime}}^{\beta} a d_{T}(\varphi \psi) \\
& \equiv_{p} \quad X_{j}^{\prime \prime}\left(T^{p-1}\right)_{a d_{T}(\varphi \psi)}
\end{aligned}
$$

which proves the assertion.

Proposition 4.2.1 can be iterated giving

Proposition 4.2.2. We have, for $r, p \in \mathbb{Z}_{+}$

$$
\begin{aligned}
{\left[X^{r},\left(T^{p}\right)_{\varphi \psi}\right] } & =\sum_{\ell=1}^{r}(-1)^{\ell-1}\left(\begin{array}{l}
r \\
\ell
\end{array}\right) X^{r}\left(T^{p-\ell}\right)_{a d_{T}^{\ell}(\varphi \psi)} \\
& +\sum_{\ell=0}^{r-1} \sum_{k=0}^{r-\ell} \underline{C}^{p-\ell} \frac{1}{(p-\ell) !} X^{p+r-\ell-k-1} a d_{X, T}^{p+k+1}(\varphi \psi),
\end{aligned}
$$

where $a d_{X, T}^{k}(v)=a d_{X}^{k_{1}} a d_{T}^{k_{2}}(v), k_{1}+k_{2}=k$. When $X^{r}=X^{\prime r}$, the first term on the right hand side of (4.2.8) is missing. 
Proof. This proof is accomplished by induction with a direct calculation. (4.2.8) is obviously true when $r=1$. Suppose (4.2.8) holds for a certain value of $r$ and that every $X=X^{\prime \prime}$. Then

$$
\begin{aligned}
{\left[X^{r+1},\left(T^{p}\right)_{\varphi \psi}\right]=} & X\left[X^{r},\left(T^{p}\right)_{\varphi \psi}\right]+\left[X,\left(T^{p}\right)_{\varphi \psi}\right] X^{r} \\
= & \sum_{\ell=1}^{r}(-1)^{\ell-1}\left(\begin{array}{l}
r \\
\ell
\end{array}\right) X^{r+1}\left(T^{p-\ell}\right)_{a d_{T}^{\ell}(\varphi \psi)}+X^{r+1}\left(T^{p-1}\right)_{a d_{T}(\varphi \psi)} \\
& -\sum_{\ell=1}^{r}(-1)^{\ell-1}\left(\begin{array}{l}
r \\
\ell
\end{array}\right) X^{r+1}\left(T^{p-\ell-1}\right)_{a d_{T}^{\ell+1}(\varphi \psi)} \\
& -\sum_{\ell=0}^{r-1} \sum_{k=0}^{r-\ell} \underline{C}^{p-1-\ell} \frac{1}{(p-1-\ell) !} X^{p+r-\ell-k-1} a d_{X, T}^{p+k+1}(\varphi \psi) \\
& +\sum_{\ell=0}^{r-1} \sum_{k=0}^{r-\ell} \underline{C}^{p-\ell} \frac{1}{(p-\ell) !} X^{p+r-\ell-k} a d_{X, T}^{p+k+1}(\varphi \psi) \\
& +\underline{C}^{p} \frac{1}{p !} X^{p+r} a d_{X}^{p+1}(\varphi \psi)-\underline{C}^{p} \frac{1}{p !} X^{p}\left[X^{r}, a d_{X}^{p+1}(\varphi \psi)\right] .
\end{aligned}
$$

Now taking into account that

$$
\left[X^{r}, a d_{X}^{p+1}(\varphi \psi)\right]=\sum_{k=1}^{r}(-1)^{k-1}\left(\begin{array}{l}
r \\
k
\end{array}\right) X^{r-k} a d_{X}^{p+1+k}(\varphi \psi),
$$

we get the conclusion.

4.3. Using suitably microlocalized norms. The next step will be to replace $T^{p} \varphi_{-1(\lambda)} \psi_{-1}^{(\lambda)}$ in (4.1.1) by the localization $\left(T^{p}\right)_{\varphi_{0} \psi_{0}}$.

Recall that $\varphi_{0} \equiv 1$ on a neighborhood of $\operatorname{supp} \varphi_{-1}$ and that $\psi_{0} \equiv 1$ near $\operatorname{supp} \psi_{-1}$. Then we may write:

$$
\begin{aligned}
Z^{J} & T^{p} \varphi_{-1(\lambda)} \psi_{-1}^{(\lambda)}\left(D_{x}\right) \\
& =\sum_{p^{\prime} \leq p}\left(\begin{array}{c}
p \\
p^{\prime}
\end{array}\right) Z^{J} \varphi_{-1\left(\lambda+\left(p-p^{\prime}\right) e_{n}\right)}(x) \psi_{-1}^{(\lambda)}\left(D_{x}\right) T^{p^{\prime}} \\
& =\sum_{p^{\prime} \leq p} \sum_{J^{\prime} \leq J}\left(\begin{array}{c}
p \\
p^{\prime}
\end{array}\right)\left(\begin{array}{c}
J \\
J^{\prime}
\end{array}\right) \varphi_{-1\left(\lambda+p-p^{\prime}+J-J^{\prime}\right)}(x) Z^{J^{\prime}} \psi_{-1}^{(\lambda)} T^{p^{\prime}},
\end{aligned}
$$

where $\varphi_{-1\left(\lambda+p-p^{\prime}+J-J^{\prime}\right)}(x)=Z^{J-J^{\prime}} T^{p-p^{\prime}} \varphi_{-1(\lambda)}(x)$. From now on we shall often denote as a derivative of $\varphi_{-1}$ such a blend of derivatives of $\varphi_{-1}$.

Using the above notation we have

Proposition 4.3.1. In order to prove the estimate (4.1.6) it suffices to show that

$$
\begin{aligned}
& \sup _{\substack{p^{\prime} \leq p \\
J^{\prime} \leq J}}\left\|\varphi_{-1\left(\lambda+p-p^{\prime}+J-J^{\prime}\right)}(x) \psi_{-1}^{(\lambda)}\left(D_{x}\right) Z^{J^{\prime}}\left(T^{p^{\prime}}\right)_{\varphi_{0}} \psi_{0} u\right\|_{L^{2}} \\
& \quad \leq C^{N+1} N^{s(p+|\lambda|+|J|)}
\end{aligned}
$$

where $|J| \leq 2,|\lambda| \leq N, p \leq N$. 
Proof. From (4.3.1) we obtain

$$
\begin{aligned}
& Z^{J} T^{p} \varphi_{-1(\lambda)}(x) \psi_{-1}^{(\lambda)}\left(D_{x}\right) \\
& =\sum_{p^{\prime} \leq p} \sum_{J^{\prime} \leq J}\left(\begin{array}{c}
p \\
p^{\prime}
\end{array}\right)\left(\begin{array}{c}
J \\
J^{\prime}
\end{array}\right) \varphi_{-1\left(\lambda+p-p^{\prime}+J-J^{\prime}\right)}(x) \\
& \quad \cdot\left(\psi_{-1}^{(\lambda)}\left(D_{x}\right) Z^{J^{\prime}} T^{p^{\prime}}+\left[Z^{J^{\prime}}, \psi_{-1}^{(\lambda)}\left(D_{x}\right)\right] T^{p^{\prime}}\right) \\
& =\sum_{p^{\prime} \leq p} \sum_{J^{\prime} \leq J}\left(\begin{array}{c}
p \\
p^{\prime}
\end{array}\right)\left(\begin{array}{c}
J \\
J^{\prime}
\end{array}\right) \varphi_{-1\left(\lambda+p-p^{\prime}+J-J^{\prime}\right)}(x) \psi_{-1}^{(\lambda)}\left(D_{x}\right) Z^{J^{\prime}}\left(T^{p^{\prime}}\right)_{\varphi_{0} \psi_{0}} \\
& \quad+\sum_{\substack{p^{\prime} \leq p \\
J^{\prime} \leq J}}\left(\begin{array}{c}
p \\
p^{\prime}
\end{array}\right)\left(\begin{array}{c}
J \\
J^{\prime}
\end{array}\right) \varphi_{-1\left(\lambda+p-p^{\prime}+J-J^{\prime}\right)}(x) \\
& \quad \cdot\left(\psi_{-1}^{(\lambda)}\left(D_{x}\right) Z^{J^{\prime}} \circ\left(T^{p^{\prime}}-\left(T^{p^{\prime}}\right)_{\varphi_{0} \psi_{0}}\right)+\left[Z^{J^{\prime}}, \psi_{-1}^{(\lambda)}\left(D_{x}\right)\right] T^{p^{\prime}}\right) \\
& =A_{1}+A_{2}+A_{3}
\end{aligned}
$$

Clearly (4.3.2) means that $A_{1} \leq C^{N+1} N^{s(p+|\lambda|+|J|)}$ which, in turn, implies (4.1.6) provided we show that

$$
A_{i} \leq C^{N+1} N^{s(p+|\lambda|+|J|)}, \quad i=2,3 .
$$

Let us start considering $A_{3}$. Modulo constants bounded by $C^{N}$, the generic term appearing in the sum contained in $A_{3}$ has the form

$$
\varphi_{-1\left(\lambda_{1}\right)}(x)\left[Z^{J^{\prime}}, \psi_{-1}^{(\lambda)}\right] T^{p^{\prime}}
$$

where $\lambda_{1}$ is a suitable multi-index. The term in (4.3.5) can be rewritten as (see equation (A.2) for the definition of $\{\cdot\}_{M}$ )

$$
-\left[\varphi_{-1\left(\lambda_{1}\right)}(x) \psi_{-1}^{(\lambda)}\left(D_{x}\right) \circ Z^{J^{\prime}}-\left\{\varphi_{-1\left(\lambda_{1}\right)}(x) \psi_{-1}^{(\lambda)}\left(D_{x}\right) \circ Z^{J^{\prime}}\right\}_{1}\right] T^{p^{\prime}} .
$$

Applying this operator to a smooth function $u$, by Lemma (A.1) we obtain that the result can be expressed as

$$
\underline{C^{p^{\prime}+n}} \int e^{i x \xi} \int r_{c_{1}}(x, \eta, \xi) d \eta \hat{u}(\xi) d \xi
$$

where

$$
\begin{gathered}
r_{c_{1}}(x, \eta, \xi)=\sum_{|\varepsilon|+1}(1+|\eta|)^{-n-1} Z^{J^{\prime}\left(\widehat{\varepsilon+b_{2}}+c_{1}\right)}(\eta, \xi) e^{i x \eta} \\
\cdot \int_{0}^{1} \varphi_{-1\left(\lambda_{1}\right)}(x) \psi_{-1}^{(\lambda+\varepsilon)}(\xi+\varrho \eta)(\xi+\varrho \eta)^{b_{1}-c_{1}}(1-\varrho)^{\left|c_{1}+\varepsilon\right|} d \varrho .
\end{gathered}
$$

Some explanation is in order here: $\left|b_{1}+b_{2}\right|=p^{\prime}+n+1, c_{1} \leq b_{1}$, and we denoted the Fourier transform by $\left.Z^{J^{\prime}\left(\varepsilon+b_{2}\right.}+c_{1}\right)(\eta, \xi)$ with respect to $X$ of the $\left(\varepsilon+b_{2}+c_{1}\right)$ derivative of the symbol $Z^{J^{\prime}}(x, \xi)$ of the $J^{\prime}$-th power of $X$ or $Y$. It is worth noting that this Fourier transform could give a derivative of the $\delta$-distribution in the $\eta$-variable, since the $X^{\prime}$ 's have polynomial coefficients. On the other hand, going back to (4.1.6), since $Z^{J} T^{p}$ are local operators and $\varphi_{-1}^{\lambda_{1}}(x)$ has compact support contained in $V_{-1}$, we may think of smearing out each $Z^{J}$ by premultiplying it by a smooth function with compact support being identically 1 in a neighborhood of 
$\operatorname{supp} \varphi_{-1}$, e.g. by $\varphi_{-1}^{\prime}$. In such a way we obtain symbols $Z^{J^{\prime}}(x, \xi)$ which are polynomials with respect to $\xi$ and compactly supported in $x$. Thus $Z^{J^{\prime}\left(\varepsilon+b_{2}+c_{1}\right)}(\eta, \xi)$ is a polynomial in $\xi$ of order $\leq\left|J^{\prime}\right|$ with rapidly decreasing coefficients in the $\eta$ variables. Hence $r_{c_{1}}$ is given by a sum of terms of the form:

$$
e^{i x \cdot \eta} \chi(\eta) \xi^{\alpha} \int_{0}^{1} \varphi_{-1\left(\lambda_{1}\right)}(x) \psi_{-1}^{(\lambda+\varepsilon)}(\xi+\varrho \eta)(\xi+\varrho \eta)^{b_{1}-c_{1}}(1-\varrho)^{\left|c_{1}+\varepsilon\right|} d \varrho,
$$

where $|\alpha| \leq\left|J^{\prime}\right|$. Writing $\xi=\xi+\varrho \eta-\varrho \eta$ we may write (4.3.6) as a sum, modulo constants of the strength $C^{N}$, of terms of the form

$$
\begin{aligned}
& e^{i x \cdot \eta} \chi(\eta) \eta^{\beta} \varphi_{-1\left(\lambda_{1}\right)}(x) \\
& \quad \cdot \int_{0}^{1} \psi_{-1}^{(\lambda+\varepsilon)}(\xi+\varrho \eta)(\xi+\varrho \eta)^{b_{1}-c_{1}+\alpha-\beta}(1-\varrho)^{\left|c_{1}+\varepsilon\right|} \varrho^{|\beta|} d \varrho
\end{aligned}
$$

where $|\beta| \leq|\alpha| \leq\left|J^{\prime}\right| \leq|J|$. The term under the integral sign in (4.3.7) can be estimated by $N^{s(p+|\lambda|+|J|)}$ modulo the $N$-th power of a constant and since $\varphi_{-1}$ has compact support the symbol $\int r_{c_{1}}(x, \eta, \xi) d \eta$ is $L^{2}$ continuous, thus yielding the desired conclusion. Next we show the estimate in (4.3.4) when $i=2$. Again applying the generic term of $A_{2}$ in (4.3.3) to a smooth function $u$ we have to estimate the $L^{2}$ norm of a sum of terms of the type

$$
\varphi_{-1\left(\lambda+p-p^{\prime}+J-J^{\prime}\right)}(x) \psi_{-1}^{(\lambda)}\left(D_{x}\right) Z^{J^{\prime}}\left(T^{p^{\prime}}-\left(T^{p^{\prime}}\right)_{\varphi_{0} \psi_{0}}\right)(u) .
$$

Recalling the definition of $\left(T^{p^{\prime}}\right)_{\varphi_{0} \psi_{0}}$, modulo $N$-th power of a suitable constant, the quantity in (4.3.8) is a sum of terms of the form

$$
\frac{1}{r !} \varphi_{-1\left(\lambda+p-p^{\prime}+J-J^{\prime}\right)}(x) \psi_{-1}^{(\lambda)}\left(D_{x}\right) T^{p^{\prime}-r} Z^{r+J^{\prime}} a d_{X}^{r}\left(1-\varphi_{0} \psi_{0}\right) u,
$$

where $r \leq p^{\prime} \leq p \leq N$. Denote by $h_{r}(x, \xi)$ the symbol of the operator $Z^{r+J^{\prime}} a d_{X}^{r}\left(1-\varphi_{0} \psi_{0}\right)$. Then supp $h_{r}(x, \xi) \subset \complement\left(V_{0} \times \Gamma_{0}\right)$, while

$$
\operatorname{supp} \varphi_{-1\left(\lambda+p-p^{\prime}+J-J^{\prime}\right)}(x) \psi_{-1}^{(\lambda)}(\xi) \varsubsetneqq \operatorname{Int}\left[V_{0} \times \Gamma_{0}\right] .
$$

Hence the quantity in (4.3.9) can be rewritten as

$$
\frac{1}{r !} \varphi_{-1\left(\lambda+p-p^{\prime}+J-J^{\prime}\right)}(x)\left[\psi_{-1}^{(\lambda)}\left(D_{x}\right), h_{r}\left(x, D_{x}\right)\right] u,
$$

since

$$
\varphi_{-1\left(\lambda+p-p^{\prime}+J-J^{\prime}\right)}(x) h\left(x, D_{x}\right) \psi_{-1}^{(\lambda)}\left(D_{x}\right)=0 .
$$

Now by the same argument on the support of $h$ and $\varphi_{-1} \psi_{-1}$ we have that

$$
\begin{aligned}
\varphi_{-1\left(\lambda+p-p^{\prime}+J-J^{\prime}\right)} \sigma\left(\left[\psi_{-1}^{(\lambda)}\left(D_{x}\right), h_{r}\left(x, D_{x}\right)\right]\right) \\
=\varphi_{-1\left(\lambda+p-p^{\prime}+J-J^{\prime}\right)} \sigma\left(\psi_{-1}^{(\lambda)}\left(D_{x}\right) h_{r}\left(x, D_{x}\right)\right. \\
\left.\quad-\sum_{|\beta|<N} \frac{1}{\beta !} \psi_{-1}^{(\lambda+\beta)}\left(D_{x}\right) h_{(\beta)}\left(x, D_{x}\right)\right)
\end{aligned}
$$


since

$$
\varphi_{-1\left(\lambda+p-p^{\prime}+J-J^{\prime}\right)} \sum_{1<|\beta|<N} \frac{1}{\beta !} \psi_{-1}^{(\lambda+\beta)}(\xi) h_{(\beta)}(x, \xi)=0 .
$$

The conclusion then follows using Lemma A.1 in the Appendix and arguing as above.

Corollary 4.3.1. In order to prove (4.3.2) it suffices to show that

$$
\sup _{|J| \leq 2}\left\|Z^{J}\left(T^{p}\right)_{\varphi_{0} \psi_{0}} u\right\|_{L^{2}} \leq C^{N+1} N^{s p} .
$$

Proof. From (3.1.5) and (3.1.6) we have that

$$
\left\|\varphi_{-1\left(\lambda_{1}\right)}(x) w\right\|_{L^{2}} \leq C^{\left|\lambda_{1}\right|} K_{-1}^{\left|\lambda_{1}\right|} N^{\left|\lambda_{1}\right|}\|w\|_{L^{2}}
$$

and

$$
\left\|\psi_{-1}^{\left(\lambda_{2}\right)}(x) w\right\|_{L^{2}} \leq C^{\left|\lambda_{2}\right|} K_{-1}^{\left|\lambda_{2}\right|}\|w\|_{L^{2}}
$$

The assertion then follows combining these two estimates.

Actually we shall need to estimate more general derivatives of the microlocalizing functions, due to the interactions of the vector fields $Z$ with the cut-off functions. First some notation:

Definition 4.3.1. Let $Z$ be a vector field on $T^{*} \mathbb{R}^{n}$. We denote by $Z_{\sigma}$ a map from $\mathrm{Op} S^{m}\left(\mathbb{R}^{m}\right) \longrightarrow \mathrm{Op} S^{m}\left(\mathbb{R}^{n}\right)$ defined by

$$
Z_{\sigma} h\left(x, D_{x}\right)=\operatorname{Op}(Z(h(x, \xi)))\left(x, D_{x}\right)
$$

when $h(x, \xi) \in S^{m}\left(\mathbb{R}^{n}\right)$.

Remark 4.3.1. We have

$$
a d_{X_{j}^{\prime}}(h)=\tilde{X}_{j \sigma}^{\prime} h, \quad 1 \leq j \leq k
$$

where

where

$$
\begin{gathered}
\tilde{X}_{j}^{\prime}=\frac{\partial}{\partial x_{j}}-x_{j+k} \frac{\partial}{\partial x_{n}}+\xi_{n} \frac{\partial}{\partial \xi_{j+k}} \\
a d_{X_{j}^{\prime \prime}}(h)=\tilde{X}_{j \sigma}^{\prime \prime} h, \quad 1 \leq j \leq k
\end{gathered}
$$

and

$$
\tilde{X}_{j}^{\prime \prime}=X_{j}^{\prime \prime}=\frac{\partial}{\partial x_{j+k}}
$$

where

$$
a d_{X_{s}}(h)=\tilde{Y}_{s \sigma} h, \quad 1 \leq s \leq k,
$$

$$
\tilde{Y}_{s}=\frac{\partial}{\partial x_{2 k+s}} .
$$

Remark 4.3.2. By the preceding remark we have

$$
a d_{Y}^{\alpha} a d_{X^{\prime}}^{\beta} a d_{X^{\prime \prime}}^{\gamma}(h)=\tilde{Y}_{\sigma}^{\alpha} \tilde{X}_{\sigma}^{\prime} \tilde{X}_{\sigma}^{\prime \prime}(h)
$$

and in particular

$$
B_{\alpha \beta}(\varphi \psi)=a d_{X^{\prime}}^{\alpha} a d_{X^{\prime \prime}}^{\beta}(\varphi \psi)=\tilde{X}_{\sigma}^{\prime \alpha} \tilde{X}_{\sigma}^{\prime \prime \beta}(\varphi \psi) .
$$

Equation (4.3.12) can be rewritten as

$$
a d_{Z}^{\alpha}(h)=\tilde{Z}_{\sigma}^{\alpha} h .
$$


To define our generalized derivatives we shall need the following vector fields.

Definition 4.3.2. Write

$$
\begin{gathered}
W_{j}^{\prime}=\frac{\partial}{\partial x_{j}}, \quad 1 \leq j \leq k, 2 k<j \leq n, \\
W_{j+k}^{\prime}=\frac{\partial}{\partial x_{j+k}}-x_{j} \frac{\partial}{\partial x_{n}}, \quad 1 \leq j \leq k, \\
W_{j}^{\prime \prime}=\xi_{n} \frac{\partial}{\partial \xi_{j+k}}, \quad 1 \leq j \leq k, 2 k<j \leq n, \\
W=\left(W^{\prime}, W^{\prime \prime}\right), \\
\Xi_{j}=\frac{\partial}{\partial \xi_{j}}, \quad 1 \leq j \leq n-1 \\
\Xi_{n}=\frac{\partial}{\partial \xi_{n}}-i \sum_{j=1}^{n} x_{j} \frac{\partial}{\partial \xi_{k+j}} .
\end{gathered}
$$

Note that $\operatorname{span}[W, \Xi]=\operatorname{span}\left[\tilde{Z}, \frac{\partial}{\partial x}\right]$ and $\operatorname{span}[\Xi]=\operatorname{span}\left[\frac{\partial}{\partial \xi}\right]$. Moreover

$$
\left[W_{j}, \tilde{Z}_{k}\right]=0 \text {, }
$$

so that

$$
\begin{aligned}
W_{j, \sigma} B_{\alpha \beta}(\varphi \psi) & =B_{\alpha \beta}\left(W_{j, \sigma}(\varphi \psi)\right), \\
\Xi_{j, \sigma} B_{\alpha \beta}(\varphi \psi) & =B_{\alpha \beta}\left(\Xi_{j, \sigma}(\varphi \psi)\right), \\
W_{j}^{\prime} \circ B_{\alpha \beta}(\varphi \psi) & =B_{\alpha \beta}\left(W_{j}^{\prime} \circ(\varphi \psi)\right),
\end{aligned}
$$

where the notation $W_{j} \circ h\left(x, D_{x}\right)$ means the usual composition of (pseudo) differential operators.

Definition 4.3.3. Using the fields $W_{j}$ and $\Xi_{j}$, denote by $\left(\varphi_{j}^{(\prime)} \psi_{j}^{(\prime)}\right)^{(s)}$ any sum of $C^{s}$ operators of the form

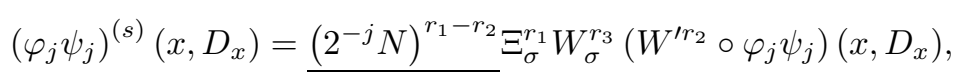

where $s=\left|r_{1}+r_{3}\right|,\left|r_{2}\right| \leq\left|r_{1}\right|, C$ is a universal constant and the operations $\Xi_{\sigma}$, $W_{\sigma}$ and $W^{\prime} \circ$ may occur in any order. In this sense equation (4.3.17) is a formal equation.

Proposition 4.3.2. For any multi-indices $a, b$ and for any $s$, such that $|a+b|+s \leq$ $2^{-j+1} N$, we have

$$
\left|\partial_{x}^{a} \partial_{\xi}^{b}\left(\varphi_{j} \psi_{j}\right)^{(s)}(x, \xi)\right| \leq C\left(C K_{j}\right)^{|a+b|+s}\left(2^{-j} N\right)^{|a|+s}\left(\frac{2^{-j} N}{|\xi|}\right)^{|b|}
$$

$j=0,1, \ldots$.

Proof. For the sake of brevity put $N_{j}=2^{-j} N$. The quantity on the left-hand side of (4.3.18) can be written as a sum of terms of the form

$$
\partial_{x}^{a} \partial_{\xi}^{b} N_{j}^{r_{1}-r_{2}} \Xi_{\sigma}^{r_{1}} W_{\sigma}^{r_{3}}\left(W^{\prime r_{2}} \circ\right)\left(\varphi_{j} \psi_{j}\right)\left(x, D_{x}\right) .
$$


Let us take a look at the symbol of this operator. Since $W^{\prime}$ has a symbol containing either $\xi_{j}$ or $x_{j} \xi_{n}$ we see that $W^{\prime r_{2}} \circ h$ has a symbol of the form

$$
\sum_{r_{2}^{\prime}+r_{2}^{\prime \prime}=r_{2}} \sum_{\ell \leq r_{2}^{\prime \prime}} a_{r_{2}^{\prime}, r_{2}^{\prime \prime}, \ell} x^{r_{2}^{\prime \prime}-\ell} \xi^{r_{2}^{\prime \prime}} \partial_{x}^{r_{2}^{\prime}} h
$$

so that if we apply $\Xi^{r_{1}}$ to this symbol and remark that $\Xi_{\sigma}$ means either a $\xi$ derivative or a $\xi$-derivative multiplied by an $x_{j}$, we obtain that

$$
\partial_{\xi}^{b} \Xi_{\sigma}^{r_{1}}\left(W^{\prime r_{2}} \circ\right) h=\sum_{\substack{r_{2}^{\prime}+r_{2}^{\prime \prime}=r_{2} \\ \ell \leq r_{2}^{\prime \prime}}} \sum_{\rho_{1}, s_{2}} a_{r_{2}^{\prime}, r_{2}^{\prime \prime}, \ell, \rho_{1}, s_{2}} \xi^{r_{2}^{\prime \prime}-s_{2}} \partial_{\xi}^{r_{1}-s_{2}} \partial_{x}^{r_{2}^{\prime}} h,
$$

provided $s_{2} \leq r_{1}+b, s_{2} \leq r_{2}^{\prime \prime}$. Analogously, applying $\partial_{x}^{a} W_{\sigma}^{r_{3}}$ to the above symbol, we obtain

$$
\begin{aligned}
\partial_{x}^{a} W_{\sigma}^{r_{3}} \partial_{\xi}^{b} \Xi_{\sigma}^{r_{1}}\left(W^{\prime r_{2}} \circ\right) h & =C \sum_{\substack{r_{2}^{\prime}+r_{2}^{\prime \prime}=r_{2} \\
\ell \leq r_{2}^{\prime \prime}}} \sum_{\rho_{1}, s_{2}} \sum_{\rho_{3}, s_{1}} x^{r_{1}+r_{3}+r_{2}^{\prime \prime}-\ell-s_{1}-\rho_{1}-\rho_{3}} \xi_{2}^{r_{2}^{\prime \prime}-s_{2}} \partial_{\xi}^{r_{1}+b-s_{2}} \partial_{x}^{r_{2}^{\prime}+a+r_{3}-s_{1}} h,
\end{aligned}
$$

where $s_{1} \leq r_{2}^{\prime}+r_{3}+a$ and $s_{2}$ has the same bounds as above. Roughly speaking we may say that the quantity in the left hand side of (4.3.18) can be written as a sum of terms of the form

$$
N_{j}^{r_{1}-r_{2}} x^{\left(r_{1}+r_{3}+r_{2}^{\prime \prime}-s_{1}\right) \leq} \xi^{r_{2}^{\prime \prime}-s_{2}} \partial_{\xi}^{r_{1}+b-s_{2}} \partial_{x}^{r_{2}^{\prime}+a+r_{3}-s_{1}}\left(\varphi_{j} \psi_{j}\right),
$$

where $s_{2} \leq r_{1}+b, s_{2} \leq r_{2}^{\prime \prime}, s_{1} \leq r_{2}^{\prime}+r_{3}+a, r_{2}^{\prime}+r_{2}^{\prime \prime}=r_{2},\left|r_{2}\right| \leq\left|r_{1}\right|$ and $(r) \leq=$ integer that can be bounded by $r$.

Now taking into account (3.1.3)-(3.1.6), since $r_{2} \leq r_{1},|\xi| \geq N_{j}$ on the support of $\psi_{j}$, and $|\xi| \leq 2 N_{j}$ on the support of any derivative of $\psi_{j}$, we obtain that the latter term can be estimated by

$$
K_{j}^{\left|r_{1}+b-s_{2}+r_{2}^{\prime}+a+r_{3}-s_{1}\right|}\left(\frac{N_{j}}{|\xi|}\right)^{r_{1}+b-r_{2}^{\prime \prime}} N_{j}^{\left|r_{1}+r_{3}+a\right|}
$$

and this can be estimated by

$$
K_{j}^{|a+b|+s} N_{j}^{|a|+s}\left(\frac{N_{j}}{|\xi|}\right)^{|b|}
$$

thus proving the assertion.

Corollary 4.3.2. Let $s \leq 2 N, w \in L^{2}\left(\mathbb{R}^{n}\right)$; then

$$
\left\|\left(\varphi_{j} \psi_{j}\right)^{(s)}\left(x, D_{x}\right) w\right\|_{L^{2}} \leq C\left(C K_{j}\right)^{s+n+1} N_{j}^{s+n+1}\|w\|_{L^{2}} .
$$

Proof. By Theorem 18.1.11' in [7], if $H\left(x, D_{x}\right)$ is a pseudo-differential operator of order 0 whose symbol has $x$-support contained in a fixed compact set $\bar{\Omega}$ of $\mathbb{R}^{n}$, we have

$$
\left\|H\left(x, D_{x}\right)\right\|_{L^{2} \rightarrow L^{2}} \leq C \operatorname{vol}(\Omega) \sup _{\substack{x, \xi \\|\rho| \leq n+1}}\left|\partial_{x}^{\rho} h(x, \xi)\right| .
$$

This fact, together with the preceding proposition, gives the assertion. 


\section{The A Priori Estimate}

5.1. Preparations. Using (2.4), let us write.

$$
P(x, \xi)=\sum_{|\alpha| \leq 2} a_{\alpha}(x, \xi) Z^{\alpha}+b(x, \xi) T
$$

and

$$
P_{\left(x_{0}, \xi_{0}\right)}(x, \xi)=\sum_{|\alpha| \leq 2} a_{\alpha}\left(x_{0}, \xi_{0}\right) Z^{\alpha}+b\left(x_{0}, \xi_{0}\right) T .
$$

Then by (2.7) we have the a priori estimate with frozen coefficients:

$$
\sum_{|J| \leq 2}{ }^{\prime}\left\|Z^{J} v\right\|^{2}+\|v\|_{1}^{2} \leq C\left(\left\|P_{\left(x_{0}, \xi_{0}\right)}(x, D) v\right\|^{2}+\|v\|^{2}\right)
$$

where $\sum_{|J| \leq 2}^{\prime}\left\|Z^{J} v\right\|^{2}=\sum_{|J| \leq 2}\left(\left\|X^{J} v\right\|^{2}+\left\|Y^{J} v\right\|^{2}\right)$. We may also allow $x$ to vary in a suitable small open set, e.g. supposing $v \in C_{0}^{\infty}\left(\mathbb{R}^{n}\right)$ with small support:

$$
\sum_{|J| \leq 2}{ }^{\prime}\left\|Z^{J} v\right\|^{2}+\|v\|_{1}^{2} \leq C\left(\left\|P_{\left(x_{0}, \xi_{0}\right)}(x, D) v\right\|^{2}+\|v\|^{2}\right)
$$

where

$$
P_{\left(x, \xi_{0}\right)}(x, \xi)=\sum_{|\alpha| \leq 2} a_{\alpha}\left(x, \xi_{0}\right) Z^{\alpha}+b\left(x, \xi_{0}\right) T .
$$

We have

$$
\begin{aligned}
\left\|P_{\left(x, \xi_{0}\right)}(x, D) v\right\| & \leq\|P(x, D) v\|+\left\|\left(P-P_{x, \xi_{O}}(x, D) \psi_{i}^{\prime}(x, D)\right) v\right\| \\
& \leq \varepsilon \sum_{|J| \leq 2}{ }^{\prime}\left\|Z^{J} v\right\|+C \sum_{|J| \leq 2}{ }^{\prime}\left\|Z^{J}\left(1-\psi_{i}^{\prime}(D)\right) v\right\|+\|P(x, D)\|,
\end{aligned}
$$

where we used the fact that $a_{\alpha}\left(x, \xi_{0}\right)-a_{\alpha}(x, D)$ has small $L^{2}$ norm when applied to $\psi_{i}^{\prime}(D) v$, provided cone supp $\psi_{i}^{\prime}(\xi)$ is small enough.

Our purpose will be to deal with a function $r$ of the form

$$
v=Z^{I^{\prime}} T^{q}\left(T^{p}\right)_{\left(\varphi_{i} \psi_{i}\right)^{(r)}} u .
$$

Lemma 5.1.1. Let $r \leq 2 N_{i}, N_{i}=N 2^{-i},\left|I^{\prime}\right|+q+p \leq N_{i}$ and $w \in L^{2}(\Omega)$. Then

$$
\begin{gathered}
\sum_{|J| \leq 2}\left\|Z^{J}\left(1-\psi_{i}^{\prime}(D)\right) Z^{I^{\prime}} T^{q}\left(T^{p}\right)_{\left(\varphi_{i} \psi_{i}\right)(r)} w\right\|_{L^{2}(\Omega)} \\
\leq C K_{i}^{N_{i}} N_{i}^{\left|I^{\prime}\right|+p+q+r}\|w\|_{L^{2}(\Omega)} .
\end{gathered}
$$

Proof. By Proposition A.4 we may write:

$$
\begin{gathered}
Z^{J}\left(1-\psi_{i}^{\prime}(D)\right) Z^{I^{\prime}} T^{q}\left(T^{p}\right)_{\left(\varphi_{i} \psi_{i}\right)^{(r)}} w \\
=\frac{C^{|J|+\left|I^{\prime}\right|+p} N_{i}^{\ell}\left(1-\psi_{i}^{\prime}(D)\right)^{\left(J^{\prime}\right)} x^{\left|I^{\prime}+J^{\prime \prime}\right|+p-\ell}}{\cdot D^{\left|I^{\prime}+J^{\prime \prime}\right|+q+p-\ell} \frac{1}{p^{\prime} !}\left(\varphi_{i} \psi_{i}\right)^{\left(s+p^{\prime}\right)} w,}
\end{gathered}
$$


where $J^{\prime}+J^{\prime \prime}=J, p^{\prime} \leq p, \ell \leq\left|I^{\prime}+J^{\prime \prime}\right|+|p|$ (note that the increase of $s$ has been obtained from the definition of $\left(\varphi_{i} \psi_{i}\right)^{(r)}$, the definition of $\left(T^{p}\right)_{\left(\varphi_{i} \psi_{i}\right)^{(r)}}$ and equation (4.3.12). The last quantity equals

$$
\begin{gathered}
\frac{C^{|J|+\left|I^{\prime}\right|+p} N_{i}^{\ell}}{\ell}\left(1-\psi_{i}^{\prime}(D)\right)^{\left(J^{\prime}\right)} x^{\left|I^{\prime}+J^{\prime \prime}\right|+p-\ell} \cdot D^{\left|I^{\prime}+J^{\prime \prime}\right|+q+p-\ell} \frac{1}{p^{\prime} !}\left(\varphi_{i} \psi_{i}\right)^{\left(s+p^{\prime}\right)} w \\
=C^{\left|J+I^{\prime}\right|+P} N_{i}^{\ell} x^{\left|I^{\prime}+J^{\prime \prime}\right|+p-\ell-\ell^{\prime}} D^{\left|I^{\prime}+J^{\prime \prime}\right|+q+p-\ell} \frac{1}{p^{\prime} !} \\
\cdot\left[\left(1-\psi_{i}^{\prime}\right)(D)^{\left(J^{\prime}+\ell^{\prime}\right)},\left(\varphi_{i} \psi_{i}\right)^{\left(s+p^{\prime}\right)}\right] w,
\end{gathered}
$$

where $\ell^{\prime} \leq\left|I^{\prime}+J^{\prime \prime}\right|+p-\ell$. Thus in order to prove the lemma it suffices to prove that

$$
\left\|D_{x}^{\tau_{1}}\left[\psi_{i}^{\left(\tau_{2}\right)},\left(\varphi_{i} \psi_{i}\right)^{\left(\tau_{3}\right)}\right]\right\| \leq K_{i}^{N_{i}} N_{i}^{\tau_{1}+\tau_{3}}\|u\|,
$$

when $\left|\tau_{1}+\tau_{2}+\tau_{3}\right| \leq 2 N_{i}$. Since $\psi_{i}^{\prime}$ is a function of $\xi$ only and due to the definition of the conical support of $\psi_{i}, \psi_{i}^{\prime}$ we must actually estimate

$$
\left\|D_{x}^{\tau_{1}}\left[\psi_{i}^{\prime\left(\tau_{2}\right)}\left(\varphi_{i} \psi_{i}\right)^{\left(\tau_{3}\right)}-\sum_{|\beta|<N_{i}} \frac{1}{\beta !}\left(\varphi_{i} \psi_{i}\right)^{\left(\tau_{3}+\beta\right)} \psi_{i}^{\prime\left(\tau_{2}+\beta\right)}\right] u\right\| .
$$

This is the same quantity of Lemma A.1, where now cone supp $f(x, \xi)$ has compact cosphere sections $\left(f=\psi_{i}^{\prime}\right)$. Using the notation of Lemma A.1, we remark that

$$
\int\left|r_{c_{1}}(x, \eta, \xi)\right| d \eta \leq \frac{C}{M !} \sup \left|f_{(\varepsilon)}^{\left(a_{2}\right)}(x, \sigma) \sigma^{a_{1}+b_{1}-c_{1}}\right| \mid\left(h^{\left(\varepsilon+b_{2}+c_{1}\right)}(\eta, \xi)\right) \hat{\mid}
$$

the supremum being taken over all $\varepsilon,|\varepsilon|=M, a_{1}+a_{2}=a,\left|b_{1}+b_{2}\right|=|b|+n+1$, $c_{1} \leq a_{1}+b_{1}$.

If $f$ has compact $x$-support or if $f$ is a function of $\xi$ only, using again the notation of Lemma A.1, we obtain that

$$
\begin{aligned}
& \left|D_{x}^{a}\left(F \circ H-\{F \circ H\}_{M}\right) D_{x}^{b} w\right| \\
& \leq \frac{C^{|a+b|+n}}{M !} \sup _{\substack{|\xi|=M \\
\left|b_{1}+b_{2}\right|=|b|+n+1 \\
c_{1} \leq a_{1}+b_{1} \\
a_{1}+a_{2}=a}}\left|f_{(\varepsilon)}^{\left(a_{2}\right)}(x, \sigma) \sigma^{a_{1}+b_{1}-c_{1}}\right|\left|\left(h^{\left(\varepsilon+b_{2}+c_{1}\right)}(\eta, \xi)\right)\right|\|w\| .
\end{aligned}
$$

Applying (5.1.10) to (5.1.9) and taking into account the properties (3.1.1)-(3.1.8), we get that (5.1.9) can be estimated by $K_{i}^{N_{i}} N_{i}^{\left(\tau_{1}+\tau_{3}\right)}\|u\|$, and this ends the proof of the Lemma.

Applying Lemma 5.1.1 and the a priori inequality (5.1.3) allows us to deduce

$$
\begin{aligned}
& \sum_{|J| \leq 2}{ }^{\prime}\left\|Z^{J} Z^{I^{\prime}} T^{q}\left(T^{p}\right)_{\left(\varphi_{i} \psi_{i}\right)^{(r)}} u\right\|_{L^{2}(\Omega)} \\
& \quad \leq C\left(\left\|P_{i} Z^{I^{\prime}} T^{q}\left(T^{p}\right)_{\left(\varphi_{i} \psi_{i}\right)^{(r)}} u\right\|_{L^{2}(\Omega)}+\left\|Z^{I^{\prime}} T^{q}\left(T^{p}\right)_{\left(\varphi_{i} \psi_{i}\right)^{(r)}} u\right\|_{L^{2}(\Omega)}\right. \\
& \\
& \left.\quad+K_{i}^{N_{i}} N_{i}^{\left|I^{\prime}\right|+q+p+r}\|u\|_{L^{2}(\Omega)}\right)
\end{aligned}
$$


where $P_{i}$ is defined by

$$
\begin{aligned}
P_{i} & \left(x, D_{x}\right) \\
& =\sum_{|\alpha| \leq 2} \Phi_{i}(x) a_{\alpha}\left(x, D_{x}\right) \psi_{i}^{\prime}(D) Z^{\alpha}+\Phi_{i}(x) b\left(x, D_{x}\right) \psi_{i}^{\prime}(D) T \\
& =\sum_{|\alpha| \leq 2} \tilde{a}_{\alpha i}\left(x, D_{x}\right) Z^{\alpha}+\tilde{b}_{i}\left(x, D_{x}\right) T
\end{aligned}
$$

where $\Phi_{i}(x) \equiv 1$ in a neighborhood of $\bar{\Omega}, \Phi_{i} \in C_{0}^{\infty}\left(\Omega^{\prime}\right), \Omega \subset \subset \Omega^{\prime}$ and $\left|D^{\alpha} \Phi_{i}(x)\right| \leq$ $\left(C N_{i}\right)^{|\alpha|}$ if $|\alpha| \leq 3 N_{i}$ (see e.g. (3.1.5)). We point out that the introduction of such a function $\Phi_{i}$ is always possible since the $L^{2}$ norms in (5.1.11) are actually $L^{2}(\Omega)$-norms.

5.2. The use of the a priori estimate. Let now $u \in C_{0}^{\infty}(\Omega)$. Assume that $i=0$ and apply (5.1.11) for $i=0$ with $q=0=r,\left|I^{\prime}\right|=0$ :

$$
\begin{aligned}
& \sum_{|J| \leq 2}\left\|Z^{J}\left(T^{p}\right)_{\varphi_{0} \psi_{0}} u\right\|_{L^{2}(\Omega)} \\
& \quad C \quad C\left(\left\|P_{0}\left(x, D_{x}\right)\left(T^{p}\right)_{\varphi_{0} \psi_{0}} u\right\|_{L^{2}(\Omega)}+\left\|\left(T^{p}\right)_{\varphi_{0} \psi_{0}} u\right\|_{L^{2}(\Omega)}\right. \\
& \left.\quad+K_{0}^{N_{0}} N_{0}^{p}\|u\|_{L^{2}(\Omega)}\right) .
\end{aligned}
$$

Our purpose is to iterate (5.2.1) in the following sense: we must estimate the term $\left\|P_{0}\left(T^{p}\right)_{\varphi_{0}} \psi_{0} u\right\|$. In order to do this we write

$$
P_{0}\left(T^{p}\right)_{\varphi_{0} \psi_{0}} u=\left(T^{p}\right)_{\varphi_{0} \psi_{0}} P_{0} u+\left[P_{0},\left(T^{p}\right)_{\varphi_{0} \psi_{0}}\right] u
$$

The first term is known and hence it will be a good term in our estimate; on the other hand the commutator generates a certain number of terms according to Proposition 4.2.1. In particular new $X^{\prime}$ 's and $Y^{\prime}$ 's appear causing the number of $T^{\prime}$ 's and the index $r$ to increase as $p$ decreases. Now in the final step of the first iteration there is a term with $p=0$; since basically a commutator of two $X^{\prime}$ 's generates a $T$ vector field, at this point $q$ may be as large as the original $p$ divided by 2 . We are then allowed to reboot another iteration procedure by introducing the next pair of cut off functions $\varphi_{1} \psi_{1}$ and use (5.1.11) over and over. Hence our main task will be to commute $P_{i}$ with $\left.Z^{I^{\prime}} T^{q}\left(T^{p}\right)_{\left(\varphi_{i} \psi_{i}\right)}\right)^{(r)}$.

We have

$$
\left[P_{i}, Z^{I^{\prime}} T^{q}\left(T^{p}\right)_{\left(\varphi_{i} \psi_{i}\right)^{(r)}}\right]=\left[P_{i}, Z^{I^{\prime}} T^{q}\right]\left(T^{p}\right)_{\left(\varphi_{i} \psi_{i}\right)^{(r)}}+Z^{I^{\prime}} T^{q}\left(T^{p}\right)_{\left(\varphi_{i} \psi_{i}\right)^{(r)}} .
$$

Recalling that, from (5.1.12),

$$
P_{i}(x, D)=\sum_{|\alpha| \leq 2} \tilde{a}_{\alpha i}(x, D) Z^{\alpha}+\tilde{b}_{i}(x, D) T
$$

we may write 


$$
\begin{aligned}
& {\left[P_{i}, Z^{I^{\prime}} T^{q}\left(T^{p}\right)_{\left(\varphi_{i} \psi_{i}\right)^{(r)}}\right]} \\
& =\sum_{|\alpha| \leq 2}\left[\left[\tilde{a}_{\alpha i}, Z^{I^{\prime}} T^{q}\right] Z^{\alpha}\left(T^{p}\right)_{\left(\varphi_{i} \psi_{i}\right)^{(r)}}\right. \\
& \quad+\tilde{a}_{\alpha i}\left[Z^{\alpha}, Z^{I^{\prime}}\right] T^{q}\left(T^{p}\right)_{\left(\varphi_{i} \psi_{i}\right)^{(r)}} \\
& \quad+Z^{I^{\prime}} T^{q}\left[\tilde{a}_{\alpha i},\left(T^{p}\right)_{\left(\varphi_{i} \psi_{i}\right)^{(r)}}\right] Z^{\alpha} \\
& \left.\quad+Z^{I^{\prime}} T^{q} \tilde{a}_{\alpha i}\left[Z^{\alpha},\left(T^{p}\right)_{\left(\varphi_{i} \psi_{i}\right)^{(r)}}\right]\right] \\
& \quad+\left[\tilde{b}_{i}, Z^{I^{\prime}} T^{q}\right] T\left(T^{p}\right)_{\left(\varphi_{i} \psi_{i}\right)^{(r)}}+\tilde{b}_{i}\left[T, Z^{I^{\prime}} T^{q}\right]\left(T^{p}\right)_{\left(\varphi_{i} \psi_{i}\right)^{(r)}} \\
& \quad+Z^{I^{\prime}} T^{q}\left[\tilde{b}_{i},\left(T^{p}\right)_{\left(\varphi_{i} \psi_{i}\right)^{(r)}}\right] T \\
& \quad+Z^{I^{\prime}} T^{q} \tilde{b}_{i}\left[T,\left(T^{p}\right)_{\left(\varphi_{i} \psi_{i}\right)^{(r)}}\right] \\
& =\sum_{|\alpha| \leq 2} \sum_{j=1}^{4} A_{\alpha j}^{(i)}+\sum_{j=1}^{4} B_{j}^{(i)}
\end{aligned}
$$

where $\tilde{a}_{\alpha}, \tilde{b}$ are defined in (5.1.12). We deal first with the $A$-terms; the $B^{\prime}$ 's will then be easy. First of all we point out that if $X$ is a fixed vector field we have the identity (easily proved by induction)

$$
\left[\tilde{a}_{\alpha i}, X^{k}\right]=\sum_{1 \leq k^{\prime} \leq k}\left(\begin{array}{c}
k \\
k^{\prime}
\end{array}\right) a d_{X}^{k^{\prime}} \tilde{a}_{\alpha i} X^{k-k^{\prime}}
$$

Iterating (5.2.3) and using the multi-index notation we have

$$
\begin{aligned}
A_{\alpha 1}^{(i)}= & -\sum_{\substack{\left|I^{\prime \prime}\right| \leq\left|I^{\prime}\right| \\
I^{\prime} \leq q \\
\left|I^{\prime \prime}\right|+q^{\prime} \geq 1\\
}}\left(\begin{array}{c}
\left|I^{\prime}\right| \\
\left|I^{\prime \prime}\right|
\end{array}\right)\left(\begin{array}{c}
q \\
q^{\prime}
\end{array}\right) \tilde{a}_{\alpha i}^{\left(\left|I^{\prime \prime}\right|+q^{\prime}\right)} \\
& \circ Z^{I^{\prime}-I^{\prime \prime}+\alpha} T^{q-q^{\prime}}\left(T^{p}\right)_{\left(\varphi_{i} \psi_{i}\right)^{(r)}},
\end{aligned}
$$

where we denote by

$$
\tilde{a}_{\alpha i}^{(s)}\left(x, D_{x}\right)=a d_{Z_{i_{1}}} \circ \cdots \circ a d_{Z_{i_{s}}}\left(\tilde{a}_{\alpha i}\left(x, D_{x}\right)\right)
$$

$Z_{i_{1}}, \ldots, Z_{i_{s}}$ being vector fields belonging to a "fixed" finite set of analytic vector fields (e.g. all the vector fields used until now, i.e. the $Z$ 's, the $W$ 's and the $\Xi$ 's, may build such a family). Next

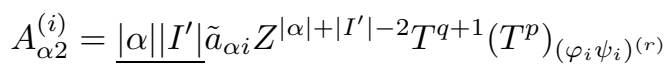

where this term is missing if $\left|I^{\prime}\right|=0$ and by $Z^{|\alpha|+\left|I^{\prime}\right|-2}$ we denote an expression of the form $Z^{\beta}$, with $|\beta|=|\alpha|+\left|I^{\prime}\right|-2$; note that this term is also missing if no commutator between $X^{\prime}$-type and $X^{\prime \prime}$-type field is involved. Furthermore using 
(4.2.8) and making again the same conventions as above we obtain:

$$
\begin{aligned}
A_{\alpha 4}^{(i)}= & \sum_{\ell=1}^{\left|\alpha^{\prime}\right|}(-1)^{\ell-1}\left(\begin{array}{c}
\left|\alpha^{\prime}\right| \\
\ell
\end{array}\right) Z^{I^{\prime}} T^{q} \tilde{a}_{\alpha i}\left(T^{p-\ell}\right)_{\left(\tilde{\varphi}_{i} \psi_{i}\right)^{(r+\ell)} \varphi^{\sharp\left(\alpha-\alpha^{\prime}\right)}} \\
= & \sum_{\ell=0}^{\left|\alpha^{\prime}\right|-1\left|\alpha^{\prime}\right|-\ell} \sum_{k=1} \underline{C}^{p-\ell} \frac{1}{(p-\ell) !} Z^{I^{\prime}} T^{q} \\
& \quad \tilde{a}_{\alpha i} X^{p+\left|\alpha^{\prime}\right|-\ell-k-1}\left(\tilde{\varphi}_{i} \psi_{i}\right)^{(r+p+k+1)} \varphi^{\sharp\left(\alpha-\alpha^{\prime}\right)} .
\end{aligned}
$$

We point out explicitly that in the above equation $Z^{\alpha}$ has been decomposed as $Y^{\alpha-\alpha^{\prime}} X^{\alpha^{\prime}}\left(X\right.$ equals either $X^{\prime}$ or $\left.X^{\prime \prime}\right)$ and the $Y$ vector fields act on $\left(T^{p}\right)_{\left(\varphi_{i} \psi_{i}\right)}$ according to (4.2.7) - see also Remark 4.2.2 - whereas we applied (4.2.8) only to the $X$ vector fields (see also (3.1.9)).

$$
\begin{aligned}
& \sum_{|\alpha| \leq 2}\left(A_{\alpha 1}^{(i)}+A_{\alpha 2}^{(i)}+A_{\alpha 4}^{(i)}\right)
\end{aligned}
$$

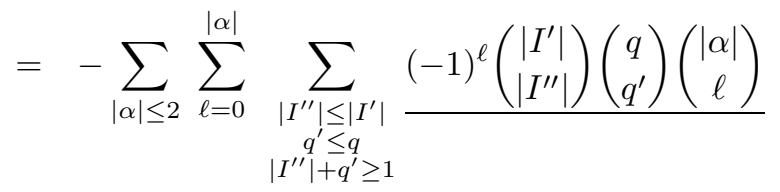

$$
\begin{aligned}
& \text {. } \tilde{a}_{\alpha i}^{\left(\left|I^{\prime \prime}\right|+q^{\prime}\right)} Z^{I^{\prime}-I^{\prime \prime}+\alpha} T^{q-q^{\prime}}\left(T^{p-\ell}\right)_{\left(\varphi_{i} \psi_{i}\right)^{(r+\ell)}}
\end{aligned}
$$

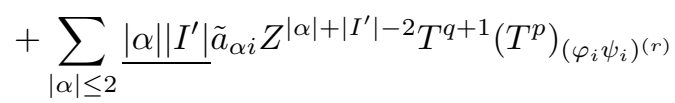

$$
\begin{aligned}
& +\sum_{\substack{|\alpha| \leq 2 \\
\alpha^{\prime} \leq \alpha}} \sum_{\ell=1}^{|\alpha|-1} \sum_{k=0}^{|\alpha|-\ell} \sum_{\substack{\left|I^{\prime \prime}\right| \leq\left|I^{\prime}\right| \\
q^{\prime} \leq q \\
\left|I^{\prime \prime}\right|+q^{\prime} \geq 1}} C^{p-\ell}\left(\begin{array}{c}
\left|I^{\prime}\right| \\
\left|I^{\prime \prime}\right|
\end{array}\right)\left(\begin{array}{c}
q \\
q^{\prime}
\end{array}\right) \frac{1}{(p-\ell) !} \\
& \tilde{a}_{\alpha i}^{\left(\left|I^{\prime \prime}\right|+q^{\prime}\right)} Z^{\left|I^{\prime}-I^{\prime \prime}\right|} X^{\left|\alpha^{\prime}\right|+p-\ell-k-1} T^{q-q^{\prime}}\left(\tilde{\varphi}_{i} \psi_{i}\right)^{(r+p+k+1)} \varphi^{\sharp\left(\alpha-\alpha^{\prime}\right)} .
\end{aligned}
$$

Proposition 5.2.1. Assume $\tilde{a}_{\alpha i}$ is a $G^{s}$ pseudo-differential operator of order zero and assume that $\tilde{a}_{\alpha i}^{(\ell)}$ entails only $x$-derivatives of the symbol $\tilde{a}_{\alpha i}^{(\ell)}(x, \xi)$. Then if $u \in L^{2}(\Omega) \cap \mathcal{E}^{\prime}(\Omega)$,

$$
\left\|\tilde{a}_{\alpha i}^{(\ell)}\left(x, D_{x}\right) u\right\|_{L^{2}(\Omega)} \leq C^{\ell+1} \ell !^{s}\|u\|_{L^{2}(\Omega)} .
$$

Here $s \geq 1$.

Proof. Since

$$
\left\|\tilde{a}_{\alpha i}^{(\ell)}\left(x, D_{x}\right) u\right\|_{L^{2}(\Omega)} \leq C \sup _{\substack{|\rho| \leq n+1 \\ \xi \in \mathbb{R}^{n}}}\left\|D_{x}^{\rho} \sigma\left(\tilde{a}_{\alpha i}^{(\ell)}\right)(x, \xi)\right\|_{L^{1}(\Omega)}\|u\|_{L^{2}(\Omega)},
$$

the conclusion follows at once from (3.4.1) and the definition of $\tilde{a}_{\alpha i}$ in (5.1.12). 
Assembling the estimates for the terms in (5.2.8) and (5.1.11) we obtain:

$$
\begin{aligned}
& \sup _{|J| \leq 2}\left\|Z^{J} Z^{I^{\prime}} T^{q}\left(T^{p}\right)_{\left(\varphi_{i} \psi_{i}\right)^{(r)}} u\right\|_{L^{2}(\Omega)} \\
& \leq C\left\{\left\|Z^{I^{\prime}} T^{q}\left(T^{p}\right)_{\left(\varphi_{i} \psi_{i}\right)^{(r)}} \Phi_{i} P u\right\|_{L^{2}(\Omega)}\right. \\
& +\left\|Z^{I^{\prime}} T^{q}\left(T^{p}\right)_{\left(\varphi_{i} \psi_{i}\right)(r)}\left(\Phi_{i} P-P_{i}\right) u\right\|_{L^{2}(\Omega)} \\
& +\left\|Z^{I^{\prime}} T^{q}\left(T^{p}\right)_{\left(\varphi_{i} \psi_{i}\right)^{(r)}} u\right\|_{L^{2}(\Omega)}+K_{i}^{N_{i}} N_{i}^{\left|I^{\prime}\right|+p+q+r}\|u\|_{L^{2}(\Omega)} \\
& +\sup _{\substack{|\alpha| \leq 2, \ell \leq 2 \\
\left|I^{\prime \prime}\right| \leq\left|I^{\prime}\right|}} C^{\left|I^{\prime \prime}\right|+q^{\prime}} N_{i}^{\left(\left|I^{\prime \prime}\right|+q^{\prime}\right) s} \\
& \begin{array}{c}
q^{\prime} \leq q \\
\left|I^{\prime \prime}\right|+q^{\prime} \geq 1
\end{array} \\
& \cdot\left\|Z^{I^{\prime}-I^{\prime \prime}+\alpha} T^{q-q^{\prime}}\left(T^{p-\ell}\right)_{\left(\varphi_{i} \psi_{i}\right)^{(r+\ell)}} u\right\|_{L^{2}(\Omega)} \\
& +\sup _{|\alpha| \leq 2}\left|I^{\prime}\right|\left\|Z^{\alpha+I^{\prime}-2} T^{q+1}\left(T^{p}\right)_{\left(\varphi_{i} \psi_{i}\right)^{(r)}} u\right\|_{L^{2}(\Omega)} \\
& +\sup _{\substack{|\alpha| \leq \ell, \alpha^{\prime} \leq \alpha \\
1 \leq \ell \leq|\alpha|-1 \\
0 \leq k \leq|\alpha|-\ell \\
q^{\prime} \leq q,\left|I^{\prime \prime}\right| \leq\left|I^{\prime}\right| \\
\left|I^{\prime \prime}\right|+q^{\prime} \geq 1}} C^{\left|I^{\prime \prime}\right|+q^{\prime}} N_{i}^{\left(\left|I^{\prime \prime}\right|+q^{\prime}\right) s} \frac{1}{(p-\ell) !} \\
& \cdot\left\|Z^{\left|I^{\prime}-I^{\prime \prime}\right|} X^{\left|\alpha^{\prime}\right|+p-\ell-k-1} T^{q-q^{\prime}}\left(\varphi_{i} \psi_{i}\right)^{(r+p+k+1)} \varphi^{\#\left(\alpha-\alpha^{\prime}\right)} u\right\|_{L^{2}(\Omega)} \\
& \left.+\sup _{|\alpha| \leq 2}\left\|A_{\alpha 3}^{(i)} u\right\|_{L^{2}(\Omega)}\right\}
\end{aligned}
$$

for $\left|I^{\prime}\right|+p+q+2 \leq N_{i}$.

Lemma 5.2.1. Let $\left|I^{\prime}\right|+p+q+2 \leq N_{i}, r \leq 2 N_{i}$, then

$$
\begin{gathered}
\left\|Z^{I^{\prime}} T^{q}\left(T^{p}\right)_{\left(\varphi_{i} \psi_{i}\right)^{(r)}}\left(\Phi_{i} P-P_{i}\right) u\right\|_{L^{2}(\Omega)} \\
\quad \leq K_{i}^{r+N_{i}+2} N_{i}^{r+\left|I^{\prime}\right|+p+q}\|u\|_{L^{2}(\Omega)},
\end{gathered}
$$

$p, q, I^{\prime}$ and $r$ being defined as above.

Proof. Recalling formula (A.4) and remarking that $\psi_{i}^{\prime}(\xi) \equiv 1$ on the support of $\psi_{i}$, we may write that

$$
\begin{aligned}
& Z^{I^{\prime}} T^{q}\left(T^{p}\right)_{\left(\varphi_{i} \psi_{i}\right)^{(r)}}\left(\Phi_{i} P-P_{i}\right) \\
& =\sum_{\substack{r^{\prime}+\tilde{r} \leq\left|I^{\prime}\right|+p+q \\
r^{\prime \prime} \leq 2,|\alpha| \leq 2}} \frac{C^{\left|I^{\prime}\right|+p+q} N_{i}^{r^{\prime}+r^{\prime \prime}}}{} O\left(|x|^{\left|I^{\prime}\right|+p-r^{\prime}}\right) \frac{1}{p !}\left(\varphi_{i} \psi_{i}\right)^{(r+p+\tilde{r})} \\
& \quad \times D_{x}^{\left|I^{\prime}\right|+p+q-r^{\prime}-\tilde{r}}\left[\Phi_{i} a_{\alpha},\left(1-\psi_{i}^{\prime}\right)(D)\right] D_{x}^{|\alpha|-r^{\prime \prime}} O\left(|x|^{|\alpha|-r^{\prime \prime}}\right),
\end{aligned}
$$

where $D_{x}^{k}$ means a derivative with respect to $x$ of order $k$. The first order term in $T$ in the expression of $P$ receives a completely analogous treatment (even simpler!). Denoting by $\psi^{\prime \prime}(\xi)$ a cut-off symbol of order zero such that supp $\psi_{i}^{\prime \prime} \subset\left\{\psi_{i}^{\prime} \equiv 1\right\}$, 
$\psi_{i}^{\prime} \equiv 1$ on the support of $\psi_{i},\left|\psi_{i}^{\prime \prime}\right| \leq 1$, we easily see that, due to Corollary 4.3.2 it suffices to show that

$$
\begin{aligned}
& \left\|\psi_{i}^{\prime \prime}(D) D_{x}^{\tau_{1}}\left[\Phi_{i} a,\left(1-\psi_{i}^{\prime}(D)\right)\right] D_{x}^{\tau_{2}} w\right\|_{L^{2}(\Omega)} \\
& \quad \leq K_{i}^{N_{i}} N_{i}^{\left|\tau_{1}+\tau_{2}\right|}\|w\|_{L^{2}(\Omega)}
\end{aligned}
$$

whenever $\left|\tau_{1}+\tau_{2}\right| \leq 2 N_{i}, \tau_{1}, \tau_{2}$ suitable multi-indices. Note that since supp $\psi_{i}^{\prime \prime} \subset$ $\left\{\psi_{i}^{\prime} \equiv 1\right\}$, we have

$$
\begin{aligned}
\psi_{i}^{\prime \prime} D_{x}^{\tau_{1}} & {\left[\Phi_{i} a,\left(1-\psi_{i}^{\prime}(D)\right)\right] } \\
& =\psi_{i}^{\prime \prime} D_{x}^{\tau_{1}} \Phi_{i} a\left(1-\psi_{i}^{\prime}(D)\right) \\
& =D_{x}^{\tau_{1}}\left[\psi_{i}^{\prime \prime}, \Phi_{i} a\right]\left(1-\psi_{i}^{\prime}(D)\right) \\
& =D_{x}^{\tau_{1}}\left[\psi_{i}^{\prime \prime} \Phi_{i} a-\sum_{|\beta| \leq N_{i}} \frac{1}{\beta !} \operatorname{Op}\left(\psi_{i(\beta)}^{\prime \prime}\left(\Phi_{i} a\right)^{(\beta)}\right)\right]\left(1-\psi_{i}^{\prime}(D)\right) .
\end{aligned}
$$

As a consequence we must estimate

$$
\begin{aligned}
& \left\|D_{x}^{\tau_{1}}\left[\psi_{i}^{\prime \prime} \Phi_{i} a-\sum_{|\beta| \leq N_{i}} \frac{1}{\beta !} \mathrm{Op}\left(\psi_{i(\beta)}^{\prime \prime}\left(\Phi_{i} a\right)^{(\beta)}\right)\right]\left(1-\psi_{i}^{\prime}(D)\right) D_{x}^{\tau_{2}} w\right\|_{L^{2}(\Omega)} \\
& \quad \leq K_{i}^{N_{i}} N_{i}^{\left|\tau_{1}+\tau_{2}\right|}\|w\|_{L^{2}(\Omega)},
\end{aligned}
$$

and the estimate in (5.2.14) follows from Lemma A.1 arguing along the same lines of Lemma 5.1.1.

5.3. Estimate of the term containing $A_{\alpha 3}^{(i)}$. In this section we will estimate terms of the form $\left[G(x, D),\left(T^{p}\right)_{\left(\varphi_{i} \psi_{i}\right)(r)}\right]$, modeling those which build $A_{\alpha 3}^{(i)}$. But first we need a suitable formula for the commutator of two pseudo-differential operators.

Lemma 5.3.1. Let $G(x, D), H(x, D)$ be two pseudo-differential operators with symbol $g(x, \xi), h(x, \xi)$ respectively and let

$$
\begin{gathered}
g_{(\beta)}^{(\alpha)}(x, \xi)=\partial_{\xi}^{\alpha} D_{x}^{\beta} g(x, \xi), \\
\sigma\left(G_{(\beta)}^{(\alpha)}\right)(x, \xi)=g_{(\beta)}^{(\alpha)}(x, \xi),
\end{gathered}
$$

for any multi-indices $\alpha, \beta$. Also define, for a positive integer $M$,

$$
\sigma\left(\{G \circ H\}_{M}\right)=\sum_{0 \leq \delta \leq M} \frac{1}{\delta !} g^{(\delta)}(x, \xi) h_{(\delta)}(x, \xi),
$$

i.e.

$$
\{G \circ H\}_{M}=G \circ H-\left(G \circ H-\mathrm{Op}\left(\sum_{0 \leq \delta \leq M} \frac{1}{\delta !} g^{(\delta)}(x, \xi) h_{(\delta)}(x, \xi)\right)\right),
$$


where, as usual, $\operatorname{Op}(q(x, \xi))$ denotes the pseudo-differential operator with symbol $q(x, \xi)$. Then for any $M$ non-negative integer,

$$
\{G \circ H\}_{M}-\{H \circ M\}_{M}=\sum_{1 \leq|\alpha+\beta| \leq M} \frac{(-1)^{|\beta|}}{\alpha ! \beta !}\left\{H_{(\beta)}^{(\alpha)} \circ G_{(\beta)}^{(\alpha)}\right\}_{M-|\alpha+\beta|} .
$$

Corollary 5.3.1. For any $M$ we have

$$
[G, H]=\sum_{1 \leq|\alpha+\beta| \leq M} \frac{(-1)^{|\beta|}}{\alpha ! \beta !} H_{(\beta)}^{(\alpha)} \circ G_{(\beta)}^{(\alpha)}+R_{[G, H], M}
$$

where

$$
\begin{aligned}
& R_{[G, H], M}=[G, H]-\left(\{G \circ H\}_{M}-\{H \circ M\}_{M}\right) \\
& \quad=\sum_{1 \leq|\alpha+\beta| \leq M} \frac{(-1)^{|\beta|}}{\alpha ! \beta !}\left(\left\{H_{(\beta)}^{(\alpha)} \circ G_{(\beta)}^{(\alpha)}\right\}_{M-|\alpha+\beta|}-H_{(\beta)}^{(\alpha)} \circ G_{(\beta)}^{(\alpha)}\right) .
\end{aligned}
$$

Corollary 5.3.1 is just a restatement of Lemma 5.3.1.

Proof of Lemma 5.3.1. By the general calculus we have

$$
\sigma([G, H]) \sim \sum_{|\gamma| \geq 1} \frac{1}{\delta !}\left(g^{(\delta)} h_{(\delta)}-h^{(\delta)} g_{(\delta)}\right) .
$$

Using the identity

$$
-1=\sum_{\substack{|\delta| \geq 1 \\
\gamma \leq|\delta|}}(-1)^{|\gamma|}\left(\begin{array}{l}
\delta \\
\gamma
\end{array}\right)
$$

we obtain that

$$
-\frac{1}{\delta !} h^{(\delta)} g_{(\delta)}=\sum_{1 \leq|\gamma| \leq|\delta|} \frac{(-1)^{|\gamma|}}{\gamma !(\delta-\gamma) !} h^{(\gamma)(\delta-\gamma)} g_{(\gamma)(\delta-\gamma)},
$$

so that

$$
-\sum_{1 \leq|\delta| \leq M} \frac{1}{\delta !} h^{(\delta)} g_{(\delta)}=\sum_{1 \leq|\gamma| \leq M} \sigma\left(\left\{H^{(\gamma)} \circ G_{(\gamma)}\right\}_{M-|\gamma|}\right) .
$$

Since

$$
h_{(\delta)} g^{(\delta)}=\sum_{0 \leq|\beta| \leq M-|\delta|} \frac{1}{\beta !} h^{(\delta)} g_{(\delta)}-\sum_{1 \leq|\beta| \leq M-|\delta|} \frac{1}{\delta !} h^{(\delta)} g_{(\delta)},
$$

using the same identity on the second term we obtain

$$
\begin{aligned}
& \sum_{1 \leq|\delta| \leq M} \frac{1}{\delta !} g^{(\delta)} h_{(\delta)}=\sum_{1 \leq|\delta| \leq M} \frac{1}{\delta !} \sigma\left(\left\{H_{(\delta)} \circ G^{(\delta)}\right\}_{M-|\delta|}\right) \\
& +\sum_{1 \leq|\delta| \leq M} \sum_{1 \leq|\gamma| \leq M-|\delta|} \frac{(-1)^{|\gamma|}}{\delta ! \gamma !} \sigma\left(\left\{H_{(\delta)}^{(\gamma)} \circ G_{(\gamma)}^{(\delta)}\right\}_{M-|\delta|}\right),
\end{aligned}
$$

which proves the lemma. 
Our purpose is now to give an estimate of the last term in (5.2.10). We use the convention that $\tilde{H}(x, D)$ denotes the operator

$$
\tilde{H}(x, D)=\Phi_{i}(x) H(x, D) \Psi_{i}^{\prime}(D),
$$

where $\varphi_{i} \equiv 1$ near $\bar{\Omega}$ and $\left|D^{\alpha} \Phi_{i}\right| \leq\left(C N_{i}\right)^{|\alpha|}$, for $|\alpha| \leq N_{i}$. It will also be useful to make a small change in the previous notation; namely we set

$$
\left(T^{p}\right)_{\varphi \psi}=\sum_{|\alpha+\beta| \leq p} \frac{(-1)^{|\alpha|}}{\alpha ! \beta !} A_{\alpha, \beta}^{(p)} \cdot B_{\alpha, \beta}^{(p)},
$$

where

$$
\begin{gathered}
A_{\alpha, \beta}^{(p)}=X^{\prime \prime \alpha} X^{\prime \beta} T^{p-|\alpha+\beta|} \\
B_{\alpha, \beta}^{(p)}=a d_{X^{\prime}}^{\alpha} a d_{X^{\prime \prime}}^{\beta}(\varphi \psi)=B_{\alpha, \beta}(\varphi \psi)=B_{\alpha, \beta} .
\end{gathered}
$$

Using the general formula

$$
[A B, \tilde{G}]=A[B, \tilde{G}]+[A, \tilde{G}] B
$$

and Corollary 5.3.1, we may write

$$
\begin{aligned}
& {\left[\left(T^{p}\right)_{\left(\varphi_{i} \psi_{i}\right)^{(r)}}, \tilde{G}\right]=R_{\left[\left(T^{p}\right)_{\left(\varphi_{i} \psi_{i}\right)}(r), \tilde{G}\right], N_{i}}} \\
& \begin{array}{l}
+\sum_{\substack{|\gamma+\delta| \leq N_{i} \\
\alpha^{\prime} \leq \alpha, \beta^{\prime} \leq \beta \\
\tau+|\alpha+\beta| \leq p \\
1 \leq|\gamma+\delta|+\left|\alpha^{\prime}+\beta^{\prime}\right|+\tau}}(-1)^{|\alpha|}\left(\begin{array}{c}
A \\
\beta^{\prime}
\end{array}\right)\left(\begin{array}{c}
\beta \\
\beta^{\prime}
\end{array}\right)\left(\begin{array}{c}
p-|\alpha+\beta| \\
\tau
\end{array}\right) \tilde{G}_{\left(\alpha^{\prime}, \beta^{\prime}, \tau, \delta\right)}^{(\gamma)} \\
\times A_{\alpha-\alpha^{\prime}, \beta-\beta^{\prime}}^{\left(p-\left|\alpha^{\prime}+\beta^{\prime}\right|-\tau\right)} B_{\alpha, \beta}\left(\left(\varphi_{i} \psi_{i}\right)^{(r)}\right)_{(\gamma)}^{(\delta)} \frac{1}{\alpha ! \beta ! \gamma ! \delta !} \\
R_{\left[\left(T^{p}\right)_{\left(\varphi_{i} \psi_{i}\right)}^{(r)}, \tilde{G}\right], N_{i}}+\Sigma_{r, i, \tilde{G}}^{1}+\Sigma_{r, i, \tilde{G}}^{2},
\end{array}
\end{aligned}
$$

where

$$
\begin{aligned}
& R_{\left[\left(T^{p}\right)_{\left(\varphi_{i} \psi_{i}\right)}^{(r)}, \tilde{G}\right], N_{i}}=\sum_{|\alpha+\beta| \leq p} \frac{(-1)^{|\alpha|}}{\alpha ! \beta !} \\
& \cdot\left[A_{\alpha, \beta}^{(p)}\left(B_{\alpha, \beta}^{(p)} \tilde{G}-\left\{B_{\alpha, \beta}^{(p)} \circ \tilde{G}\right\}_{N_{i}}\right)\right. \\
& -A_{\alpha, \beta}^{(p)}\left(\tilde{G} B_{\alpha, \beta}^{(p)}-\left\{\tilde{G} \circ B_{\alpha, \beta}^{(p)}\right\}_{N_{i}}\right) \\
& +A_{\alpha, \beta}^{(p)} \sum_{1 \leq|\lambda+\mu| \leq N_{i}} \frac{(-1)^{|\lambda|}}{\lambda ! \mu !} \\
& \left.\quad \times\left(\left\{\tilde{G}_{(\mu)}^{(\lambda)} \circ B_{\alpha, \beta(\lambda)}^{(p)(\mu)}\right\}_{N_{i}-|\lambda+\mu|}-\tilde{G}_{(\mu)}^{(\lambda)} \circ B_{\alpha, \beta(\lambda)}^{(p)(\mu)}\right)\right], \\
& \tilde{G}_{\left(\alpha^{\prime}, \beta^{\prime}, \tau, \delta\right)}^{(\gamma)}(x, D)=a d_{X^{\prime \prime}}^{\alpha^{\prime}} a d_{X^{\prime}}^{\beta^{\prime}} a d_{T}^{\tau}\left(\tilde{G}_{(\delta)}^{(\gamma)}(x, D)\right)
\end{aligned}
$$

and where $\Sigma_{r, i, \tilde{G}}^{1}$ and $\Sigma_{r, i, \tilde{G}}^{2}$ denote the sums over $|\gamma+\delta| \leq p-\left|\alpha^{\prime}+\beta^{\prime}\right|-\tau$ and $|\gamma+\delta|>p-\left|\alpha^{\prime}+\beta^{\prime}\right|-\tau$ respectively. Here $\tilde{G}_{\left(\alpha^{\prime}, \beta^{\prime}, \tau, \delta\right)}^{(\gamma)}$ has order $-|\gamma|$ and $B_{\alpha, \beta}\left(\left(\varphi_{i} \psi_{i}\right)^{(r)}\right)_{(\gamma)}^{(\delta)}$ has order $-|\delta|$. 
In $\Sigma_{r, i, \tilde{G}}^{1}$ we want to decrease the number of derivatives in $A_{\alpha-\alpha^{\prime}, \beta-\beta^{\prime}}^{\left(p-\left|\alpha^{\prime}+\beta^{\prime}\right|-\tau\right)}$ in order to bring $\tilde{G}_{\left(\alpha^{\prime}, \beta^{\prime}, \tau, \delta\right)}^{(\gamma)}$ and $B_{\alpha, \beta}\left(\left(\varphi_{i} \psi_{i}\right)^{(r)}\right)_{(\gamma)}^{(\delta)}$ up to order zero. When possible we do this exploiting exclusively powers of $T$ (grouping these terms in $E_{r, i, \tilde{G}}^{1}$ ) and with a mixture of $T$ and $X$ derivatives, otherwise (grouping these terms in $E_{r, i, \tilde{G}}^{2}$ )

$$
\Sigma_{r, i, \tilde{G}}^{1}=E_{r, i, \tilde{G}}^{1}+E_{r, i, \tilde{G}}^{2},
$$

where

$$
\begin{aligned}
E_{r, i, \tilde{G}}^{1}= & \sum_{\substack{|\gamma+\delta| \leq p-\left|\alpha^{\prime}+\beta^{\prime}\right|-\tau \\
|\gamma+\delta|+\mid \alpha^{\prime}+\beta^{\prime}+\tau \geq 1 \\
\alpha^{\prime} \leq \alpha, \beta^{\prime} \leq \beta}}(-1)^{|\alpha|}\left(\begin{array}{c}
\alpha \\
\alpha^{\prime}
\end{array}\right)\left(\begin{array}{c}
\beta \\
\beta^{\prime}
\end{array}\right)\left(\begin{array}{c}
p-|\alpha+\beta| \\
\tau
\end{array}\right) \\
& \cdot \tilde{G}_{\left(\alpha^{\prime}, \beta^{\prime}, \tau, \delta\right)}^{(\gamma)} T^{|\gamma|} A_{\alpha-\alpha^{\prime}, \beta-\beta^{\prime}}^{\left(p-\left|\alpha^{\prime}+\beta^{\prime}\right|-\tau-|\gamma+\delta|\right)} T^{|\delta|} \cdot B_{\alpha, \beta}\left(\left(\varphi_{i} \psi_{i}\right)^{(r)}\right)_{(\gamma)}^{(\delta)} \frac{1}{\alpha ! \beta ! \delta ! \gamma !}
\end{aligned}
$$

and

$$
\begin{aligned}
E_{r, i, \tilde{G}}^{2}= & \sum_{\substack{|\gamma+\delta| \leq p-\left|\alpha^{\prime}+\beta^{\prime}\right|-\tau \\
\left|\gamma+\delta+\alpha^{\prime}+\beta^{\prime}\right|+\tau \geq 1 \\
\alpha^{\prime} \leq \alpha, \beta^{\prime} \leq \beta}} \frac{(-1)^{|\alpha|}}{\alpha ! \beta ! \gamma ! \delta !}\left(\begin{array}{c}
\alpha \\
\alpha^{\prime}
\end{array}\right)\left(\begin{array}{c}
\beta \\
\beta^{\prime}
\end{array}\right)\left(\begin{array}{c}
p-|\alpha+\beta| \\
\tau
\end{array}\right) \\
& \cdot \tilde{G}_{\left(\alpha^{\prime}, \beta^{\prime}, \tau, \delta\right)}^{(\gamma)} Z^{|\gamma|} X^{\left(p-\left|\alpha^{\prime}+\beta^{\prime}\right|-\tau-|\gamma+\delta|\right)} Z^{|\delta|} \cdot B_{\alpha, \beta}\left(\left(\varphi_{i} \psi_{i}\right)^{(r)}\right)_{(\gamma)}^{(\delta)}
\end{aligned}
$$

where in (5.3.16) $Z$ means either an $X$ or a $T$ derivative. That is, if there are any $T$ vector fields remaining after bringing $\tilde{G}_{(\cdot)}^{(\cdot)}$ or $B_{\alpha, \beta(\cdot)}^{(\cdot)}$ up to have order zero in $\Sigma_{r, i, \tilde{G}}^{1}$, they appear explicitly in expressions containing $A$ or $B$.

As for $\Sigma_{r, i, \tilde{G}}^{2}$ we recall that we actually want to estimate the norm of

$$
V^{I} T^{q} \Sigma_{r, i, \tilde{G}}^{2} V^{K}
$$

applied to $u$. Our strategy will be to fully exhaust $A_{\alpha-\alpha^{\prime}, \beta-\beta^{\prime}}^{\left(p-\mid \alpha^{\prime}+\beta^{\prime}\right)}$ and bring in additional derivatives as well from the vector fields preceding and following $\Sigma_{r, i, \tilde{G}}^{2}$. Here, with a change in the notation used in the preceding sections, we denoted by $V$ either a derivative along the $X$ (i.e. $X^{\prime}, X^{\prime \prime}$ ) direction or along the $Y$ direction.

Thus we may write

$$
\begin{aligned}
& V^{I} T^{q} \Sigma_{r, i, \tilde{G}}^{2} V^{k} \\
& \quad=\underline{C^{N_{i}}} \tilde{G}_{\left(\alpha^{\prime}, \beta^{\prime}, \tau, \delta, s_{1}\right)}^{(\gamma)} Z^{s_{2}} Z^{s_{3}} Z^{s_{4}} a d_{Z}^{s_{5}}\left(B_{\alpha \beta}\left(\left(\varphi_{i} \psi_{i}\right)^{(r)}\right)_{(\gamma)}^{(\delta)}\right)
\end{aligned}
$$

where $\sum_{i=1}^{5} s_{i}=|I+K|+p+q-\left|\alpha^{\prime}+\beta^{\prime}\right|-\tau$, and, unless $s_{3}=0$, we may take $\left|s_{2}\right|=|\gamma|,\left|s_{4}\right|=|\delta|$. In any case $\left|s_{2}\right| \leq|\gamma|,\left|s_{4}\right| \leq|\delta|$ and we may choose to include first any $T$ 's present in $Z^{s_{2}}, Z^{s_{4}}$. Then, when the $T$ 's are exhausted we include the possible $Y$ 's present in $Z^{s_{2}}, Z^{s_{4}}$. Only when these are not available we may include $X$ 's in the $Z^{s_{2}}, Z^{s_{4}}$. At the end of this process we are left with a term $Z^{s_{3}}$ of the form $Z^{s_{3}}=T^{b_{1}} V^{b_{2}}$, where, as above, $V$ denotes either an $X$ or a $Y$ derivative, 
$b_{1}<q$ provided $q>0$. We sum up what we have done up to now in the following equality:

$$
\begin{aligned}
V^{I} T^{q} & {\left[\left(T^{p}\right)_{\left(\varphi_{i} \psi_{i}\right)^{(r)}}, \tilde{G}\right] V^{K} } \\
= & V^{I} T^{q} R_{\left[\left(T^{p}\right)_{\left(\varphi_{i} \psi_{i}\right)}(r), \tilde{G}\right], N_{i}} V^{K} \\
& +\sum V^{I} T^{q}\left(E_{r, i, \tilde{G}}^{1}+E_{r, i, \tilde{G}}^{2}\right) V^{K} \\
& +\underline{C^{N_{i}}} \sum_{\substack{\tau+\left|\alpha^{\prime}+\beta^{\prime}\right| \leq p \\
|\gamma+\delta| \leq N_{i}}} \frac{1}{\alpha ! \beta ! \gamma ! \delta !} \tilde{G}_{\left(\alpha^{\prime}, \beta^{\prime}, \tau, \delta, s_{1}\right)}^{(\gamma)} Z^{s_{2}} Z^{s_{3}} Z^{s_{4}} \\
& \circ a d_{Z}^{s_{5}}\left(B_{\alpha \beta}\left(\left(\varphi_{i} \psi_{i}\right)^{(r)}\right)_{(\gamma)}^{(\delta)}\right)
\end{aligned}
$$

where $Z^{s_{3}}=T^{b_{1}} V^{b_{2}}, b_{1}<q$ if $q>0$ and $\sum_{i=1}^{5} s_{i}=|I+K|+p+q-\left|\alpha^{\prime}+\beta^{\prime}\right|-\tau$, $\left|s_{2}\right| \leq|\gamma|,\left|s_{4}\right| \leq|\delta|$ (equalities hold unless $s_{3}=0$ ).

Before proceeding we want to bring all the $\tilde{G}_{(\cdot)}^{(\cdot)}$ to the left. Writing $\tilde{\alpha}=\alpha-\alpha^{\prime}$, $\tilde{\beta}=\beta-\beta^{\prime}$ we get

$$
\begin{aligned}
V^{I} T^{q} & {\left[\left(T^{p}\right)_{\left(\varphi_{i} \psi_{i}\right)^{(r)},}, \tilde{G}\right] V^{K} } \\
= & V^{I} T^{q} R_{\left[\left(T^{p}\right)_{\left(\varphi_{i} \psi_{i}\right)(r)}, \tilde{G}\right], N_{i}} V^{K} \\
& +\sum_{\substack{1 \leq\left|\alpha^{\prime}+\beta^{\prime}+\gamma+\delta\right|+\tau \leq p \\
I^{\prime} \leq I, q^{\prime} \leq q}}(-1)^{\left|\alpha^{\prime}\right|}\left(\begin{array}{c}
\alpha \\
\alpha^{\prime}
\end{array}\right)\left(\begin{array}{c}
\beta \\
\beta^{\prime}
\end{array}\right)\left(\begin{array}{c}
|I| \\
\left|I^{\prime}\right|
\end{array}\right)\left(\begin{array}{c}
q \\
q^{\prime}
\end{array}\right) \tilde{G}_{\left(\alpha^{\prime}, \beta^{\prime}, \tau, \delta,\left|I^{\prime}\right|, q^{\prime}\right)}^{(\gamma)} \\
& \cdot T^{|\gamma|} V^{I-I^{\prime}} T^{q-q^{\prime}} \\
& \cdot \sum_{|\tilde{\alpha}+\tilde{\beta}| \leq p-\left|\alpha^{\prime}+\beta^{\prime}\right|-\tau-|\gamma+\delta|}(-1)^{|\tilde{\alpha}| \mid}\left(\begin{array}{c}
p-\left|\alpha^{\prime}+\beta^{\prime}\right|-|\tilde{\alpha}+\tilde{\beta}| \\
\tau
\end{array}\right) \\
& \times \frac{1}{\alpha ! \beta ! \gamma ! \delta !} A_{\tilde{\alpha} \tilde{\beta}}^{p-\left|\alpha^{\prime}+\beta^{\prime}\right|-\tau-|\gamma+\delta|} T^{|\delta|} B_{\tilde{\alpha}+\alpha^{\prime}, \tilde{\beta}+\beta^{\prime}}\left(\left(\varphi_{i} \psi_{i}\right)^{(r)}\right)_{(\gamma)}^{(\delta)} V^{K} \\
& +\sum_{p-\tau-|\gamma+\delta| \leq|\alpha+\beta| \leq p-\tau} \sum_{\substack{I^{\prime} \leq I \\
q^{\prime} \leq q}}(-1)^{|\alpha|}\left(\begin{array}{c}
\alpha \\
\alpha^{\prime}
\end{array}\right)\left(\begin{array}{c}
\beta \\
\beta^{\prime}
\end{array}\right)\left(\begin{array}{c}
p-|\alpha+\beta| \\
\tau
\end{array}\right) \\
& \times\left(\begin{array}{c}
|I| \alpha^{\prime}+\tilde{\beta}^{\prime}, \beta^{\prime} \leq \beta \\
\left|I^{\prime}\right|
\end{array}\right)\left(\begin{array}{c}
q \\
q^{\prime}
\end{array}\right) \frac{1}{\alpha ! \beta ! \gamma \mid+\tau \leq p} \tilde{G}_{\left(\alpha^{\prime}, \beta^{\prime}, \tau, \delta,\left|I^{\prime}\right|, q^{\prime}\right)}^{(\gamma)} \\
& \times Z^{|\gamma|} T^{q-q^{\prime}} V^{I-I^{\prime}+p-\left|\alpha^{\prime}+\beta^{\prime}+\gamma+\delta\right|-\tau} Z^{|\delta|} B_{\alpha \beta}\left(\left(\varphi_{i} \psi_{i}\right)^{(r)}\right)_{(\gamma)}^{(\delta)} V^{K}
\end{aligned}
$$




$$
\begin{aligned}
& +\sum_{\substack{N_{i} \\
\left|s_{1}\right|+\left|b_{1}\right|+\left|b_{2}\right|+|\gamma+\delta|=|I+K|+p+q-\left|\alpha^{\prime}+\beta^{\prime}\right|-\tau \\
\alpha^{\prime} \leq \alpha, \beta^{\prime} \leq \beta,\left|\alpha^{\prime}+\beta^{\prime}\right|+\tau \leq p \\
b_{1}<q}} \frac{1}{\alpha ! \beta ! \gamma ! \delta !} \tilde{G}_{\substack{q>0 \\
\left(\alpha^{\prime}, \beta^{\prime}, \tau, \delta, s_{1}\right)}}^{(\gamma)} \\
& \times Z^{|\gamma|} T^{b_{1}} V^{b_{2}} B_{\alpha \beta}\left(\left(\varphi_{i} \psi_{i}\right)^{(r)}\right)_{(\gamma)}^{(\delta)} \\
& +\sum^{N_{i}} \sum_{\substack{\left|s_{2}+s_{4}\right| \leq|\gamma+\delta| \leq N_{i} \\
\left|s_{1}+s_{2}+s_{4}\right| \leq|+K|+p+q-\left|\alpha^{\prime}+\beta^{\prime}\right|-\tau}} \frac{1}{\alpha ! \beta ! \gamma ! \delta !} \tilde{G}_{\left(\alpha^{\prime}, \beta^{\prime}, \tau, \delta, s_{1}\right)}^{(\gamma)} \\
& \cdot Z^{s_{2}+s_{4}} B_{\alpha \beta}\left(\left(\varphi_{i} \psi_{i}\right)^{(r)}\right)_{(\gamma)}^{(\delta)} \text {. }
\end{aligned}
$$

The second term on the right needs rewriting if it is to be brought into a form where the $\Sigma A_{\tilde{\alpha} \tilde{\beta}} B_{\alpha^{\prime}+\tilde{\alpha}, \beta^{\prime}+\tilde{\beta}}\left(\left(\varphi_{i} \psi_{i}\right)^{(r)}\right) \frac{1}{\tilde{\alpha} ! \tilde{\beta} !}\left(\begin{array}{c}p-\left|\alpha^{\prime}+\tilde{\alpha}+\beta^{\prime}+\tilde{\beta}\right| \\ \tau\end{array}\right)$ have the right balance, as in (4.2.1). First we write

$$
\begin{aligned}
& \left(\begin{array}{c}
p-\left|\alpha^{\prime}+\tilde{\alpha}+\beta^{\prime}+\tilde{\beta}\right| \\
\tau
\end{array}\right) \\
& =\sum_{\ell \leq|\tilde{\alpha}+\tilde{\beta}|, \tau}(-1)^{\ell}\left(\begin{array}{c}
p-\left|\alpha^{\prime}+\beta^{\prime}\right|-\ell \\
\tau-\ell
\end{array}\right)\left(\begin{array}{c}
|\tilde{\alpha}+\tilde{\beta}| \\
\ell
\end{array}\right) \\
& =\sum_{\ell \leq|\tilde{\alpha}+\tilde{\beta}|, \tau}(-1)^{\ell}\left(\begin{array}{c}
p-\left|\alpha^{\prime}+\beta^{\prime}\right|-\ell \\
\tau-\ell
\end{array}\right) \sum_{\substack{\alpha^{\prime \prime}+\beta^{\prime \prime} \mid \leq \ell \\
\alpha^{\prime \prime} \leq \tilde{\alpha}, \beta^{\prime \prime} \leq \tilde{\beta}}}\left(\begin{array}{c}
\tilde{\alpha} \\
\alpha^{\prime \prime}
\end{array}\right)\left(\begin{array}{c}
\tilde{\beta} \\
\beta^{\prime \prime}
\end{array}\right) .
\end{aligned}
$$

Proposition 5.3.1. Any expression of the form

$$
V^{\delta} a d_{V}^{\tau_{1}}\left(a d_{V}^{\tau_{2}} B_{\alpha ! \beta !}\left(a d_{V}^{\tau_{3}}(\varphi \psi)^{(r)}\right)\right)_{(\gamma)}^{(\delta)} V^{K}
$$

may be written as a sum of $C^{\left|\rho_{1}\right|}$ terms, $\left|\rho_{1}\right|=\left|\delta+\Sigma \tau_{1}+\gamma\right|+|K|$, each of the form

$$
x^{\tilde{\rho}_{1}} B_{\tilde{a}, \tilde{b}}\left((\varphi \psi)^{\left(\left|\rho_{1}\right|+r\right)}\right),
$$

where $\left|\tilde{\rho}_{1}\right| \leq\left|\rho_{1}\right|=\left|\delta+\Sigma \tau_{1}+\gamma\right|+|K|$.

Remark 5.3.1. This is where the precise form of the vector fields introduced in Definition 4.3.2 is needed. In particular, any $x$ - or $\xi$-derivative of a symbol can be thought of as the action of a $Z_{\sigma}$ field on the symbol itself. We point out explicitly that the $W_{\sigma}$ and $\Xi_{\sigma}$ derivatives may be commuted with $B_{\alpha, \beta}$ and go directly onto their argument, see e.g. (4.3.16).

Proposition 5.3.1 is very easy to prove.

Proof. This is a repeated use of Lemma A.3. An expression of the form $V^{\delta} \circ f(x, D)$ may be written as $C^{|\delta|}$ terms of the type $x^{\delta^{\prime}} W^{\delta} \circ f(x, D), \delta^{\prime} \leq \delta$. Using the definition of the vector fields in Definition 4.3.2 and Lemma A.3 we prove the result.

Using the argument of Proposition 5.3.1 we may write

$$
\begin{aligned}
T^{|\delta|} & \left(B_{\tilde{\alpha}+\alpha^{\prime}, \tilde{\beta}+\beta^{\prime}}\left(\left(\varphi_{i} \psi_{i}\right)^{(r)}\right)_{(\gamma)}^{(\delta)}\right) \\
= & C^{\left|\gamma+\delta+\alpha^{\prime}+\delta^{\prime}\right|} x^{\rho^{\prime}} B_{\tilde{\alpha} \tilde{\beta}}\left(\left(\varphi_{i} \psi_{i}\right)^{\left(r+\left|\gamma+\delta+\alpha^{\prime}+\beta^{\prime}\right|\right)}\right)_{(\gamma)}^{(\delta)}
\end{aligned}
$$

where $\left|\rho^{\prime}\right| \leq\left|\gamma+\delta+\alpha^{\prime}+\beta^{\prime}\right|$. 
Let us focus our attention on the second term of (5.3.19) and take into account (5.3.20): the coefficient $\left(\begin{array}{c}\tilde{\alpha} \\ \alpha^{\prime \prime}\end{array}\right)\left(\begin{array}{c}\tilde{\beta} \\ \beta^{\prime \prime}\end{array}\right) \frac{1}{\tilde{\alpha} ! \tilde{\beta} !}=\frac{1}{\alpha^{\prime \prime} ! \underline{!} ! \beta^{\prime \prime} ! \underline{\beta} !}$, where $\underline{\alpha}=\tilde{\alpha}-\alpha^{\prime \prime}, \underline{\beta}=\tilde{\beta}-\beta^{\prime \prime}$, makes it appear that $\underline{\alpha}, \underline{\beta}$ should be the running indices, not $\tilde{\alpha}, \tilde{\beta}$. Well,

$$
A_{\tilde{\alpha} \tilde{\beta}}^{\left(p_{1}\right)}=X^{\prime \prime \alpha^{\prime \prime}} X^{\prime \prime} \underline{\alpha} X^{\prime} \underline{\beta} X^{\prime \beta^{\prime \prime}} T^{p_{1}-\left|\alpha^{\prime \prime}+\beta^{\prime \prime}\right|-|\underline{\alpha}-\underline{\beta}|},
$$

but now the vector fields $X^{\prime \beta^{\prime \prime}}$ are in the wrong position. To manage this type of term, where, if we maintain the balance needed for iteration, then the vector fields are in unadmissible locations, we have found it helpful to write

$$
\begin{aligned}
& \left(T^{p_{1}}\right)_{X^{\prime \sigma}, x^{\prime \rho}, a d_{X^{\prime}}^{\tau},\left((\tilde{\varphi} \tilde{\psi})^{(r)}\right)} \\
& =\sum_{\left|\alpha_{1}+\beta_{1}\right| \leq p_{1}} \frac{(-1)^{\left|\alpha_{1}\right|}}{\alpha_{1} ! \beta_{1} !} A_{\alpha_{1} \beta_{1}}^{\left(p_{1}\right)} X^{\prime \sigma} x^{\rho \rho} a d_{X^{\prime}}^{\tau} B_{\alpha_{1} \beta_{1}}\left((\tilde{\varphi} \tilde{\psi})^{(r)}\right) .
\end{aligned}
$$

Thus the second term on the right of (5.3.19) becomes

$$
\begin{aligned}
& \sum_{\substack{1 \leq\left|\alpha^{\prime}+\beta^{\prime}+\gamma+\delta\right|+\tau \leq p \\
I^{\prime} \leq I, q^{\prime} \leq q}}(-1)^{\left|\alpha^{\prime}\right|}\left(\begin{array}{c}
\alpha \\
\alpha^{\prime}
\end{array}\right)\left(\begin{array}{c}
\beta \\
\beta^{\prime}
\end{array}\right)\left(\begin{array}{c}
|I| \\
\left|I^{\prime}\right|
\end{array}\right)\left(\begin{array}{c}
q \\
q^{\prime}
\end{array}\right) \tilde{G}_{\left(\alpha^{\prime}, \beta^{\prime}, \tau, \delta, I^{\prime}, q^{\prime}\right)}^{(\gamma)} \\
& \times T^{|\gamma|} V^{I-I^{\prime}} T^{q-q^{\prime}} \sum_{\substack{\left|\alpha^{\prime \prime}+\beta^{\prime \prime}\right|=\ell \leq|\tilde{\alpha}+\tilde{\beta}|, \tau \\
\alpha^{\prime \prime} \leq \tilde{\alpha}, \beta^{\prime \prime} \leq \tilde{\beta}}}(-1)^{\ell}\left(\begin{array}{c}
p-\left|\alpha^{\prime}+\beta^{\prime}\right|-\ell \\
\tau-\ell
\end{array}\right) \\
& \times \frac{(-1)^{\left|\alpha^{\prime \prime}\right|}}{\alpha^{\prime \prime} ! \beta^{\prime \prime !} !} \frac{C^{\left|\gamma+\delta+\alpha^{\prime}+\beta^{\prime}\right|}}{X^{\prime \prime \alpha^{\prime \prime}}} \\
& \times\left(T^{p-\left|\alpha^{\prime}+\beta^{\prime}\right|-\left|\alpha^{\prime \prime}+\beta^{\prime \prime}\right|-\tau-|\gamma+\delta|}\right)_{X^{\prime \beta^{\prime \prime}}, x^{\prime \rho^{\prime}}, a d_{X^{\prime}}^{\alpha^{\prime \prime}},\left(\left(\varphi_{i} \psi_{i}\right)^{\left(r+\left|\gamma+\delta+\alpha^{\prime}+\beta^{\prime}+\beta^{\prime \prime}\right|\right)}\right)} V^{K},
\end{aligned}
$$

where $\left|\rho^{\prime}\right| \leq\left|\gamma+\delta+\alpha^{\prime}+\beta^{\prime}\right|$.

First of all let us now deal with the $X^{\prime \beta^{\prime \prime}}$ :

$$
\begin{aligned}
& \left(T^{p_{1}}\right)_{X^{\prime \beta^{\prime \prime}}, x^{\prime \rho^{\prime}}, a d_{X}^{\alpha^{\prime \prime}},\left((\tilde{\varphi} \tilde{\psi})^{(r)}\right)}=C^{\left|\beta^{\prime \prime}\right|}\left(T^{p_{1}}\right)_{X_{1}^{\prime \beta_{1}^{\prime \prime}}\left(x^{\prime \rho^{\prime}}\right), a d_{X^{\prime}}^{\alpha^{\prime \prime}+\beta_{2}^{\prime \prime}},\left((\tilde{\varphi} \tilde{\psi})^{(r)}\right)} X^{\prime \beta_{3}^{\prime \prime}}
\end{aligned}
$$

where $\sum \beta_{i}^{\prime \prime}=\beta^{\prime \prime}$. Now

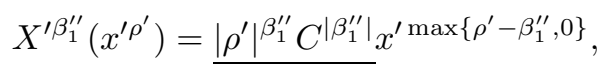

and, as above

$$
a d_{X^{\prime}}^{\alpha^{\prime \prime}+\beta_{2}^{\prime \prime}}\left(B_{\underline{\alpha} \underline{\beta}}\left((\tilde{\varphi} \tilde{\psi})^{(r)}\right)\right)=C^{\left|\alpha^{\prime \prime}+\beta_{2}^{\prime \prime}\right|} x^{\prime \rho_{2}} B_{\underline{\alpha} \underline{\beta}}\left((\tilde{\varphi} \tilde{\psi})^{(r+|\vartheta|)}\right),
$$

where $\left|\rho_{2}\right| \leq\left|\alpha^{\prime \prime}+\beta_{2}^{\prime \prime}\right|,|\vartheta| \leq\left|\alpha^{\prime \prime}+\beta_{2}^{\prime \prime}\right|$. Hence

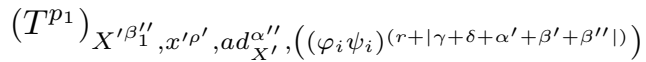

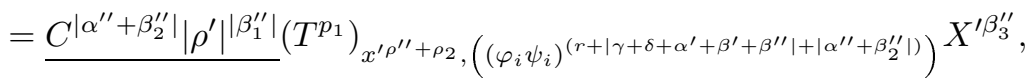

where $\sum \beta_{i}^{\prime \prime}=\beta^{\prime \prime}$. 
From the definition we have

$$
\begin{aligned}
\left(T^{p_{1}}\right)_{X_{j}^{\prime \prime},(\varphi \psi)^{(r)}} & =x_{j}^{\prime \prime}\left(T^{p_{1}}\right)_{(\varphi \psi)^{(r)}}+\left(T^{p_{1}-1}\right)_{a d_{X^{\prime}},(\varphi \psi)^{(r)}} \\
& =x_{j}^{\prime \prime}\left(T^{p_{1}}\right)_{(\varphi \psi)^{(r)}}+2\left(T^{p_{1}-1}\right)_{X,(\varphi \psi)^{(r+1)}} \\
& =\cdots \\
& =\sum_{k=0}^{p_{1}} \underline{C^{k}} x_{j}^{\prime \prime}\left(T^{p_{1}-k}\right)_{(\varphi \psi)^{(r+k)}}
\end{aligned}
$$

and

$$
\left(T^{p_{1}}\right)_{X_{j}^{\prime},(\varphi \psi)^{(r)}}=x_{j}^{\prime}\left(T^{p_{1}}\right)_{(\varphi \psi)^{(r)}}+\left(T^{p_{1}-1}\right)_{(\varphi \psi)^{(r+1)}}
$$

so that, for several $X$ 's, with $\sum k_{i}=k$, we obtain

$$
\left(T^{p_{1}}\right)_{X^{\varepsilon},(\varphi \psi)^{(r)}}
$$

$$
\begin{aligned}
& =\sum_{k_{1}=0}^{p_{1}} \sum_{k_{2}=0}^{p_{1}-k_{1}} \cdots \sum_{k_{|\varepsilon|=0}}^{p_{1}-k_{1}-\cdots-k_{|\varepsilon|-1}} \underline{C^{k_{1}+k_{2}+\cdots+k_{|\varepsilon|}}} x^{\varepsilon^{\prime}}\left(T^{p_{1}-k}\right)_{(\varphi \psi)^{(r+k)}} \\
& =\sum_{k=0}^{p_{1}} C^{k}\left(\begin{array}{c}
|\varepsilon|+k-1 \\
k
\end{array}\right) x^{\varepsilon^{\prime}}\left(T^{p_{1}-k}\right)_{(\varphi \psi)^{(r+k)}},
\end{aligned}
$$

where $\left|\varepsilon^{\prime}\right| \leq|\varepsilon|$. Furthermore, since

$$
\begin{aligned}
& \left(\begin{array}{c}
p-\left|\alpha^{\prime}+\beta^{\prime}\right|-\left|\alpha^{\prime \prime}+\beta^{\prime \prime}\right| \\
\tau-\left|\alpha^{\prime \prime}+\beta^{\prime \prime}\right|
\end{array}\right) \frac{1}{\alpha^{\prime \prime} ! \beta^{\prime \prime} !} \\
& \leq \frac{C}{\tau !}\left(\begin{array}{c}
\tau \\
\left|\alpha^{\prime \prime}+\beta^{\prime \prime}\right|
\end{array}\right) \frac{\left(p-\left|\alpha^{\prime}+\beta^{\prime}\right|-\left|\alpha^{\prime \prime}+\beta^{\prime \prime}\right|\right) !}{\left(p-\left|\alpha^{\prime}+\beta^{\prime}\right|-\tau\right) !} \\
& \leq C^{\tau} \frac{N_{i}^{\tau-\left|\alpha^{\prime \prime}+\beta^{\prime \prime}\right|}}{\tau !},
\end{aligned}
$$

we may estimate (5.3.24) applied to $u$ in $L^{2}$-norms by

$$
\begin{aligned}
& \sup _{\substack{1 \leq\left|\alpha^{\prime}+\beta^{\prime}+\gamma+\delta\right|+\tau \leq p \\
I^{\prime} \leq I, q^{\prime} \leq q,\left|\alpha^{\prime \prime}+\beta^{\prime \prime}\right| \leq \tau \\
\left|\rho^{\prime}, \leq\right| \alpha^{\prime}+\beta^{\prime}+\gamma+\gamma| \\
1 \leq p-| \alpha^{\prime}+\beta^{\prime}+\alpha^{\prime \prime}+\beta^{\prime \prime}+\gamma+\delta \mid-\tau \\
\alpha^{\prime \prime} \leq \alpha^{\prime}, \sum \beta_{i}^{\prime \prime}=\beta^{\prime \prime} \leq \beta^{\prime}}} \underline{C^{\left|\gamma+\delta+\alpha^{\prime}+\beta^{\prime}\right|+\tau+k+q^{\prime}+\left|I^{\prime}\right|}} \\
& \quad \times \frac{N_{i}^{d_{3}}}{N_{i}^{d_{2}\left(q^{\prime}+\tau+\left|\alpha^{\prime}+\beta^{\prime}+\gamma+\delta\right|+|I|\right) !}} \\
& \quad \times \| \tilde{G}_{\left(\alpha^{\prime}, \beta^{\prime}, \tau,\left|I^{\prime}\right|, q^{\prime}\right)}^{(\gamma)} T^{|\gamma|} V^{I-I^{\prime}+\alpha^{\prime \prime}} T^{q-q^{\prime}} x^{\rho^{\prime}+\alpha^{\prime \prime}+\beta^{\prime \prime}} \\
& . \quad\left(T^{p-\left|\alpha^{\prime}+\beta^{\prime}+\alpha^{\prime \prime}+\beta^{\prime \prime}+\gamma+\delta\right|-\tau-k}\right)_{\left(\varphi_{i} \psi_{i}\right)^{\left(d_{2}+r\right)}} V^{\beta_{3}^{\prime \prime}+K} u \|,
\end{aligned}
$$

where $d_{3}=\tau+k+\left|\gamma+\delta+\alpha^{\prime}+\beta^{\prime}+\alpha^{\prime \prime}+\beta^{\prime \prime}\right|+\left|I^{\prime}\right|+q^{\prime}-\left|\alpha^{\prime \prime}+\beta_{3}^{\prime \prime}\right|$ and $d_{2}=$ $\left|\gamma+\delta+\alpha^{\prime}+\beta^{\prime}+\alpha^{\prime \prime}+\beta^{\prime \prime}+\beta_{2}^{\prime \prime}\right|$. We have written out $d_{3}, d_{2}$ since $d_{2}$ is the number of new derivatives on $\left(\varphi_{i} \psi_{i}\right)$ and $d_{3}$ is the net loss of free derivatives. We point out that $d_{3} \geq 1$. Bringing the powers of $x$ to the left of $V^{I-I^{\prime}+\alpha^{\prime \prime}} T^{q-q^{\prime}}$ will not alter the form of (5.3.30) substantially, it merely adds to the supremum the condition $\left|\rho^{\prime \prime}\right| \leq\left|I-I^{\prime}+\alpha^{\prime \prime}\right|,\left|\rho^{\prime}+\alpha^{\prime \prime}+\beta^{\prime \prime}\right|$, replaces $V^{I-I^{\prime}+\alpha^{\prime \prime}}$ by $V^{I-I^{\prime}+\alpha^{\prime \prime}-\rho^{\prime \prime}}$, moves every 
$x$ to the left of $V^{I-I^{\prime}+\alpha^{\prime \prime}}$ and adds a factor $C^{\left|\rho^{\prime}+\alpha^{\prime \prime}+\beta^{\prime \prime}\right|} N_{i}^{\rho^{\prime \prime}}$ in front. Thus (5.3.30) is bounded by

$$
\sup _{\substack{\tilde{I}, \tilde{K}, \tilde{q} \leq q, \tilde{p} \leq p \\ \rho / 2, s / 2,\left|d_{1}+\gamma\right| \leq \Delta+\Delta+\Delta q+\Delta K+\Delta p \\ \Delta I+\Delta q+\Delta K+\Delta p \geq 1, \Delta q \geq 0}} \frac{\left(C N_{i}\right)^{\Delta I+\Delta q+\Delta K+\Delta p}}{N_{i}^{s}\left|\gamma+d_{1}\right| !}
$$

where $\Delta I=|I|-|\tilde{I}|, \Delta p=p-\tilde{p}, \Delta q=q-\tilde{q}, \Delta K=|K|-|\tilde{K}|$.

Remark 5.3.2. From now on we shall occasionally include a numerical multiple by $N_{i}$ as a derivative of a localizing function when this will be enough for our purposes. Thus $(\varphi \psi)^{(a+b)}$ could refer to $N^{a}(\varphi \psi)^{(b)}$. Moreover we shall restrict ourselves to the analytic type of estimates, since this will be enough for the propagation of the regularity theorem.

Using the fact that

$$
\begin{gathered}
\left(\begin{array}{c}
p-|\alpha+\beta| \\
\tau
\end{array}\right) \leq \frac{N_{i}^{\tau}}{\tau !} \\
\left(\left|\alpha^{\prime}+\beta^{\prime}+\gamma+\delta\right|+\left|I^{\prime}\right|+q^{\prime}\right) ! \leq C^{\left|\alpha^{\prime}+\beta^{\prime}+\gamma+\delta\right|+\left|I^{\prime}\right|+q^{\prime}} \alpha^{\prime} ! \beta^{\prime} ! \gamma ! \delta !\left|I^{\prime}\right| ! q^{\prime} !
\end{gathered}
$$

and applying Proposition 5.3.3, the third term in (5.3.19) is bounded by

$$
\begin{gathered}
\underline{C^{N_{i}}} \sup _{\substack{|\tilde{I}|+\tilde{q}<|I|+q+p \\
s<N_{i},\left|d_{i}+\gamma\right| \leq N_{i}, \Delta q \geq 0}} \frac{N_{i}^{\Delta I+\Delta q+p}}{N_{i}^{s}\left|d_{1}+\gamma\right| !} \\
\times\left\|\tilde{G}_{\left(d_{1}\right)}^{(\gamma)} Z^{|\gamma|} T^{\tilde{q}} V^{\tilde{I}}\left(\varphi_{i} \psi_{i}\right)^{s+r}\right\|,
\end{gathered}
$$

again with $\Delta I=|I|-|\tilde{I}|, \Delta p=p-\tilde{p}, \Delta q=q-\tilde{q}$, all non negative in this case.

The last two terms in (5.3.19) are similarly treated. For the next to last we get the bound

$$
\begin{gathered}
\underline{C^{N_{i}}} \sup _{\substack{|\tilde{I}| \leq|I|, \tilde{q} \leq q+p \\
s<N_{i},\left|d_{i}+\gamma\right| \leq N_{i}}} \frac{N_{i}^{\Delta I+|K|+\Delta q+p}}{N_{i}^{s}\left|d_{1}+\gamma\right| !} \\
\times \quad\left\|\tilde{G}_{\left(d_{1}\right)}^{(\gamma)} Z^{|\gamma|} T^{\tilde{q}} V^{\tilde{I}}\left(\varphi_{i} \psi_{i}\right)^{s+r}\right\| .
\end{gathered}
$$

As for the last term, using also Remark 5.3.2, we have the bound

$$
\begin{aligned}
& \underline{C}^{N_{i}} \sup _{\substack{\left|s_{2}\right| \leq|\gamma|,\left|s_{4}\right| \leq|\delta| \\
\left|d_{1}+\gamma\right|,|\gamma+\delta| \leq N_{i} \\
|s| \leq N_{i}}} \frac{N_{i}^{|\gamma|-\left|s_{2}\right|} N_{i}^{K}}{N_{i}^{s} N_{i}^{|\gamma|-\left|s_{2}\right|+|\delta|-\left|s_{4}\right|}} \frac{1}{\alpha ! \beta ! \gamma ! \delta !} \\
& \text { - }\left\|\tilde{G}_{\left(d_{1}\right)}^{(\gamma)} Z^{s_{2}}\left(\varphi_{i} \psi_{i}\right)^{(r+s)} V^{K} u\right\|
\end{aligned}
$$

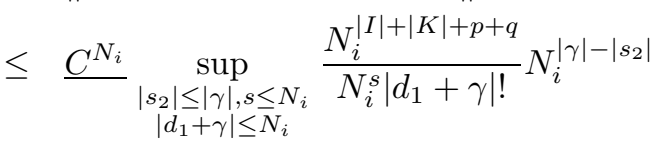

$$
\begin{aligned}
& \text {. }\left\|\tilde{G}_{\left(d_{1}\right)}^{(\gamma)} Z^{s_{2}}\left(\varphi_{i} \psi_{i}\right)^{(r+s)} u\right\|,
\end{aligned}
$$

where the factor $N_{i}^{|\gamma|-\left|s_{2}\right|}$ will later go with $\tilde{G}_{\left(d_{1}\right)}^{(\gamma)} Z^{s_{2}}$. 
To bring $\tilde{G}_{\left(d_{1}\right)}^{(\gamma)} Z^{s^{\prime}},\left|s^{\prime}\right| \leq|\gamma|$, out of the $L^{2}$-norm we must recall that the symbol of $\tilde{G}$ has been cut-off to be zero for $|\xi| \leq N_{i}$ :

$$
\begin{aligned}
\sigma\left(\tilde{G}_{\left(d_{1}\right)}^{(\gamma)}\right)(x, \xi) & =\partial_{x}^{d_{1}} \partial_{\xi}^{\gamma}\left(\Phi(x) g(x, \xi) \Psi_{i}^{\prime}(\xi)\right) \\
& =g_{\left(d_{1}\right)}^{(\gamma)}(x, \xi) \Psi_{i}^{\prime}(\xi)+\sum_{1 \leq\left|\gamma_{1}\right| \leq|\gamma|} g_{\left(d_{1}\right)}^{\left(\gamma-\gamma_{1}\right)}(x, \xi) \Psi_{i}^{\prime\left(\gamma_{1}\right)}(\xi),
\end{aligned}
$$

when $x \in \Omega$, since $\Phi(x) \equiv 1$ in a neighborhood of $\bar{\Omega}$.

Since $Z^{s^{\prime}}$ is at most $C^{|\gamma|}\left(\begin{array}{c}\left|s^{\prime}\right| \\ \left|\gamma^{\prime}\right|\end{array}\right)\left|\gamma^{\prime}\right| ! \leq \tilde{C}^{|\gamma|}\left|\gamma^{\prime}\right|$ ! terms of the form $D^{s^{\prime}-\gamma^{\prime}} x^{s^{\prime}-\gamma^{\prime}}$, for some $\gamma^{\prime} \leq s^{\prime}$, we may write

$$
\begin{aligned}
& \left\|\operatorname{Op}\left(g_{\left(d_{1}\right)}^{(\gamma)}(x, \xi) \Psi_{i}^{\prime}(\xi)\right) D^{s^{\prime}-\gamma^{\prime}} w\right\| \\
& \leq \sup _{\substack{|\rho| \leq n+1 \\
|\xi|}}\left|g_{\left(d_{1}+\rho\right)}^{(\gamma)}(x, \xi) \xi^{s^{\prime}-\gamma^{\prime}} \Psi_{i}^{\prime}(\xi)\right|\|w\| \\
& \leq \sup _{\substack{|\rho| \leq n+1 \\
|\xi|}}\left[C^{\left|d_{1}+\rho+\gamma\right|}\left(d_{1}+\rho\right) !|\gamma| ! \frac{|\xi|^{\left|s^{\prime}-\gamma\right|} \Psi_{i}^{\prime}(\xi)}{(1+|\xi|)^{|\gamma|}}\right]\|w\| \\
& \leq C C^{\left|d_{1}+\gamma\right|} \frac{d_{1} !\left(\gamma-s^{\prime}\right) ! s^{\prime} !}{N_{i}^{\left|\gamma-s^{\prime}\right|+\left|\gamma^{\prime}\right|}},
\end{aligned}
$$

so that

$$
\left\|\operatorname{Op}\left(g_{\left(d_{1}\right)}^{(\gamma)}(x, \xi) \Psi_{i}^{\prime}(\xi)\right)\right\|_{L^{2} \rightarrow L^{2}} \leq C C^{\left|d_{1}+\gamma\right|}\left(d_{1}+s^{\prime}\right) !
$$

if $w$ has support in a compact set (so $|x|^{\left|s^{\prime}-\gamma^{\prime}\right|} \leq C^{\left|s^{\prime}-\gamma^{\prime}\right|} \leq C^{|\gamma|}$ ) and $\xi$ may be taken to lie in $\tilde{\Gamma}_{i}$, which we will be able to do since $w$ will contain $\varphi_{i} \psi_{i}$. Again, for sake of simplicity we are assuming that $g$ (and thus $P$ ) has "analytic coefficients".

As for the case $\left|\gamma_{1}\right| \geq 1$ (i.e. when some derivatives land on $\Psi_{i}^{\prime}(\xi)$ ) the expression $g_{\left(d_{1}\right)}^{\left(\gamma-\gamma_{1}\right)}(x, \xi) \Psi_{i}^{\prime\left(\gamma_{1}\right)}(\xi) \xi^{s^{\prime}-\gamma^{\prime}}$ has $\xi$-support in $\left\{N_{i} \leq|\xi| \leq 2 N_{i}\right\}$ and

$$
\begin{aligned}
& \sup _{\substack{|\rho| \leq n+1 \\
N_{i} \leq|\xi| \leq 2 N_{i}}}\left|\partial_{\xi}^{\rho}\left(g_{\left(d_{1}\right)}^{\left(\gamma-\gamma_{1}\right)}(x, \xi) \Psi_{i}^{\prime\left(\gamma_{1}\right)}(\xi) \xi^{s^{\prime}-\gamma^{\prime}}\right)\right| \\
& \leq \sup _{\substack{|\rho| \leq n+1 \\
|\xi|}} C^{\left|d_{1}+\gamma\right|+n+1} d_{1} !\left(\left|\gamma-\gamma_{1}\right|+|\rho|\right) ! \frac{|\xi|^{\left|s^{\prime}-\gamma^{\prime}\right|}}{(1+|\xi|)^{\left|\gamma-\gamma_{1}\right|}} \chi_{\left\{N_{i} \leq|\xi| \leq 2 N_{i}\right\}} \\
& \leq \quad C C^{\left|d_{1}+\gamma\right|} \sup _{\substack{|\rho| \leq n+1, \gamma^{\prime} \leq s^{\prime} \\
N_{i} \leq|\xi| \leq 2 N_{i}}} \frac{d_{1} !\left(\left|\gamma-\gamma_{1}\right|+|\rho|\right) !}{(1+|\xi|)^{\left|\gamma-s^{\prime}+\gamma^{\prime}-\gamma_{1}\right|}} \\
& \leq C C^{\left|d_{1}+\gamma\right|} \frac{d_{1} !}{N_{i}^{\left|\gamma^{\prime}-s^{\prime}\right|}},
\end{aligned}
$$

so that for $|\gamma| \geq 1$, due to the fact that $\frac{|\gamma| \gamma^{\left|\gamma^{\prime}\right|}}{N_{i}^{\left|\gamma^{\prime}\right|}} \leq 1$,

$$
\begin{aligned}
& \left\|\operatorname{Op}\left(g_{\left(d_{1}\right)}^{\left(\gamma-\gamma_{1}\right)}(x, \xi) \Psi_{i}^{\prime\left(\gamma_{1}\right)}(\xi)\right) Z^{s_{1}} w\right\| \\
& \quad \leq C C^{\left|d_{1}+\gamma\right|} d_{1} ! N_{i}^{\left|s^{\prime}\right|} \sup _{\gamma^{\prime} \leq s^{\prime}}\left\|\operatorname{Op}\left(\chi_{\left\{N_{i} \leq|\xi| \leq 2 N_{i}\right\}}\right) x^{s^{\prime}-\gamma^{\prime}} w\right\| .
\end{aligned}
$$


Since in our case $w=T^{\tilde{q}} V^{\tilde{I}}\left(\varphi_{i} \psi_{i}\right)^{(r+s)} V^{\tilde{K}} u$, so that the conic support of $w$ lies in $\tilde{\Gamma}_{i}$, we write $x^{s^{\prime}-\gamma^{\prime}} T^{\tilde{q}} V^{\tilde{I}}\left(\varphi_{i} \psi_{i}\right)^{(r+s)} V^{\tilde{K}}$ with all the $V$ 's at the left:

$$
x^{s^{\prime}-\gamma^{\prime}} T^{\tilde{q}} V^{\tilde{I}}\left(\varphi_{i} \psi_{i}\right)^{(r+s)} V^{\tilde{K}}=\underline{C^{\tilde{K}}} x^{s^{\prime}-\gamma^{\prime}} T^{\tilde{q}} V^{\tilde{I}+K^{\prime}}\left(\varphi_{i} \psi_{i}\right)^{\left(r+s+\left|\tilde{K}-K^{\prime}\right|\right)},
$$

for some $K^{\prime} \leq \tilde{K}$, and each of these terms is bounded by

$$
N_{i}^{\rho^{\prime}} D^{\tilde{q}+\tilde{I}+K^{\prime}-\rho^{\prime}} x^{s^{\prime}-\gamma^{\prime}+\left|\tilde{I}+K^{\prime}\right|-\rho^{\prime}}\left(\varphi_{i} \psi_{i}\right)^{\left(r+s+\left|\tilde{K}-K^{\prime}\right|\right)},
$$

with $\rho^{\prime} \leq \min \left\{\tilde{q}+\tilde{I}+K^{\prime}, s^{\prime}-\gamma^{\prime}+\tilde{I}+K^{\prime}\right\}$. Similarly, using the definition of $\left(T^{p}\right)_{\left(\varphi_{i} \psi_{i}\right)(r+s)}, x^{\rho^{\prime}} T^{\tilde{q}} V^{\tilde{I}}\left(T^{p}\right)_{\left(\varphi_{i} \psi_{i}\right)^{(r+s)}} V^{\tilde{K}}$ is at most $C^{N_{i}}$ terms, each less then $N_{i}^{\rho^{\prime}}$ terms of the form

$$
\frac{1}{\alpha ! \beta !} D^{\tilde{q}+\tilde{I}+\tilde{p}+K^{\prime}-\rho^{\prime}} x^{\rho+\tilde{p}+\tilde{K}+\tilde{I}-\rho^{\prime}}\left(\varphi_{i} \psi_{i}\right)^{\left(|\alpha+\beta|+r+s+\left|\tilde{K}-K^{\prime}\right|\right)}
$$

for some $\alpha, \beta, \rho^{\prime}, K^{\prime}$ with $|\alpha+\beta| \leq \tilde{p}, K^{\prime} \leq \tilde{K}$ and

$$
|\rho| \leq \min \left\{\tilde{q}+|\tilde{I}|+\tilde{p}+\left|K^{\prime}\right|,|\rho|+|\tilde{I}|+\tilde{p}+|\tilde{K}|\right\} .
$$

Thus

$$
\begin{aligned}
& \left\|\chi_{\left\{N_{i} \leq|\xi| \leq 2 N_{i}\right\}} x^{s^{\prime}-\gamma^{\prime}} T^{\tilde{q}} V^{\tilde{I}}\left(\varphi_{i} \psi_{i}\right)^{(r+s)} V^{\tilde{K}} u\right\| \\
& \quad \leq \sup _{K^{\prime} \leq \tilde{K}} N_{i}^{\tilde{q}+\left|\tilde{I}+K^{\prime}\right|}\left\|\left(\varphi_{i} \psi_{i}\right)^{\left(r+s+\left|\tilde{K}-K^{\prime}\right|\right)} u\right\|
\end{aligned}
$$

and

$$
\begin{aligned}
& \left\|\chi_{\left\{N_{i} \leq|\xi| \leq 2 N_{i}\right\}} x^{\rho} T^{\tilde{q}} V^{\tilde{I}}\left(T^{p}\right)_{\left(\varphi_{i} \psi_{i}\right)^{(r+s)}} V^{\tilde{K}} u\right\| \\
& \leq C^{N_{i}} \sup _{\substack{\alpha \beta \\
K^{\prime} \leq \tilde{K}}} N_{i}^{\tilde{q}+\tilde{p}+\left|\tilde{I}+K^{\prime}\right|-|\alpha+\beta|}\left\|\left(\varphi_{i} \psi_{i}\right)^{\left(r+s+\left|\tilde{K}-K^{\prime}\right|+|\alpha+\beta|\right)} u\right\| .
\end{aligned}
$$

Let us now use (5.3.35), (5.3.37) in (5.3.31)- (5.3.32). Thus (5.3.31) can be bounded by

$$
\begin{aligned}
\frac{C\left(C N_{i}\right)^{|I-\tilde{I}|+|K-\tilde{K}|+p-\tilde{p}+q-\tilde{q}+\sigma\left|d_{1}+\gamma\right|}}{N_{i}^{s} N_{i}^{\left|d_{1}+\gamma\right|}} \\
\cdot\left\{\left\|T^{\tilde{q}} V^{\tilde{I}}\left(T^{\tilde{p}}\right)_{\left(\varphi_{i} \psi_{i}\right)^{(r+s)} V^{\tilde{K}} u \|}\right\|\right. \\
\left.+C^{N_{i}} \sup _{|\alpha+\beta| \leq \tilde{p}} N_{i}^{\tilde{q}+\tilde{p}+\left|\tilde{I}+K^{\prime}\right|-|\alpha+\beta|}\left\|\left(\varphi_{i} \psi_{i}\right)^{\left(r+s+\left|\tilde{K}-K^{\prime}\right|+|\alpha+\beta|\right)} u\right\|\right\} \\
\leq C \frac{\left(C N_{i}\right)^{|I-\tilde{I}|+|K-\tilde{K}|+p-\tilde{p}+q-\tilde{q}}}{N_{i}^{s}}\left\|T^{\tilde{q}} V^{\tilde{I}}\left(T^{\tilde{p}}\right)_{\left(\varphi_{i} \psi_{i}\right)^{(r+s)} V^{\tilde{K}} u \|} \sup _{\left|s^{\prime}\right| \leq 2 N_{i}}\right\|\left(\varphi_{i} \psi_{i}\right)^{\left(r+s^{\prime}\right)} u \| .
\end{aligned}
$$


Next, (5.3.32) or the third term in (5.3.19) is similarly bounded by

$$
\begin{aligned}
C^{N_{i}} \frac{N_{i}^{|I-\tilde{I}|+\left|K-K^{\prime}\right|+p+q-\tilde{q}+|\gamma|-s^{\prime}}}{N_{i}^{s} N_{i}^{\left|d_{1}+\gamma\right|}} N_{i}^{\left|d_{1}+\gamma\right|} \\
\cdot\left\{\left\|T^{\tilde{q}} V^{\tilde{I}}\left(\varphi_{i} \psi_{i}\right)^{(r+s)} V^{\tilde{K}} u\right\|+C^{N_{i}} N_{i}^{q+\left|\tilde{I}+K^{\prime}\right|}\left\|\left(\varphi_{i} \psi_{i}\right)^{\left(r+s+\left|\tilde{K}-K^{\prime}\right|\right)} u\right\|\right\} \\
\leq C^{N_{i}} \frac{N_{i}^{\left|I-I^{\prime}\right|+\left|K-K^{\prime}\right|+p+q-\tilde{q}}}{N_{i}^{s}}\left\|T^{\tilde{q}} V^{\tilde{I}}\left(\varphi_{i} \psi_{i}\right)^{(r+s)} V^{\tilde{K}} u\right\| \\
\quad+\frac{N_{i}^{|I|+|K|+p+q+r}}{N_{i}^{\left|s^{\prime}\right|}} \sup _{\left|s^{\prime}\right| \leq 2 N_{i}}\left\|\left(\varphi_{i} \psi_{i}\right)^{\left(r+s^{\prime}\right)} u\right\| .
\end{aligned}
$$

Thus (5.3.19) may be rewritten, using (5.3.11) and taking into account the bounds under the suprema in (5.3.31)- (5.3.34), as

Proposition 5.3.2. Let $|I|+p+q \leq N_{i},|K| \leq 2$; then

$$
\begin{aligned}
& \left\|V^{I} T^{q}\left[\left(T^{p}\right)_{\left(\varphi_{i} \psi_{i}\right)(r)}, \tilde{G}\right] V^{K} u\right\|
\end{aligned}
$$

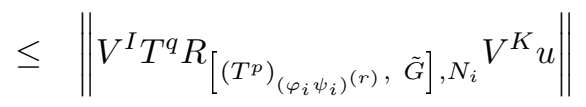

$$
\begin{aligned}
& +C \sup _{\substack{\Delta q+\Delta p+\Delta I+\Delta K \geq 1 \\
\Delta p>0}} C^{|\Delta|} N_{i}^{|\Delta|}\left\|V^{\tilde{I}} T^{\tilde{q}}\left(T^{\tilde{p}}\right)_{\left(\varphi_{i} \psi_{i}\right)^{(r+s)}} V^{\tilde{L}} u\right\| \\
& s \leq 2(\Delta I+\Delta+\Delta p+\Delta q) \\
& +C^{N_{i}} \sup _{\substack{\tilde{p}=0, \Delta q+p \geq 0 \\
\Delta I+\Delta L+\Delta q+\Delta p \geq 1 \\
s \leq N_{i}}} N_{i}^{|\Delta|}\left\|V^{\tilde{I}} T^{\tilde{q}}\left(\varphi_{i} \psi_{i}\right)^{(r+s)} V^{\tilde{L}} u\right\|,
\end{aligned}
$$

where $\Delta I=|I|-|\tilde{I}|, \Delta L=|K|-|\tilde{L}|, \Delta p=p-\tilde{p}, \Delta q=q-\tilde{q}, \Delta=\Delta I+\Delta L+$ $\Delta p+\Delta q,|\Delta|=|\Delta I|+|\Delta L|+|\Delta p|+|\Delta q|$.

We remark that $\tilde{L}$ may be large and hence $\Delta L$ may be negative.

We have

Proposition 5.3.3. We may take $\tilde{L}=0$ on the right hand side of (5.3.40).

Proof. The idea is to commute the $V$ fields behind $\left(T^{p}\right)_{\left(\varphi_{i} \psi_{i}\right)^{(r+s)}}$. We need only to be concerned with the $X$ vector fields; in fact the result of commuting back a $Y$ vector field will give a term analogous to the last in (5.2.8). These terms contain (positive order) derivatives of $\varphi_{i}^{\#}$ and will be estimated easily by exploiting the assumption on the $W F$ of $u$ in the leaves of the characteristic manifold.

As for the $X$ vector fields, it suffices to make the following two remarks. a) For a bounded number of $X^{\prime}$ 's we may commute them back to the left of $\left(T^{p}\right)_{\left(\varphi_{i} \psi_{i}\right)^{(r)}}$ by introducing a new constant and replacing $p$ by $p-\ell$ and $s$ by $s+\ell, \ell$ less or equal than the number of commuted fields. Doing so we do not alter anything else except the $C$ in $C^{N_{i}}$, since the second term in (4.2.8) (Proposition 4.2.5) is readily absorbed in the next to last term of (5.3.40).

b) For more $X$ 's (there are at least $\left|\beta_{3}^{\prime \prime}\right| \leq\left|\beta^{\prime \prime}\right| \leq p$ of them) we use Proposition 4.2.5 ensuring that only terms free of $\left(T^{p}\right)_{\left(\varphi_{i} \psi_{i}\right)(r)}, p>0$, occur when we commute 
$X^{\prime k}$ with $\left(T^{p}\right)_{\left(\varphi_{i} \psi_{i}\right)^{(r)}}$ and these terms are readily absorbed in the next to last term of (5.3.40).

Let us now go back to (5.3.19). We claim, for the remainder term, that

$$
\left\|V^{I} T^{q} R_{\left[{\left(T^{p}\right)}_{\left(\varphi_{i} \psi_{i}\right)(r)}, \tilde{G}\right], N_{i}} V^{K} u\right\| \leq\left(C K_{i}\right)^{N_{i}} N_{i}^{|I|+p+q+r},
$$

provided that $|I|+p+q \leq N_{i}, r \leq 2 N_{i}$.

Recall that $\sigma(\tilde{G})(x, \xi)=\Phi_{i}(x) g(x, \xi) \Psi_{i}(\xi)$, where $\Phi_{i}(x) \equiv 1$ in a neighborhood of $\bar{\Omega}$ and satisfies the inequalities $\left|D^{\alpha} \Phi_{i}(x)\right| \leq\left(C N_{I}\right)^{|\alpha|},|\alpha| \leq 3 N_{i}$. We have

Lemma 5.3.2. Let $a_{\alpha \beta}\left(\left(\varphi_{i} \psi_{i}\right)^{(s)}\right)=\sigma\left(B_{\alpha \beta}\left(\left(\varphi_{i} \psi_{i}\right)^{(s)}\right)\right)$. Then

$$
\begin{aligned}
& \left|\left(\frac{\partial}{\partial x}\right)^{\rho}\left(a_{\alpha \beta}\left(\left(\varphi_{i} \psi_{i}\right)^{(s)}\right)\right)_{(\gamma)}^{(\delta)}(x, \xi)\right| \\
& \quad \leq\left(C \tilde{K}_{i}\right)^{s+|\alpha+\beta+\rho+\gamma+\delta|} N_{i}^{s+|\alpha+\beta+\rho+\gamma|}\left(\frac{N_{i}}{|\xi|}\right)^{|\delta|} .
\end{aligned}
$$

Proof. We may write

$$
\begin{aligned}
& \left(\frac{\partial}{\partial x}\right)^{\rho}\left(a_{\alpha \beta}\left(\left(\varphi_{i} \psi_{i}\right)^{(s)}\right)\right)_{(\gamma)}^{(\delta)} \\
& =\sum_{r \leq|\alpha+\beta|,|\rho+\gamma|}\left(\frac{\partial}{\partial x}\right)^{r+\rho}\left(\frac{\partial}{\partial \xi}\right)^{\delta} a d_{X^{\prime}}^{\alpha} a d_{X^{\prime \prime}}^{\beta}\left(\left(\varphi_{i} \psi_{i}\right)^{(s)}\right) \\
& =\sum_{r \leq|\alpha+\beta|,|\rho+\gamma|} \frac{C^{|\alpha+\beta|} N_{i}^{r}}{\left(\frac{\partial}{\partial x}\right)^{r+\rho}\left(\frac{\partial}{\partial \xi}\right)^{\delta} x^{|\alpha+\beta|-r}\left(\left(\varphi_{i} \psi_{i}\right)^{(s+|\alpha+\beta|-r)}\right)} \\
& =\sum_{r \leq|\alpha+\beta|,|\rho+\gamma|} \underline{C^{|\alpha+\beta+\rho+\gamma|} N_{i}^{r}}\left(\frac{\partial}{\partial \xi}\right)^{\delta} x^{|\alpha+\beta|-r}\left(\left(\varphi_{i} \psi_{i}\right)^{(s+|\alpha+\beta+\rho+\gamma|-r)}\right),
\end{aligned}
$$

and the result follows from Proposition 4.3.8.

To estimate the remainder term, we write

$$
\begin{aligned}
\left.V^{I} T^{q} R_{\left[\left(T^{p}\right)_{\left(\varphi_{i} \psi_{i}\right)}(s)\right.}, \tilde{G}\right], N_{i} & V^{J} u \\
= & \sum_{r \leq|I|+p} \frac{C^{N_{i}} N_{i}^{r+r^{\prime}}}{\alpha ! \beta ! \gamma ! \delta !} x^{\leq|I|+P+q} D^{|I|+P+q-r} \\
& \times\left(F_{\alpha \beta, \gamma}^{\delta} H_{\alpha \beta, \delta}^{\gamma}-\left\{F_{\alpha \beta, \gamma}^{\delta} \circ H_{\alpha \beta, \delta}^{\gamma}\right\}_{N_{i}-|\delta+\gamma|}\right) D^{|J|-r^{\prime}} x^{|J|-r^{\prime}} u,
\end{aligned}
$$

where either

(i) $\quad F_{\alpha \beta, \gamma}^{\delta}=\tilde{G}_{(\delta)}^{(\gamma)}, H_{\alpha \beta, \delta}^{\gamma}=\left(B_{\alpha \beta}\left(\varphi_{i} \psi_{i}\right)^{(s)}\right)_{(\delta)}^{(\gamma)}$,
or
(ii) $\quad F_{\alpha \beta, \gamma}^{\delta}=\left(B_{\alpha \beta}\left(\varphi_{i} \psi_{i}\right)^{(s)}\right)_{(\delta)}^{(\gamma)}, H_{\alpha \beta, \delta}^{\gamma}=\tilde{G}_{(\delta)}^{(\gamma)}$,

with $\alpha=\beta=0$ in case (ii). In all cases $p+q+|I| \leq N_{i}, s \leq N_{i}$ and $|\gamma+\delta| \leq N_{i}$, $|\alpha+\beta| \leq p$.

The claim is proved by an application of Lemma A.1.

Summing up we have achieved the proof of the result of this section: 
Proposition 5.3.4. Assume that $u$ verifies the hypotheses of Theorem 2.1 (with $s=1$ for the sake of simplicity). Let $\tilde{G}\left(x, D_{x}\right)$ be an analytic pseudo-differential operator of degree $0,\left|I^{\prime}\right|+p+q \leq N_{i}, r \leq N_{i},|J| \leq 2$. Then there exists a positive constant $C=C(\tilde{G})$, independent of $N$, such that

$$
\begin{aligned}
& \left\|V^{I^{\prime}} T^{q}\left[\left(T^{p}\right)_{\left(\varphi_{i} \psi_{i}\right)^{(r)}}, \tilde{G}\right] V^{J} u\right\| \\
& \leq \quad C \sup _{\substack{\Delta \geq 0, \Delta p \geq 0, \Delta q \geq 0 \\
s \leq 2 \Delta,|\tilde{I}|-\left|I^{\prime}\right| \leq \Delta p}} \frac{C^{|\Delta|} N_{i}^{|\Delta|}}{N_{i}^{s}}\left\|V^{\tilde{I}} T^{\tilde{q}}\left(T^{\tilde{p}}\right)_{\left(\varphi_{i} \psi_{i}\right)^{(r+s)}} u\right\| \\
& \quad+C^{N_{I}} \sup _{\substack{\Delta q \geq 0, \Delta \geq 1 \\
s \leq N_{i}}} N_{i}^{\Delta-s}\left\|V^{\tilde{I}} T^{\tilde{q}}\left(\varphi_{i} \psi_{i}\right)^{(r+s)} u\right\| \\
& \quad+C C^{N_{i}} N_{i}^{\left|I^{\prime}\right|+p+q+r} K_{i}^{N_{i}+r}\|u\|,
\end{aligned}
$$

where $\Delta=\Delta I+\Delta p+\Delta q, \Delta I=\left|I^{\prime}\right|+|J|-|\tilde{I}|, \Delta p=p-\tilde{p}, \Delta q=q-\tilde{q}$, $|\Delta|=|\Delta I|+|\Delta q|+\Delta p$ and $V$ denotes, as usual in this section, a derivative either in the $X$ or $Y$ directions.

5.4. Reducing the order by half and the end of the proof of Theorem 2.1. Combining (5.2.10), Lemma 5.2.1 and Proposition 5.3.4 we obtain the estimate

$$
\begin{aligned}
& \sup _{\substack{I=I^{\prime}+J \\
|J| \leq 2}}\left\|Z^{I} T^{q}\left(T^{p}\right)_{\left(\varphi_{i} \psi_{i}\right)^{(r)}} u\right\|_{L^{2}(\Omega)} \\
& \leq C\left\{\left\|Z^{I^{\prime}} T^{q}\left(T^{p}\right)_{\left(\varphi_{i} \psi_{i}\right)}{ }^{(r)} \Phi_{i} P u\right\|_{L^{2}(\Omega)}\right. \\
& +\sup _{\substack{\Delta q \geq 0, \Delta p \geq 0 \\
\Delta \geq 1 \\
s \leq 2 \leq}} C^{|\Delta|} N_{i}^{\Delta-s}\left\|Z^{\tilde{I}} T^{\tilde{q}}\left(T^{\tilde{p}}\right)_{\left(\varphi_{i} \psi_{i}\right)^{(r+s)}} u\right\|_{L^{2}(\Omega)} \\
& +C^{N_{i}} \sup _{\substack{\Delta q \geq 0, \Delta p=p \\
s \leq N_{i}}} N_{i}^{\Delta-s}\left\|Z^{\tilde{I}} T^{\tilde{q}}\left(\varphi_{i} \psi_{i}\right)^{(r+s)} u\right\|_{L^{2}(\Omega)} \\
& +C^{N_{i}} N_{i}^{\left|I^{\prime}\right|+p+q+r} K_{i}^{N_{i}+r}\|u\|_{L^{2}(\Omega)} \\
& \left.+\underline{\left|I^{\prime}\right|} \sup _{\substack{\left|I^{\prime}\right| \\
q^{\prime \prime}=q+1}}\left\|Z^{I^{\prime}} T^{q^{\prime \prime}}\left(T^{p}\right)_{\left(\varphi_{i} \psi_{i}\right)^{(r)}} u\right\|_{L^{2}(\Omega)}\right\},
\end{aligned}
$$

where $Z$ denotes as usual either an $X$ - or a $Y$-derivative.

We point out explicitly that in deriving (5.4.1) we used the assumption that $\tilde{\varphi}_{i} \varphi_{i}^{\sharp(\alpha)} u$ is analytic, if $|\alpha|>0$, near our base point $\rho$.

Now starting with $i=q=r=I^{\prime}=0, p=p_{0}$ we use the estimate (5.4.1) with $i=0$ repeatedly. Each time we resubject the 2nd, 3rd and 5th terms in (5.4.1) to (5.4.1) to reduce $|I|+p+q$ (in the 3rd term $p=0$ and it cannot be subjected to (5.4.1) again). The aim is to obtain only the first four terms in the right hand side of (5.4.1), which will happen eventually. That is, we claim that the last term in (5.4.1) will eventually disappear. To see this, observe what happens after each iteration: in the last term $q$ has risen by 1 , but $|I|$ has dropped by 2 . After at most $\frac{N}{2}$ iterations, every term will either contain $P u$ or, we claim, have $p=0$ and have 
at most 1 free $Z$, with $q$ at most equal to $\frac{\left(p_{0}+2\right)}{2}$. To see that this is the case, note that in Proposition 5.3.4, $p$ decreases in each term on the right, while in (5.2.8), $p$ may keep it value. Now these terms arise only if $\left|I^{\prime}\right|+q>0$. Thus a given application of Proposition 5.3.4 may reduce $\left|I^{\prime}\right|$ or $p$ or $q$, but once $\left|I^{\prime}\right|+q$ reaches $0, p$ must decrease. The value of $q$ need not decrease, and may rise (via $[X, X]=T$ ) to one half the original value of $\left|I^{\prime}\right|+2+p$ or $\frac{p_{0}+2}{2}$ (new $Z^{\prime}$ 's arise in Proposition 5.3.4 - first term on the right hand side - only by a corresponding decrease of $p$ ). Thus we have proved

Proposition 5.4.1. For $p \leq N_{i}$,

$$
\begin{aligned}
\sup _{|J| \leq 2} & \left\|Z^{J}\left(T^{p}\right)_{\left(\varphi_{i} \psi_{i}\right)} u\right\|_{L^{2}(\Omega)} \\
\leq & C^{N_{i}}\left\{\sup _{\substack{s \leq N_{i}+2 \Delta\\
}} N_{i}^{\Delta-s}\left\|Z^{\tilde{I}} T^{\tilde{q}}\left(T^{\tilde{p}}\right)_{\left(\varphi_{i} \psi_{i}\right)^{(s)}} \Phi_{i} P u\right\|_{L^{2}(\Omega)}\right. \\
& +\sup _{\substack{s \leq 2 N_{i}, 2 \Delta \\
|J| \leq 2}} N_{i}^{\Delta-s}\left\|Z^{J}\left(\varphi_{i} \psi_{i}\right)^{(s)} T^{\tilde{q}} u\right\|_{L^{2}(\Omega)} \\
& \left.+N_{i}^{p-s} K_{i}^{N_{i}}\|u\|_{L^{2}(\Omega)}\right\}
\end{aligned}
$$

where $K_{i}$ satisfies the bounds (3.1.1) - (3.1.8) and $\Delta=p-\tilde{p}+q+\tilde{q}-|\tilde{I}|$.

Lemma 5.4.1. For $|I|+p+q \leq N_{i}, s \leq 2 N_{i}$

$$
\frac{1}{N_{i}^{s}}\left\|X^{I} T^{q}\left(T^{p}\right)_{\left(\varphi_{i} \psi_{i}\right)^{(s)}} \Phi_{i} P u\right\|_{L^{2}(\Omega)} \leq C_{f}^{N_{i}} K_{i}^{N_{i}} N_{i}^{|I|+p+q} .
$$

Proof. The proof is clear due to the assumptions of Theorem 2.1 and by Proposition 4.3.2.

For the second term on the right in Proposition 5.4.1 we pass to a new pair of localizing functions, $\varphi_{i^{\prime}}, \psi_{i^{\prime}}$, where $i^{\prime}$ is the largest integer such that $q+2 \leq N_{i^{\prime}}$ (so that $\left.\tilde{q} !^{-1}\right) \leq C^{N_{i}} N_{i}^{-\tilde{q}}$.

Proposition 5.4.2. Let $|J| \leq 2$ and $s \leq 2 N_{i}, \tilde{q} \leq N_{i}$; then

$$
\frac{1}{N_{i}^{s}}\left\|Z^{J}\left(\varphi_{i} \psi_{i}\right)^{(s)}\left(T^{\tilde{q}}-\left(T^{\tilde{q}}\right)_{\varphi_{i}^{\prime} \psi_{i}^{\prime}}\right) u\right\|_{L^{2}(\Omega)} \leq C^{N_{i}+1} N_{i}^{\tilde{q}}
$$

Proof. This proposition is proved by arguing exactly as in the proof of Proposition 4.3.1.

Proposition 5.4.3. For $S \leq 2 N_{i}$,

$$
\begin{aligned}
& \sup _{|J| \leq 2}\left\|Z^{J}\left(\varphi_{i} \psi_{i}\right)^{(s)}\left(T^{\tilde{q}}\right)_{\varphi_{i}^{\prime} \psi_{i}^{\prime}} u\right\|_{L^{2}(\Omega)} / N_{i}^{s} \\
& \quad \leq C^{N_{i}} K_{i}^{N_{i}} \sup _{|J| \leq 2}\left\|Z^{J}\left(T^{\tilde{q}}\right)_{\varphi_{i}^{\prime} \psi_{i}^{\prime}} u\right\|_{L^{2}(\Omega)} .
\end{aligned}
$$

Proof. This proof is a consequence of the bounds (3.1.1) - (3.1.8) and the bounds on $\left(\varphi_{i} \psi_{i}\right)^{(s)}$ (Proposition 4.3.2).

Thus we have shown: 


\section{Proposition 5.4.4.}

$$
\begin{aligned}
\sup _{\substack{\mid J \backslash \leq 2 \\
p \leq N_{i}}} & \left\|Z^{I}\left(T^{p}\right)_{\varphi_{i} \psi_{i}} u\right\|_{L^{2}(\Omega)} \\
\leq & C^{N_{i}} K_{i}^{N_{i}}\left\{N_{i}^{p}\|u\|_{L^{2}(\Omega)}+\sup _{\substack{p_{1} \leq\left(N_{i}+2\right) / 2 \\
|J| \leq 2}} N_{i}^{p-p_{1}}\left\|Z^{J}\left(T^{p_{1}}\right)_{\varphi_{i}^{\prime} \psi_{i}^{\prime}} u\right\|_{L^{2}(\Omega)}\right\} \\
& +C_{f}^{N_{i}} N_{i}^{p} K_{i}^{N_{i}} .
\end{aligned}
$$

Iterating $\log _{2} N$ times (5.4.4), starting with $i=0, p \leq N$, we obtain

$$
\begin{aligned}
& \sup _{\substack{p \leq N \\
|J| \leq 2}}\left\|Z^{J}\left(T^{p}\right)_{\varphi_{0} \psi_{0}} u\right\|_{L^{2}(\Omega)} \\
& \quad \leq C^{N}\left(\Pi K_{i}^{N_{i}}\right) N^{N}\left\{\|u\|_{L^{2}(\Omega)}+C_{f}^{N}\right\} \\
& \quad \leq C^{N} N !,
\end{aligned}
$$

thus proving Theorem 2.1 .

\section{Proof of Theorem 2.2}

To prove Theorem 2.2 we prove a slightly more general result.

We denote by $Q$ a pseudo-differential operator of order zero whose symbol has small conic support (soo to be used to microlocalize), and by $D$ any of the partial derivatives $\frac{\partial}{\partial x_{j}}, j \leq n$. Our starting point in terms of estimates is

$$
\sum_{j=1}^{m}\left\|X_{j} v\right\|_{L^{2}}^{2}+\|v\|_{\delta}^{2} \leq C\left\{\operatorname{Re}(P v, v)+\|v\|_{L^{2}}^{2}\right\},
$$

where $\delta$ is a real number, $0<\delta \leq 1$ and the operator $P$ has the form

$$
P=\sum_{|I| \leq 2} a_{I} X_{I}+i b X_{0} .
$$

Theorem 6.1. If $\left(x_{0}, \xi_{0}\right) \notin W F_{s}(f=P u)$ for some distribution $u$, and some $s \geq 1 / \delta$, then $\left(x_{0}, \xi_{0}\right) \notin W F_{s}(u)$.

Proof. Assuming the solution $u$ belongs to $C^{\infty}$, we need to obtain reasonable estimates for $D^{\alpha} Q u$ of the form

$$
\left|D^{\alpha} Q u\right| \leq C^{|\alpha|+1} \alpha !^{s}
$$

or, what amounts to the same thing,

$$
\left\|D^{\alpha} Q u\right\|_{L^{2}} \leq C^{|\alpha|+1} N^{s|\alpha|}, \quad|\alpha| \leq N .
$$

Now in view of the maximality and subellipticity of $P$, we shall profit from all aspects of the a priori estimate and apply it to $v=D^{\alpha} Q u$ :

$$
\begin{gathered}
\sum_{1}^{m}\left\|X_{j} D^{\alpha} Q u\right\|_{L^{2}}^{2}+\left\|D^{\alpha} Q u\right\|_{\delta}^{2} \\
\leq C\left\{\operatorname{Re}\left(P D^{\alpha} Q u, D^{\alpha} Q u\right)_{L^{2}}+\left\|D^{\alpha} Q u\right\|_{L^{2}}^{2}\right\} \\
\leq C\left\{\left\|D^{\alpha} Q P u\right\|_{L^{2}}^{2}+\left\|D^{\alpha} Q u\right\|_{L^{2}}^{2}+\operatorname{Re}\left(\left[P, D^{\alpha} Q\right] u, D^{\alpha} Q u\right)_{L^{2}}\right\}
\end{gathered}
$$


The essential work is to commute $P$ with $D^{\alpha} Q$, and we write this as follows. Schematically writing $P=\left(a X_{j}\right)\left(a X_{k}\right)$ (the first order term with $i X_{0}$ will not pose a problem), we write

$$
\begin{gathered}
\operatorname{Re}\left(\left[P, D^{\alpha} Q\right] u, D^{\alpha} Q u\right)_{L^{2}}=\left(\operatorname{aXad}_{a X}\left(D^{\alpha} Q\right) u, D^{\alpha} Q u\right)_{L^{2}} \\
+\left(a d_{a X}^{2}\left(D^{\alpha} Q\right) u, D^{\alpha} Q u\right)_{L^{2}} \\
=E_{1}+E_{2} .
\end{gathered}
$$

If we write

$$
S^{(\gamma)}=a d_{a X}^{\gamma}(S)
$$

for any pseudo-differential operator $S, E_{1}$ then consists of terms with an $X$ free, which may be integrated by parts (modulo zero order terms). A weighted Schwarz inequality is used on such terms, with the right hand member being absorbed on the left hand side of the inequality. Thus:

$$
\left|E_{1}\right| \leq \frac{1}{2} \sum_{j=1}^{m}\left\|X_{j} D^{\alpha} Q u\right\|_{L^{2}}^{2}+C\left\|\Lambda^{-|\delta|}\left(D^{\alpha} Q\right)^{(1)} u\right\|_{\delta}^{2} .
$$

In much the same way, $E_{2}$ may be estimated

$$
\left|E_{2}\right| \leq \frac{1}{2}\left\|D^{\alpha} Q u\right\|_{\delta}^{2}+C\left\|\Lambda^{-2|\delta|}\left(D^{\alpha} Q\right)^{(2)} u\right\|_{\delta}^{2} .
$$

Thus using (6.2), using the above estimates for the errors $E_{1}$ and $E_{2}$, and iterating $|\alpha| /|\delta|$ times we have:

$$
\begin{aligned}
& \sum_{j=1}^{m}\left\|X_{j} D^{\alpha} Q u\right\|_{L^{2}}^{2}+\left\|D^{\alpha} Q u\right\|_{\delta}^{2} \\
& \leq C^{|\alpha|}\left\{\sup _{\substack{\sum j_{k} \leq|\alpha| /|\delta| \\
1 \leq j_{k} \leq 2}}\left\|\Pi_{k}\left(\Lambda^{-|\delta| j_{k}}\left(D^{\alpha} Q\right)^{\left(j_{k}\right)}\right) P u\right\|_{L^{2}}^{2}\right. \\
& \left.+\sup _{\frac{|\alpha|}{|\delta| \leq \sum j_{k} \leq \frac{|\alpha|}{\delta}+1} 1 \leq j_{k} \leq 2}\left\|\Pi_{k}\left(\Lambda^{-|\delta| j_{k}}\left(D^{\alpha} Q\right)^{\left(j_{k}\right)}\right) u\right\|_{\delta}^{2}\right\} .
\end{aligned}
$$

What has happened, as can already be seen in the first step, is that for every derivative that lands on $D^{\alpha} Q$ as a bracket, there is a "gain" of $|\delta|$ derivatives. Thus either multiple iterations with the same $Q$ are required or one could perhaps replace the $Q$ with another $Q$ after each such gain, although this does not seem to be any simpler. In any case, estimating the behavior, in $L^{2}$ or in $H^{|\delta|}$ of these very high order commutators of pseudo-differential operators is very far from simple. However we have already carried out an extremely precise analysis of each such bracket in (5.3.11) and what follows (5.3.11). While that analysis is for the carefully balanced operators required to localize $T^{p}$, the present situation requires no such balance and is thus essentially simpler. We do not carry out the full details since we would be repeating much of the analysis following (5.3.11). 


\section{Appendix}

In this Appendix we gather some general-purpose results, mostly well known, which are used and referenced throughout the paper. Sometimes we shall prove statements a bit more general than needed at a specific point.

Lemma A.1. Let $F\left(x, D_{x}\right) \in L^{m}\left(\mathbb{R}_{x}^{n}\right), H\left(x, D_{x}\right) \in L^{m^{\prime}}\left(\mathbb{R}_{x}^{n}\right)$ be pseudo-differential operators with (full) symbols $f(x, \xi), h(x, \xi)$ respectively. Denote by $\hat{h}(\eta, \xi)=$ $\int e^{-i x \cdot \eta} h(x, \xi) d x$ the Fourier transform of $h$ with respect to $x$. Then for any multiindices $a, b \in \mathbb{Z}_{+}^{n}, \forall M \in \mathbb{Z}_{+}, \forall w \in C_{0}^{\infty}\left(\mathbb{R}^{n}\right)$ we have

$$
\begin{gathered}
D_{x}^{a}\left(F\left(x, D_{x}\right) \circ H\left(x, D_{x}\right)-\{F \circ H\}_{M}\left(x, D_{x}\right)\right) D_{x}^{b} w \\
=\underline{C^{|a+b|+n}} \int e^{i x \cdot \xi}\left(\int r_{c_{1}}(x, \eta, \xi) d \eta\right) \hat{w}(\xi) d \xi,
\end{gathered}
$$

where

$$
\begin{aligned}
r_{c_{1}}(x, \eta, \xi)= & \sum_{|\varepsilon|=M} \frac{(1+|\eta|)^{-n-1}}{\varepsilon !}\left(h^{\left(\varepsilon+b_{2}+c_{1}\right)}\right) \wedge e^{i x \cdot \eta} \\
& \cdot\left(\int_{0}^{1} f_{(\varepsilon)}^{\left(a_{2}\right)}(x, \xi+\rho \eta)(\xi+\rho \eta)^{a_{1}+b_{1}-c_{1}}(1-\rho)^{\left|c_{1}+\varepsilon\right|} d \rho\right) ;
\end{aligned}
$$

and where

$$
\{F \circ H\}_{M}(x, \xi)=\sum_{|\alpha|<M} \frac{1}{\alpha !} f_{(\alpha)}(x, \xi) h^{(\alpha)}(x, \xi),
$$

$f_{(\alpha)}^{(\beta)}(x, \xi)=\partial_{\xi}^{\alpha} D_{x}^{\beta} f(x, \xi)$ and $\varepsilon, a_{1}, a_{2}, b_{1}, b_{2}, c_{1} \in \mathbb{Z}_{+}^{n}$ are multi-indices such that

$$
\left|b_{1}+b_{2}\right|=|b|+n+1 \quad \text { and } \quad c_{1} \leq a_{1}+b_{1} .
$$

Proof. By a direct calculation:

$$
\begin{aligned}
& D_{x}^{a}\left(F\left(x, D_{x}\right) \circ H\left(x, D_{x}\right)-\{F \circ H\}_{M}\left(x, D_{x}\right)\right) D_{x}^{b} w \\
&=D_{x}^{a} \iint e^{i x \cdot(\xi+\eta)}\left[f(x, \xi+\eta)-\sum_{|\varepsilon|<M} f_{(\varepsilon)}(x, \xi) \frac{\eta^{\varepsilon}}{\varepsilon !}\right] \hat{h}(\eta, \xi) \xi^{b} \hat{w}(\xi) d \eta d \xi \\
&=C^{|a+b|+n} \iint e^{i x \cdot(\xi+\eta)}(\xi+\eta)^{a_{1}+b_{1}} \sum_{|\varepsilon|=M} \frac{M}{\varepsilon !}(1+|\eta|)^{-n-1} \\
& \cdot \int_{0}^{1} f_{(\varepsilon)}^{\left(a_{2}\right)}(x, \xi+\rho \eta)(1-\rho)^{|\varepsilon|} d \rho\left(h^{\left(\varepsilon+b_{2}\right)}\right) \hat{n}(\eta, \xi) \hat{w}(\xi) d \eta d \xi,
\end{aligned}
$$

where $a_{1}+a_{2}=a,\left|b_{1}+b_{2}\right| \leq b+n+1$. The latter can be written as a sum (over $c_{1} \leq a_{1}+b_{1}$ ) of expressions of the form

$$
\underline{C^{|a+b|+n}} \int e^{i x \cdot \xi}\left(\int r_{c_{1}}(x, \eta, \xi) d \eta\right) \hat{w}(\xi) d \xi
$$

where

$$
\begin{aligned}
r_{c_{1}}(x, \eta, \xi)= & \sum_{|\varepsilon|=M} \frac{1}{\varepsilon !}(1+|\eta|)^{-n-1}\left(h^{\left(\varepsilon+b_{2}+c_{1}\right)}\right) \wedge(\eta, \xi) e^{i x \cdot \eta} \\
& \cdot\left(\int_{0}^{1} f_{(\varepsilon)}^{\left(a_{2}\right)}(x, \xi+\rho \eta)(\xi+\rho \eta)^{a_{1}+b_{1}-c_{1}}(1-\rho)^{\left|c_{1}+\varepsilon\right|} d \rho\right) .
\end{aligned}
$$


Here we wrote $\xi+\eta=\xi+\rho \eta+(1-\rho) \eta$, and this completes the proof of the lemma.

Lemma A.2. Let $\alpha, \beta \in \mathbb{Z}_{+}^{n}$ be multi-indices. Then

$$
\begin{gathered}
\partial_{x}^{\alpha} x^{\beta}=\sum_{\delta \leq \alpha, \beta}\left(\begin{array}{l}
\alpha \\
\delta
\end{array}\right)\left(\begin{array}{l}
\beta \\
\delta
\end{array}\right) \delta ! x^{\beta-\delta} \partial_{x}^{\alpha-\delta} ; \\
x^{\alpha} \partial_{x}^{\beta}=\sum_{\delta \leq \alpha, \beta}(-1)^{|\delta|}\left(\begin{array}{l}
\alpha \\
\delta
\end{array}\right)\left(\begin{array}{l}
\beta \\
\delta
\end{array}\right) \delta ! \partial_{x}^{\beta-\delta} x^{\alpha-\delta} .
\end{gathered}
$$

Proof. (A.3) is proved by Leibniz' formula:

$$
\partial_{x}^{\alpha} x^{\beta} u=\sum_{\delta \leq \alpha, \beta}\left(\begin{array}{l}
\alpha \\
\delta
\end{array}\right) \beta(\beta-1) \cdots(\beta-\delta+1) x^{\beta-\delta} \partial_{x}^{\alpha-\delta} u,
$$

whereas (A.4) can be proved taking the Fourier transform of (A.3).

Lemma A.3. Let $\alpha_{j}, \beta_{j} \in \mathbb{Z}_{+}^{n}$ be multi-indices, $j=1, \ldots, r$. Denoting by $\alpha=$ $\sum_{i=1}^{r} \alpha_{i}, \beta=\sum_{j=1}^{r} \beta_{j}$, we have that

$$
x^{\alpha_{1}} \partial_{x}^{\beta_{1}} x^{\alpha_{2}} \partial_{x}^{\beta_{2}} \cdots x^{\alpha_{r}} \partial_{x}^{\beta_{r}}=\sum_{\delta \leq \alpha, \beta} \underline{C^{|\beta|}} \underline{|\alpha|^{|\delta|}} x^{\alpha-\delta} \partial_{x}^{\beta-\delta} .
$$

Proof. Using (A.3) we want to move the last group of $x$ 's in (A.5), i.e. $x^{\alpha_{r}}$, to the left. This yields:

$$
\begin{aligned}
& x^{\alpha_{1}} \partial_{x}^{\beta_{1}} x^{\alpha_{2}} \partial_{x}^{\beta_{2}} \cdots x^{\alpha_{r}} \partial_{x}^{\beta_{r}} \\
& \quad=\sum_{\substack{\delta_{r} \leq \alpha_{r} \\
\delta_{r} \leq \beta_{r-1}}}\left(\begin{array}{c}
\beta_{r-1} \\
\delta_{r}
\end{array}\right) \underline{\left|\alpha_{r}\right| \delta_{r} \mid} x^{\alpha_{1}} \partial_{x}^{\beta_{1}} \cdots \partial_{x}^{\beta_{r-2}} x^{\alpha_{r-1}+\alpha_{r}-\delta_{r}} \partial_{x}^{\beta_{r-1}+\beta_{r}-\delta_{r}} .
\end{aligned}
$$

Iterating this procedure, i.e. moving $x^{\alpha_{r-1}+\alpha_{r}-\delta_{r}}$ to the left and using again (A.3), we obtain

$$
\begin{aligned}
& x^{\alpha_{1}} \partial_{x}^{\beta_{1}} x^{\alpha_{2}} \partial_{x}^{\beta_{2}} \cdots x^{\alpha_{r}} \partial_{x}^{\beta_{r}} \\
& =\quad \sum_{\delta_{r} \leq \alpha_{r}, \beta_{r-1}} \quad\left(\begin{array}{c}
\beta_{r-1} \\
\delta_{r}
\end{array}\right)\left(\begin{array}{c}
\beta_{r-2} \\
\delta_{r-1}
\end{array}\right) \underline{\left|\alpha_{r}\right|^{\left|\delta_{r}\right|}} \underline{\left|\alpha_{r-1}+\alpha_{r}-\delta_{r}\right|^{\left|\delta_{r-1}\right|}} \\
& \begin{array}{c}
\delta_{r} \leq \alpha_{r}, \beta_{r-1} \\
\delta_{r-1} \leq \alpha_{r}+\alpha_{r-1}-\delta_{r}, \beta_{r-2}
\end{array} \\
& \cdot x^{\alpha_{1}} \partial_{x}^{\beta_{1}} \cdots \partial_{x}^{\beta_{r-3}} x^{\alpha_{r}+\alpha_{r-1}+\alpha_{r-2}-\delta_{r}-\delta_{r-1}} \partial_{x}^{\beta_{r-2}+\beta_{r-1}+\beta_{r}-\delta_{r}-\delta_{r-1}} \\
& =\cdots \\
& =\sum_{j=0}^{r} \sum_{\substack{\delta_{r-j} \leq \beta_{r-j-1} \\
\delta_{r-j} \leq \sum_{\ell=0}^{j}\left(\alpha_{r-\ell}-\delta_{r-\ell-1}\right)}} \prod_{s=1}^{r}\left(\begin{array}{c}
\beta_{r-s} \\
\delta_{r-s+1}
\end{array}\right) \underline{|\alpha|^{|\delta|}} x^{\alpha-\sum_{i=1}^{r} \delta_{i}} \partial_{x}^{\beta-\sum_{i=0}^{r} \delta_{i}},
\end{aligned}
$$

and this proves the assertion since $\prod_{s=1}^{r}\left(\begin{array}{c}\beta_{r-s} \\ \delta_{r-s+1}\end{array}\right)=\underline{C^{|\beta|}}$.

Proposition A.1. If $X^{J}$ means $X_{1}^{j_{1}} \cdots X_{2 k}^{j_{2 k}}$, where the $X$ 's are the same vector fields of Section 4.1, then

$$
X^{J}=\underline{C^{|J|}} \sum_{J^{\prime} \leq J}|J|^{\left|J^{\prime}\right|} x^{J-J^{\prime}} \partial_{x}^{J-J^{\prime}}
$$

Proof. This is an application of Lemma A.3. 


\section{REFERENCES}

[1] K.G. Andersson, Propagation of Analyticity of Solutions of Partial Differential Equations with Constant Coefficients. Ark. för Matematik, 8 (1970), 277-302. MR 45:8986

[2] P. Bolley, J. Camus and L. Rodino, Hypoellipticité Analytique-Gevrey et Itérés d'opérateurs. Rend. Sem. Mat. Univ. Politecn. Torino, 45 (1987) 1-61. MR 91f:35073

[3] L. Boutet de Monvel, A. Grigis and B. Helffer, Parametrixes d'opérateurs pseudodifférentiels à caractéristiques multiples. Astérisque, 34-35 (1976) 93-121. MR 58:12046

[4] L. Boutet de Monvel and P. Krée, Pseudodifferential Operators and Gevrey Classes. Ann. Inst. Fourier Grenoble, 17 (1967), 295-323. MR 37:1760

[5] L. Hörmander, Uniqueness Theorems and Wave Front Sets for Solution of Linear Differential Equations with Analytic Coefficients. Comm. Pure Appl. Math., 24 (1971), 671-704. MR 45:3917

[6] L. Hörmander, A Class of Hypoelliptic Pseudo-differential Operators with Double Characteristics. Math. Ann., 217 (1975) 165-188. MR 51:13774

[7] L. Hörmander, The Analysis of Linear Partial Differential Operators I - IV. Springer-Verlag, Berlin, 1983-85. MR 85g:35002a, MR 85g:35002b, MR 87d:35002a, MR 87d:35002b

[8] K. Kajitani and S. Wakabayashi, Hypoelliptic Operators in Gevrey Classes. in Recent Developments in Hyperbolic Equations, L. Cattabriga et al., Eds., Pitman Research Notes in Math. n. 183 (1988), 115-134. MR 91g:35184

[9] G. Métivier, Une Classe d'Opérateurs non Hypoelliptiques Analytiques. Indiana Univ. Math. J., 29 (1980), 823-860. MR 82a:35029

[10] G. Métivier, Analytic Hypoellipticity for Operators with Multiple Characteristics. Comm. in P. D. E., 6 No.1 (1981), 1-90. MR 82g:35030

[11] G. Métivier, Non Hypoellipticité Analytique pour $D_{x}^{2}+\left(x^{2}+y^{2}\right) D_{y}^{2}$. Comptes Rendus Acad. Sci. Paris, 292 (1981), 401-404. MR 82b:35039

[12] G. Métivier, Non Hypoellipticité Analytique pour des Opérateurs à Caracteristiques Doubles. Séminaire Goulaouic-Meyer-Schwartz, École Polytechmique, 1981 - 1982, Exposé XII, 1-12. MR 84c:35027

[13] J. Sjöstrand, Analytic Wavefront Sets and Operators with Multiple Characteristics. Hokkaido Math. J., 12 (1983), 392-433. MR 85e:35022

[14] D. Tartakoff, On the Local Real Analyticity of Solutions to $\square_{b}$ and the $\bar{\partial}-$ Neumann Problem. Acta Math., 145 (1980), 117-204. MR 81k:35033

[15] D. Tartakoff, Local Analytic Hypoellipticity for $\square_{b}$ on non degenerate Cauchy Riemann Manifolds. Proc. Nat. Acad. Sci. U.S.A., 75(7) (1978), 3027-3028. MR 80g:58045

[16] F. Trèves, Analytic Hypoellipticity of a Class of Pseudo-differential Operators with Double Characteristics. Comm. in P. D. E., 3 No.1 (1978) 475-642. MR 58:11867

Dipartimento di Matematica, Università di Bologna, 40127 Bologna, Italy

E-mail address: bove@dm.unibo.it

Department of Mathematics, University of Illinois at Chicago, 851 S. Morgan, m/C 249, Chicago Illinois 60607-7045, U.S.A.

E-mail address: dst@uic.edu 